\title{
Existence of a stable polarized vacuum in the Bogoliubov-Dirac-Fock approximation
}

\author{
Christian HAINZL, Mathieu LEWIN \& Éric SÉRÉ \\ CEREMADE, UMR CNRS 7534, Université Paris IX Dauphine, Place du \\ Maréchal De Lattre De Tassigny, 75775 Paris, Cedex 16, France. \\ E-mail: hainzl, lewin, sere@ceremade.dauphine.fr
}

\begin{abstract}
According to Dirac's ideas, the vacuum consists of infinitely many virtual electrons which completely fill up the negative part of the spectrum of the free Dirac operator $D^{0}$. In the presence of an external field, these virtual particles react and the vacuum becomes polarized.

In this paper, following Chaix and Iracane (J. Phys. B, 22, 3791-3814, 1989), we consider the Bogoliubov-Dirac-Fock model, which is derived from no-photon QED. The corresponding BDF-energy takes the polarization of the vacuum into account and is bounded from below. A BDF-stable vacuum is defined to be a minimizer of this energy. If it exists, such a minimizer is solution of a self-consistent equation.

We show the existence of a unique minimizer of the BDF-energy in the presence of an external electrostatic field, by means of a fixed-point approach. This minimizer is interpreted as the polarized vacuum.
\end{abstract}

\section{Introduction}

The relativistic quantum theory of electrons and positrons is based on the free Dirac operator, which is defined by

$$
D^{0}=-i \sum_{k=1}^{3} \alpha_{k} \partial_{k}+\beta:=-i \boldsymbol{\alpha} \cdot \nabla+\beta
$$

where $\boldsymbol{\alpha}=\left(\alpha_{1}, \alpha_{2}, \alpha_{3}\right)$ and

$$
\beta=\left(\begin{array}{cc}
I_{2} & 0 \\
0 & -I_{2}
\end{array}\right), \quad \alpha_{k}=\left(\begin{array}{cc}
0 & \sigma_{k} \\
\sigma_{k} & 0
\end{array}\right)
$$

with

$$
\sigma_{1}=\left(\begin{array}{cc}
0 & 1 \\
1 & 0
\end{array}\right), \quad \sigma_{2}=\left(\begin{array}{cc}
0 & -i \\
i & 0
\end{array}\right), \quad \sigma_{3}=\left(\begin{array}{cc}
1 & 0 \\
0 & -1
\end{array}\right) .
$$

*Second address: Laboratoire de Mathématiques Paris-Sud. Bât. 425, 91405 Orsay Cedex. France 
We follow here mainly the notation of Thaller's book [54. We have chosen a system of units such that $\hbar=c=1$, and also such that the mass $m_{e}$ of the electron is normalized to 1 .

The operator $D^{0}$ acts on 4 -spinors, i.e. functions $\psi \in \mathcal{H}:=L^{2}\left(\mathbb{R}^{3}, \mathbb{C}^{4}\right)$. It is self-adjoint on $\mathcal{H}$, with domain $H^{1}\left(\mathbb{R}^{3}, \mathbb{C}^{4}\right)$ and form domain $H^{1 / 2}\left(\mathbb{R}^{3}, \mathbb{C}^{4}\right)$. Moreover, it is defined to ensure

$$
\left(D^{0}\right)^{2}=-\Delta+1
$$

The spectrum of $D^{0}$ is $(-\infty ;-1] \cup[1 ; \infty)$. In what follows, the projector associated with the negative part of the spectrum of $D^{0}$ will be denoted by $P^{0}$ :

$$
P^{0}:=\chi_{(-\infty ; 0)}\left(D^{0}\right)
$$

We then have

$$
\begin{gathered}
D^{0} P^{0}=P^{0} D^{0}=-\sqrt{1-\Delta} P^{0}=-P^{0} \sqrt{1-\Delta} \\
D^{0}\left(1-P^{0}\right)=\left(1-P^{0}\right) D^{0}=\sqrt{1-\Delta}\left(1-P^{0}\right)=\left(1-P^{0}\right) \sqrt{1-\Delta}
\end{gathered}
$$

and

$$
\mathcal{H}=\mathcal{H}_{-}^{0} \oplus \mathcal{H}_{+}^{0},
$$

where $\mathcal{H}_{-}^{0}:=P^{0} \mathcal{H}$ and $\mathcal{H}_{+}^{0}:=\left(1-P^{0}\right) \mathcal{H}$.

The fact that the spectrum of $D^{0}$ is not bounded from below is the source of many difficulties in Relativistic Quantum Mechanics. To explain why a free electron does not dissolve into the lower continuum, Dirac's idea [13, 14] was to postulate that in the absence of external field, the vacuum contains infinitely many virtual electrons which completely fill up the negative part of the spectrum of $D^{0}$. This Dirac Sea should be seen as an infinite Slater determinant $\Omega^{0}=\psi_{1}^{0} \wedge \cdots \wedge \psi_{i}^{0} \wedge \cdots$ where $\left(\psi_{i}^{0}\right)_{i \geq 1}$ is an orthonormal basis of $\mathcal{H}_{-}^{0}$, whose density matrix is precisely

$$
P^{0}=\sum_{i \geq 1}\left|\psi_{i}^{0}\right\rangle\left\langle\psi_{i}^{0}\right|
$$

The projector $P^{0}$ is often called the bare vacuum [9].

Let us now add an external coulomb field, created for instance by a system of smeared nuclei. The density of protons in this system is a nonnegative function ${ }^{1}$ $n$ such that $\int_{\mathbb{R}^{3}} n=Z$, the total number of protons in the nuclei. In our system of units, the external coulomb potential felt by the electrons is $-\alpha \varphi$, where $\varphi=n * \frac{1}{\frac{1}{1}}$ and $\alpha$ is a small dimensionless coupling constant, usually called the Sommerfeld fine structure constant. The Dirac operator with this external field is

$$
D^{\alpha \varphi}:=D^{0}-\alpha \varphi .
$$

Dirac postulated that the charge of the bare vacuum is not measurable. Indeed, $P^{0}$ commutes with translations, so its density of charge must be constant,

\footnotetext{
${ }^{1}$ However, we shall not limit to nonnegative $L^{1}$ densities $n$ in this paper, since the model we want to study is able to describe the vacuum interacting with both matter and antimatter.
} 
and cannot create any electric force. However, in the presence of an external field, the virtual electrons react, by occupying the negative energy states of a new Dirac operator which does not commute with translations: the vacuum is polarized. This polarization of the dressed vacuum, which takes the form of a local density of charge, is measurable in practice.

The vacuum polarization plays a minor role in the calculation of the Lamb shift for the ordinary hydrogen atom (comparing to other electrodynamic phenomena), but it is important for high- $Z$ atoms 43. and even plays a crucial role in muonic atoms [18, 24]. It also explains the production of electron-positron pairs, observed experimentally in heavy ions collisions [1, 46, 37, 50, 20.

When the external field is not too strong, a good approximation (called the Furry picture [21] ) is to define the polarized vacuum as the projector

$$
P^{\alpha \varphi}:=\chi_{(-\infty ; 0)}\left(D^{\alpha \varphi}\right)
$$

Note that in reality, the polarized vacuum modifies the electrostatic field, and the virtual electrons react to the corrected field. This remark naturally leads to a self-consistent equation for the dressed vacuum, and to a fixed-point iterative procedure for solving it. If one starts the procedure from $P^{0}$, the first iteration gives $P^{\alpha \varphi}$, and this explains why the Furry picture is a good approximation. But in practice, corrections to the Furry picture are necessary for high accuracy computations of electronic levels near heavy nuclei. These corrections can be interpreted as the second iteration in a Banach fixed-point algorithm (see, e.g., [43, section 8.2]).

In physics, self-consistent equations are usually derived as Euler-Lagrange equations of an energy functional. It is the case, for instance, in the nonrelativistic Hartree-Fock model [40]. Similarly, the self-consistent equation for the vacuum has a variational interpretation: it is satisfied by a minimizer of the Bogoliubov-Dirac-Fock (BDF) energy functional. This functional was first introduced by Chaix and Iracane [9], as a possible cure to the fundamental problems associated with standard relativistic quantum chemistry calculations.

In these calculations, electrons near heavy nuclei are usually treated, in first approximation, by the Dirac-Fock model [53], a variant of Hartree-Fock in which the kinetic energy operator $-\Delta / 2$ is replaced by the free Dirac operator $D^{0}$. This approach gives results that are in excellent agreement with experimental data [34, 26, 12, 41. When a higher accuracy is needed, the more sophisticated multiconfiguration Dirac-Fock-model is used to take into account correlation effects [25], and one can even compute the small corrections predicted by QED (vacuum polarization and radiative corrections), using perturbation methods. However, the Dirac-Fock model suffers from an important defect: the corresponding energy is not bounded from below, contrary to the nonrelativistic Hartree-Fock case, and this leads to important computational difficulties (see [9] for a discussion and detailed references). From the mathematical viewpoint, one can prove that the Dirac-Fock functional has critical points which are solutions of the Dirac-Fock equations [15, 44, but these critical points have an infinite Morse index, and the rigorous definition of a ground state is delicate [16. 17. 
The second problem with Dirac-Fock is its physical derivation: one would like to interpret this model as a variational approximation of Quantum Electrodynamics (QED), which is believed to be the exact theory. An interesting attempt in this direction has been made by Mittleman [42, but it is not fully convincing. According to this author, the ground state of the Dirac-Fock model should be obtained by means of a max-min procedure applied to the no-photon QED Hamiltonian $\mathbf{H}_{\mathrm{QED}}$. In this procedure, the reference projector for the normal ordering of $\mathbf{H}_{\mathrm{QED}}$ is not fixed, and the vacuum polarization terms are neglected. Then one has to look for a projector which maximizes, in the Hartree-Fock approximation, the ground-state energy of the normal-ordered Hamiltonian. From a mathematical viewpoint, Mittleman's max-min principle has been investigated in the papers [4, 17, 7, 6]. In the case of zero or one electron [4, 17, it works very well and one shows that the projector $P^{\alpha \varphi}$ of the Furry picture is the optimal reference. But it seems, from the counterexample given in [6], that serious problems occur when there are several electrons.

In their work 9], Chaix and Iracane derive their new mean-field model (which they call Bogoliubov-Dirac-Fock) from the no-photon QED Hamiltonian, normal-ordered with respect to a fixed reference: the free projector $P^{0}$. They keep the vacuum polarization terms, pointing out that they are "necessary for the internal consistency of the relativistic mean-field theory and should therefore be taken into account in proper self-consistent calculations, independently of the magnitude of the physical effects" [9, page 3813]. This allows them to obtain a bounded-below energy: the ground states are simply defined as minimizers and no max-min procedure is needed. A minimizer without charge constraint, if it exists, is a projector satisfying a self-consistent equation: it should be the negative spectral projector of the mean-field Hamiltonian generated by the nuclear charge density, corrected by a vacuum polarization effect. This selfconsistent projector is the stable dressed vacuum. Now, if one restricts the BDF functional to the charge sector $-N$, and if one can find a minimizer, it will be solution of the Dirac-Fock equations for $N$ electrons, corrected by a vacuum polarization term [9, section 4.2]. The Dirac-Fock model is thus interpreted by Chaix-Iracane as a nonvariational approximation of BDF. In other words, the Euler-Lagrange equations only differ by small terms, but the variational structure is completely different since the DF functional is strongly indefinite (i.e., it is not bounded below and all its critical points have an infinite Morse index).

As we have seen, the Chaix-Iracane model has several advantages as compared to the standard Dirac-Fock model: it is more accurate (taking into account vacuum polarization effects), its physical derivation is more convincing, and the ground state solutions have a simple definition as minimizers of the BDF functional. The drawback is that it is not easy to give a meaning to the quantities (energy of the vacuum, charge density of the vacuum) appearing in the BDF model. It is well known that there are divergent quantities in QED even after normal ordering, but Chaix and Iracane do not address this problem in their work. The first rigorous works on the BDF model are due to ChaixIracane-Lions [10] and Bach-Barbaroux-Helffer-Siedentop [4]. In particular, in [4], the authors give a rigorous meaning to the BDF energy in the class of op- 
erators with trace, and show that it is bounded below if one fixes the reference for normal ordering. Then Bach et al. vary the reference for normal ordering and neglect the vacuum polarization terms, which are experimentally small and mathematically divergent. This approximation is exactly the one made by Mittleman [42] in his formal derivation of the Dirac-Fock model. In the present work, our approach is different: we keep $P^{0}$ as reference for normal ordering, we study the full Chaix-Iracane model of the dressed vacuum (without neglecting any divergent term), and we control the divergences thanks to a momentum cut-off. Nevertheless, the paper [4] has been an important source of inspiration in our study: it contains very useful mathematical ideas and results, in particular the lower bound on the energy (see Theorem 1 in the present paper, which is a mere rephrasing, in our framework, of this estimate).

Mathematically speaking, we shall say that a vacuum is an orthogonal projector $P$ with the additional requirement that

$$
Q=P-P^{0} \in \mathfrak{S}_{2}(\mathcal{H})
$$

where $\mathfrak{S}_{2}(\mathcal{H})$ denotes the class of Hilbert-Schmidt operators on $\mathcal{H}$. As explained in the Appendix, this condition guarantees that $P$ is the (unrenormalized) density matrix of a dressed vacuum in the electron-positron Fock space associated with the free projector $P^{0}$. This dressed vacuum may be seen (formally) as an infinite Slater determinant $\Omega=\psi_{1} \wedge \cdots \wedge \psi_{i} \wedge \cdots$ where $\left(\psi_{i}\right)_{i \geq 1}$ is an orthonormal basis of $P \mathcal{H}$. Since the model takes the free vacuum as reference according to Dirac's ideas [13, 14], $Q$ is the true (renormalized) one-body density matrix of $\Omega$. Following [9] (with notations from [4]), the BDF energy of the dressed vacuum can be written (formally) as follows, as a function of its renormalized density matrix :

$\mathcal{E}(Q)=\operatorname{tr}\left(D^{0} Q\right)-\alpha \int \rho_{Q} \varphi+\frac{\alpha}{2} \iint \frac{\rho_{Q}(x) \rho_{Q}(y)}{|x-y|} d x d y-\frac{\alpha}{2} \iint \frac{|Q(x, y)|^{2}}{|x-y|} d x d y$

with $\rho_{Q}(x)=\operatorname{tr}_{\mathbb{C}^{4}} Q(x, x)$. By formal computations, Chaix and Iracane 9, Section 4.2] show that the Euler-Lagrange equation of this functional is

$$
\left[P, D_{Q}\right]=0
$$

where

$$
D_{Q}:=D^{\alpha \varphi}+\alpha \rho_{Q} * \frac{1}{|\cdot|}-\alpha \frac{Q(x, y)}{|x-y|},
$$

$Q=P-P^{0}$. For a minimizer, the second order condition implies a more precise relation between $P$ and $D_{Q}$, which takes the form of a fixed-point equation:

$$
P=\chi_{(-\infty ; 0)}\left(D_{Q}\right)=\chi_{(-\infty ; 0)}\left(D^{\alpha \varphi}+\alpha \rho_{Q} * \frac{1}{|\cdot|}-\alpha \frac{Q(x, y)}{|x-y|}\right) .
$$

Remark that if $\varphi=0$ (no external potential), then $P^{0}$ is already a solution of this equation since $Q=0$ in this particular case. 
The present paper contains the first mathematical study of a fixed-point algorithm for finding a solution of (6). Notice that the use of a fixed-point method to solve a self-consistent equation is very common in quantum chemistry and physics and that most of the numerical algorithms used in practice are based on this idea. For a mathematical existence result using the Schauder fixedpoint theorem, see the resolution of the Hartree equations in [57]. See also [8], where rigorous results are given on the convergence of standard Hartree-Fock iteration schemes. For the determination of a projector in no-photon QED, the fixed-point method has been used for the first time by Lieb and Siedentop 39. Their goal was to replace $P^{0}$ by a new (self-consistent) projector commuting with translations, as reference for normal ordering in the absence of external field. We use the Banach fixed-point theorem as in [39], but our physical model is very different, and the necessary estimates are much more delicate in our case.

Of course, we have to make an assumption on the external potential: it should have a certain regularity, and should not be too strong, otherwise we are not able to prove that the iteration method converges. If one is only interested in the existence of a minimizer, it is possible to remove the smallness assumption on the potential, but for this purpose the constructive fixed-point approach must be replaced by a direct - and non-constructive - minimization argument 28 . The regularity assumption cannot be dropped: this is a well known phenomenon in QED when $P^{0}$ is chosen as reference for normal ordering (see e.g., 35]). But this regularity is not really a restriction from the point of view of physics: point-like nuclei do not exist in nature.

In [4, the operator $D^{0} Q$ is assumed to be trace class, so that the expressions (31) and (15) are well defined. Unfortunately, it turns out that, when $\varphi$ is nonzero, $Q=P-P^{0}$ is never trace class if $P$ is a solution of ([6). Therefore no minimizer can exist in the trace class $\mathfrak{S}_{1}(\mathcal{H})$ in the presence of an external field. So we must try to define the BDF energy and the self-consistent equation for operators which are not trace class, and this leads to several difficulties.

A first problem occurs with the definition of $\operatorname{tr}\left(D^{0} Q\right)$ in (3). To solve it, we will have to extend the trace functional to a bigger class of compact operators, namely the operators with " $P^{0}$-trace" (see Section 2.1 below).

A second problem occurs with the definition of the density $\rho_{Q}$. For this reason, we introduce a momentum cut-off $\Lambda$, which means we replace the ambient space $\mathcal{H}$ by

$$
\mathcal{H}_{\Lambda}:=\{f \in \mathcal{H}, \operatorname{supp}(\widehat{f}) \subset B(0, \Lambda)\} .
$$

Since $D^{0}$ is a multiplication operator in Fourier space, $\mathcal{H}_{\Lambda}$ is invariant under $P^{0}$ and we keep the notation $P^{0}$ for the restricted operator. With the cut-off, the integral kernel $Q(x, y)$ of $P-P^{0} \in \mathfrak{S}_{2}\left(\mathcal{H}_{\Lambda}\right)$ becomes smooth for any dressed vacuum $P$, and one can easily define $\rho_{Q}(x)=\operatorname{tr}_{\mathbb{C}^{4}} Q(x, x)$. Notice that, even with our ultraviolet cut-off, $Q=P-P^{0}$ is never trace class if $P$ is a solution of (6) and if an external potential is present. As we shall see it later on, our results will be valid under a technical condition of the form $\alpha \sqrt{\ln \Lambda} \leq C$ for some constant $C$. For a small $\alpha$, this leads to an extremely large $\Lambda$, which corresponds 
to scales that are far beyond the reach of experimental and theoretical physics at the present time. But our conditions do not allow to pass to the limit of an infinite cut-off.

Note that if one expands the right-hand side of equation (6) in powers of the small parameter $\alpha$, the first order term contains an expression which diverges logarithmically as $\Lambda$ goes to infinity. When the exchange term $Q(x, y) /|x-y|$ is neglected, a simple algebraic manipulation allows to rewrite a posteriori our cut-off version of equation (6) in a renormalized form, with the divergent term removed, and the "bare" constant $\alpha$ in front of the charge densities replaced by a smaller, "dressed", coupling constant

$$
\alpha_{\mathrm{dr}} \simeq \frac{\alpha}{1+\frac{2 \alpha}{3 \pi} \log \Lambda}
$$

(details will be given in a forthcoming paper [28]). The dressed constant is the observable one. Its experimental value is $\alpha_{\mathrm{dr}} \simeq 1 / 137$. This kind of "charge renormalization" associated with a momentum cut-off is standard in the physics literature (see, e.g., 33, Equation (7.18)]). With this interpretation, the limit case of an infinite cut-off appears as unphysical (it would correspond to $\alpha_{\mathrm{dr}}=0$, which means no more electrostatic interaction).

Remark 1. In the Furry picture, that is to say when $P=P^{\alpha \varphi}$, it is known since the very beginning of QED [14, 31, 23, 55, 51] that the density $\rho^{\alpha \varphi}$ associated with $Q^{\alpha \varphi}=P^{\alpha \varphi}-P^{0}$ is never well-defined if no ultraviolet cut-off is imposed. One possible regularization procedure [19, 37, 29] is to remove the divergent part of $\rho^{\alpha \varphi}$, which is (formally) proportional to the nuclear charge density $n$. This gives a renormalized density $\rho_{\mathrm{ren}}^{\alpha \varphi}$ which can be defined without the help of a high momentum cut-off. This procedure has recently been clarified by Hainzl and Siedentop in 29]. Some interesting features of $\rho_{\mathrm{ren}}^{\alpha \varphi}$, in the case of strong external fields, were obtained by Hainzl in [27. We do not want to give a precise definition of $\rho_{\mathrm{ren}}^{\alpha \varphi}$ here and we refer the reader to [29, 27].

It would be tempting, instead of using a cut-off, to renormalize $\rho_{Q}$ a priori in equation ([6), as in [29]. But we do not know how to solve the resulting renormalized equation (6) if no cut-off is made. Moreover, even if we could find a solution without momentum cut-off, its interpretation as a minimizer of the BDF energy would be unclear.

The paper is organized as follows. In the next section, we define the Bogoliubov-Dirac-Fock model and state our main results. For the sake of clarity, we have brought all the proofs together in Sections 3 and 4 . In the Appendix, we explain in our language, for the reader's convenience, how the BDF energy is deduced from no-photon QED by Chaix-Iracane in [9].

Acknowledgment: C.H. wishes to thank Heinz Siedentop for suggesting him the possibility of studying a self-consistent model of the polarized vacuum, during his time as post-doc at the LMU (Munich). The authors acknowledge support from the European Union's IHP network Analysis and Quantum HPRNCT-2002-00277. E.S. acknowledges support from the Institut Universitaire de France. 


\section{Model and main results}

In this section, we study the Bogoliubov-Dirac-Fock model introduced in [9, 11]. Our system of notation is similar to [4, with the difference that we keep all the terms describing the vacuum polarization. This forces us to deal with operators which are not trace class, unlike [4].

\subsection{An extension of the trace functional}

In order to give a meaning to the expression " $\operatorname{tr}\left(D^{0} Q\right)$ " even when $Q$ is not trace-class, we need the notion of " $P^{0}$-trace". In this section only, we work in an abstract Hilbert space $\mathfrak{h}$.

Definition 1. Let $P$ be a projector such that $P$ and $1-P$ have infinite rank, and $A \in \mathfrak{S}_{2}(\mathfrak{h})$. We shall say that $A$ is $P$-trace class if and only if $A_{++}:=$ $(1-P) A(1-P)$ and $A_{--}:=P A P$ are trace class. Then we define the $P$-trace of $A$ by

$$
\operatorname{tr}_{P}(A):=\operatorname{tr}\left(A_{++}\right)+\operatorname{tr}\left(A_{--}\right) .
$$

We denote by $\mathfrak{S}_{1}^{P}(\mathfrak{h})$ the space of all Hilbert-Schmidt operators which are $P$ trace class.

Notice that if $A$ is a trace class operator, then $A \in \mathfrak{S}_{1}^{P}(\mathfrak{h})$ and $\operatorname{tr}(A)=$ $\operatorname{tr}_{P}(A)$ for any projector $P$.

Remark 2. In [54, Section 5.7.2], a similar definition in connection with supersymmetry is made and the name "supertrace" is used.

The following result, whose proof is given in Section 3 will be used repeatedly in the sequel.

Lemma 1. Let $P$ and $P^{\prime}$ be two projectors such that $P-P^{\prime} \in \mathfrak{S}_{2}(\mathfrak{h})$. Then $A$ is $P$-trace class if and only if it is $P^{\prime}$-trace class, and in this case $\operatorname{tr}_{P}(A)=$ $\operatorname{tr}_{P^{\prime}}(A)$.

Another useful fact is that when $A$ is Hilbert-Schmidt and $A+P$ is a projector, then $A$ has a $P$-trace, as explained below:

Lemma 2. Let $P$ and $P^{\prime}$ be two projectors on a Hilbert space, such that $P^{\prime}-P$ is a Hilbert-Schmidt operator. Then $P^{\prime}-P$ is $P$-trace class. Moreover, $\operatorname{tr}_{P}\left(P^{\prime}-P\right)$ is an integer which satisfies

$$
\operatorname{tr}_{P}\left(P^{\prime}-P\right)=\operatorname{tr}\left(\left(P^{\prime}-P\right)^{2 n+1}\right)
$$

for all $n \geq 1$, and $\operatorname{tr}_{P}\left(P^{\prime}-P\right)=0$ when $\left\|P^{\prime}-P\right\|_{\mathfrak{S}_{\infty}}<1$.

In our framework, a consequence is that, for any vacuum $P$ such that $Q=$ $P-P^{0} \in \mathfrak{S}_{2}\left(\mathcal{H}_{\Lambda}\right), \operatorname{tr}_{P^{0}}(Q)$ is an integer which can be interpreted as the charge of the dressed vacuum $P$ (see the Appendix for comments in this direction). When $P$ solves the self-consistent equation (6) and $\varphi$ is not too strong, we will see that $P$ is close to $P^{0}$, so that its charge will be zero, according to the lemma. 


\subsection{The Bogoliubov-Dirac-Fock model}

As in 4], we are going to extend the BDF energy to a convex set of compact operators, which can be interpreted as one-particle density matrices of quasifree states. This kind of extension is standard for mean-field models depending only on the one-body density matrix (see [38, 3, 5]).

In the whole paper, we assume that the nuclear charge density $n=-\Delta \varphi / 4 \pi$ belongs to the Hilbert space

$$
\mathfrak{C}=\left\{f \in L^{2}\left(\mathbb{R}^{3}, \mathbb{R}\right), D(f, f)<\infty\right\},
$$

where

$$
D(f, g)=4 \pi \int \frac{\overline{\widehat{f}(k)} \widehat{g}(k)}{|k|^{2}} d k .
$$

We will choose the following Hilbert norm on $\mathfrak{C}$ :

$$
\|f\|_{\mathfrak{C}}:=\left(\int \frac{1+|k|^{2}}{|k|^{2}}|\widehat{f}(k)|^{2} d k\right)^{1 / 2} .
$$

The Bogoliubov-Dirac-Fock energy is defined by

$$
\mathcal{E}(\Gamma)=\operatorname{tr}_{P^{0}}\left(D^{0} \Gamma\right)-\alpha D\left(\rho_{\Gamma}, n\right)+\frac{\alpha}{2} D\left(\rho_{\Gamma}, \rho_{\Gamma}\right)-\frac{\alpha}{2} \iint \frac{|\Gamma(x, y)|^{2}}{|x-y|} d x d y
$$

on the set

$$
\mathcal{G}_{\Lambda}:=\left\{\Gamma \in \mathfrak{S}_{1}^{P^{0}}\left(\mathcal{H}_{\Lambda}\right) \mid-P^{0} \leq \Gamma \leq 1-P^{0}, \rho_{\Gamma} \in \mathfrak{C}\right\}
$$

In (7),

$$
\widehat{\rho}_{\Gamma}(k)=\frac{1}{(2 \pi)^{3 / 2}} \int_{|p| \leq \Lambda} \operatorname{Tr}_{\mathbb{C}^{4}}(\widehat{\Gamma}(p+k / 2, p-k / 2)) d p
$$

is the Fourier transform of the charge density $\rho_{\Gamma}$, which, formally, is the diagonal of $\Gamma \in \mathfrak{S}_{2}\left(\mathcal{H}_{\Lambda}\right)$, as explained in the Introduction. Thanks to the momentum cut-off, $\widehat{\rho_{\Gamma}}$ is compactly supported, so that $\widehat{\rho}_{\Gamma} \in L^{1}$, hence

$$
\rho_{\Gamma}(x)=\operatorname{Tr}_{\mathbb{C}^{4}}(\Gamma(x, x))=\frac{1}{(2 \pi)^{3}} \iint_{|p|,|q| \leq \Lambda} \operatorname{Tr}_{\mathbb{C}^{4}}(\widehat{\Gamma}(p, q)) e^{i x(p-q)} d p d q .
$$

Clearly, the function $\Gamma \in \mathfrak{S}_{2}\left(\mathcal{H}_{\Lambda}\right) \mapsto \rho_{\Gamma} \in \mathcal{C}_{0}^{0} \cap L^{2}\left(\mathbb{R}^{3}\right)$ is continuous. Notice that if for instance $f, g \in H^{1}\left(\mathbb{R}^{3}, \mathbb{R}\right)$, then the electrostatic energy is simply

$$
D(f, g)=\iint_{\mathbb{R}^{3} \times \mathbb{R}^{3}} \frac{f(x) g(y)}{|x-y|} d x d y
$$

but for functions in $\mathfrak{C}$, it does not necessarily have a meaning as a Lebesgue double integral in direct space.

Note that the set $\mathcal{G}_{\Lambda}$ is convex, and that the elements of $P^{0}+\mathcal{G}_{\Lambda}$ are not necessarily projectors. In fact, it is an easy exercise to show that an element of $\mathcal{G}_{\Lambda}$ is extremal if and only if it is of the form $P-P^{0}$, with $P$ a projector. It 
then follows from Lemma 2 that the set of all extremal points of $\mathcal{G}_{\Lambda}$ coincides with $\mathcal{Q}_{\Lambda}=\mathcal{P}_{\Lambda}-P^{0}$, where

$$
\mathcal{P}_{\Lambda}=\left\{P \text { orth. projector } \mid Q=P-P^{0} \in \mathfrak{S}_{2}\left(\mathcal{H}_{\Lambda}\right), \rho_{Q} \in \mathfrak{C}\right\} .
$$

It will turn out that, under some assumptions on $\alpha, \Lambda$ and $n$, the BDF functional has a unique minimizer on $\mathcal{G}_{\Lambda}$ which is extremal. As a consequence,

$$
\inf \left\{\mathcal{E}\left(P-P^{0}\right), P \in \mathcal{P}_{\Lambda}\right\}=\inf \left\{\mathcal{E}(\Gamma), \Gamma \in \mathcal{G}_{\Lambda}\right\}
$$

In the next subsection, we give necessary and sufficient conditions satisfied by a minimizer of $\mathcal{E}$.

\subsection{Study of the BDF energy}

We first state the following result, which is an easy translation, in our framework, of the stability estimate proved by Bach et al [4] (see also [10]):

Theorem 1. Let be $n \in \mathfrak{C}$. Then

1. $\mathcal{E}$ is well-defined on $\mathcal{G}_{\Lambda}$;

2. if $0 \leq \alpha \leq \frac{4}{\pi}$, then

$$
\forall \Gamma \in \mathcal{G}_{\Lambda}, \quad \mathcal{E}(\Gamma)+\frac{\alpha}{2} D(n, n) \geq 0
$$

and therefore $\mathcal{E}$ is bounded from below on $\mathcal{G}_{\Lambda}$, independently of $\Lambda$;

3. if $0 \leq \alpha \leq \frac{4}{\pi}$ and $n=0$, then $\mathcal{E}$ is non-negative on $\mathcal{G}_{\Lambda}$ [4, 10], 0 being the unique minimizer.

Remark 3. Note that the result is optimal in the sense that the functional becomes unbounded from below when $n=0$ if $\alpha>4 / \pi$, as shown in [10] and 32 .

Remark 4. Since $\alpha D(n, n) / 2$ in (10) is the electrostatic energy of the field created by $n$, (10) means that the total energy of the system is nonnegative.

Proof of Theorem [1. We only explain here why $\mathcal{E}$ is well defined on $\mathcal{G}_{\Lambda}$, the rest being identical to the proof of Theorem 1 in [4] (see [4, Eq. (18)-(19)]).

If $\Gamma \in \mathfrak{S}_{1}^{P^{0}}\left(\mathcal{H}_{\Lambda}\right)$, then we have

$$
P^{0} D^{0} \Gamma P^{0}=D^{0} P^{0} \Gamma P^{0}=D^{0} \Gamma_{++} \in \mathfrak{S}_{1}\left(\mathcal{H}_{\Lambda}\right)
$$

since $P^{0}$ commutes with $D^{0}$ and $\left|D^{0}\right| \leq \sqrt{1+\Lambda^{2}}$. With a similar argument for $1-P^{0}$, we obtain that $D^{0} \Gamma \in \mathfrak{S}_{1}^{P^{0}}\left(\mathcal{H}_{\Lambda}\right)$. Therefore, $\operatorname{tr}_{P^{0}}\left(D^{0} \Gamma\right)$ is well-defined and

$$
\operatorname{tr}_{P^{0}}\left(D^{0} \Gamma\right)=\operatorname{tr}\left(D^{0} \Gamma_{++}\right)+\operatorname{tr}\left(D^{0} \Gamma_{--}\right)=\operatorname{tr}\left(\left|D^{0}\right| \Gamma_{++}\right)-\operatorname{tr}\left(\left|D^{0}\right| \Gamma_{--}\right)
$$


(notice that, due to the constraint $-P^{0} \leq \Gamma \leq 1-P^{0}$, one has $\Gamma_{++} \geq 0$ and $\left.\Gamma_{--} \leq 0\right)$. On the other hand we have by Kato's inequality

$$
\iint \frac{|\Gamma(x, y)|^{2}}{|x-y|} d x d y \leq \frac{\pi}{2} \operatorname{tr}\left(\left|D^{0}\right| \Gamma^{2}\right)
$$

showing that this last term is well-defined since $\left|D^{0}\right|$ is bounded on $\mathcal{H}_{\Lambda}$ and $\Gamma \in \mathfrak{S}_{2}\left(\mathcal{H}_{\Lambda}\right)$.

We are interested in minimizers of the BDF functional, and we expect them to be in the class $\mathcal{Q}_{\Lambda}=\mathcal{P}_{\Lambda}-P^{0}$. This leads to the following definition

Definition 2. We say that a projector $P$ is a BDF-stable vacuum if and only if $P-P^{0}$ is a minimizer of $\mathcal{E}$ on $\mathcal{G}_{\Lambda}$.

When there is no external potential, $P^{0}$ is the unique BDF-stable vacuum [10, 4], which corresponds to Dirac's ideas. But if we consider a non-vanishing external potential $\varphi=n * \frac{1}{\mid \cdot}$, then $P^{0}$ obviously cannot be BDF-stable, since it is easy to create a state $-P^{0} \leq \gamma \leq 1-P^{0}$ such that $\mathcal{E}(\gamma)<0=\mathcal{E}(0)$. This means that the vacuum is necessarily polarized.

More precisely, one can easily derive necessary conditions satisfied by a BDF-stable vacuum $P$. To this end, a perturbation of the form $Q+\gamma=$ $P-P^{0}+\gamma$, with $\gamma \in \mathfrak{S}_{1}\left(\mathcal{H}_{\Lambda}\right)$ such that $-P \leq \gamma \leq 1-P$ is considered in Chaix-Iracane [9, formula (4.8)], and the energy $\mathcal{E}(Q+\gamma)$ is expanded to get

$$
\mathcal{E}(Q+\gamma)=\operatorname{tr}\left(D_{Q} \gamma\right)+\frac{\alpha}{2} D\left(\rho_{\gamma}, \rho_{\gamma}\right)-\frac{\alpha}{2} \iint \frac{|\gamma(x, y)|^{2}}{|x-y|} d x d y+\mathcal{E}(Q),
$$

a formula which is valid when $\gamma \in \mathfrak{S}_{1}\left(\mathcal{H}_{\Lambda}\right)$, the operator $D_{Q}$ being defined in (51).

Remark 5. In [4, Formula (21)] and [7, the polarization potentials appearing in $D_{Q}$ and the energy of the vacuum $\mathcal{E}(Q)$ were neglected by the authors who used the following functional

$$
\tilde{\mathcal{E}}_{P}(\gamma)=\operatorname{tr}\left(D^{\alpha \varphi} \gamma\right)+\frac{\alpha}{2} D\left(\rho_{\gamma}, \rho_{\gamma}\right)-\frac{\alpha}{2} \iint \frac{|\gamma(x, y)|^{2}}{|x-y|} d x d y
$$

with the constraints $\gamma \in \mathfrak{S}_{1}$ and $-P \leq \gamma \leq 1-P$ (and even $P \gamma(1-P)=0$ in [7]). Then a procedure taking the form $\sup _{P} \inf _{-P \leq \gamma \leq 1-P} \tilde{\mathcal{E}}_{P}(\gamma)$, related to Mittleman's work [42], was considered in [4]. For the case of the vacuum (no constraint on the trace of $\gamma$ ), the solution is the Furry picture $P=P^{\alpha \varphi}$ with $\gamma=0$, as shown in [4. We refer the reader to [9, page 3809] and [17, 6, 7] for comments and results concerning Mittleman's max-min in the case of $N$ electrons (which corresponds to the additional constraint $\operatorname{tr}(\gamma)=N$ ).

¿From formula (12), it can be seen that a BDF-stable vacuum must satisfy the fixed-point equation (6). The converse is also true under some assumptions: 
Theorem 2 (BDF-Stability). Let be $P \in \mathcal{P}_{\Lambda}$ and $n \in \mathfrak{C}$. We assume that there exists a positive constant $d$ such that

$$
d\left|D_{Q}\right| \geq\left|D^{0}\right| \text { with } \alpha d \frac{\pi}{4} \leq 1
$$

where $D_{Q}$ is defined in (5). Then, the following assertions are equivalent

1. $P$ fulfills the equation

$$
P=\chi_{(-\infty ; 0)}\left(D_{Q}\right)=\chi_{(-\infty ; 0)}\left(D^{\alpha \varphi}+\alpha \rho_{Q} * \frac{1}{|\cdot|}-\alpha \frac{Q(x, y)}{|x-y|}\right) .
$$

2. $P$ is the unique BDF-stable vacuum, i.e. $P-P^{0}$ is the unique global minimizer of $\mathcal{E}$ on $\mathcal{G}_{\Lambda}$.

The proof of this theorem will be given in Section 3. Some arguments are directly inspired of 4 .

Remark 6. One can try to go further: if we translate the ideas of Chaix and Iracane in our language, the ground state of a molecule consisting of nuclei with total charge density $n$, surrounded by a cloud of $N$ electrons, should solve the following constrained minimization problem

$$
\min \left\{\mathcal{E}(\Gamma), \Gamma \in \mathcal{G}_{\Lambda}, \operatorname{tr}_{P^{0}}(\Gamma)=N\right\}
$$

with $N \in \mathbb{N} \backslash\{0\}$. If this minimization problem has a solution $Q$, it will solve a self-consistent equation of the form

$$
Q=\chi_{(-\infty ; \mu)}\left(D_{Q}\right)-P^{0}
$$

where $\mu \in(-1 ; 1)$ is a Lagrange multiplier associated with the charge constraint, and interpreted as a chemical potential. For a not too strong external field $\varphi$, it should be possible to prove that $\mu \in(0 ; 1)$ and that the vacuum $\Pi=$ $\chi_{(-\infty ; 0)}\left(D_{Q}\right)$ stays neutral, which means $\operatorname{tr}_{P^{0}}\left(\Pi-P^{0}\right)=0$. Therefore, we could split $P=Q+P^{0}=\chi_{(-\infty ; \mu)}\left(D_{Q}\right)$ in the form

$$
P=\Pi+\sum_{k=1}^{N}\left|\psi_{k}\right\rangle\left\langle\psi_{k}\right| .
$$

The mono-electronic wave functions $\psi_{k}$ would be solutions of the Dirac-Fock equations (with high momentum cut-off), perturbed by vacuum polarization terms:

$$
D_{Q} \psi_{k}=\varepsilon_{k} \psi_{k}, \quad 0<\varepsilon_{k}<1, \quad 1 \leq k \leq N .
$$

It is our goal to study this constrained variational problem in the near future. The present work, which deals with the unconstrained case, is a first step in this direction. 


\subsection{Existence of a BDF-stable vacuum}

We may now state our main Theorem. We recall that the norm on $\mathfrak{C}$ is

$$
\|f\|_{\mathfrak{C}}:=\left(\int \frac{1+|k|^{2}}{|k|^{2}}|\widehat{f}(k)|^{2} d k\right)^{1 / 2}
$$

Theorem 3 (Existence of a BDF-stable vacuum). Let be $n \in \mathfrak{C}$ and $b \in(0 ; 1)$. Then for all $\Lambda$ and $\alpha$ such that

$$
2 \sqrt{\pi} \alpha\|n\|_{\mathfrak{C}} \leq b \quad \text { and } \quad \alpha \leq \alpha_{b}(\Lambda)
$$

where

$$
\alpha_{b}(\Lambda) \sim_{\Lambda \rightarrow \infty} \frac{C(1-b)}{\sqrt{\log \Lambda}},
$$

there exists a unique BDF-stable vacuum $P$, which is a solution of

$$
P=\chi_{(-\infty ; 0)}\left(D_{Q}\right)=\chi_{(-\infty ; 0)}\left(D^{0}+\alpha\left(\rho_{Q}-n\right) * \frac{1}{|\cdot|}-\alpha \frac{Q(x, y)}{|x-y|}\right)
$$

with $Q=P-P^{0}$. Moreover, we have $\operatorname{tr}_{P^{0}}(Q)=0$.

Remark 7. The first constraint $2 \sqrt{\pi} \alpha\|n\|_{\mathfrak{C}} \leq b$ means that the external field is not too strong. It explains why a neutral polarized vacuum is obtained (since $\left.\operatorname{tr}_{P^{0}}(Q)=0\right)$. In our proof, this constraint on the external field is necessary for the fixed point algorithm to converge. The second constraint $\alpha \leq \alpha_{b}(\Lambda)$, which essentially reduces to $\alpha \sqrt{\log \Lambda} \lesssim C(1-b)$, is a technical condition due to our choice of norms, but we were unable to drop it. It disappears if the exchange term $\frac{\alpha}{2} \iint \frac{|Q(x, y)|^{2}}{|x-y|} d x d y$ is neglected in the energy, as can be seen from the proof. A precise definition of the constant $C$ appearing in this result is given in the proof.

Remark 8. There is an interesting symmetry property of the solutions of (17) when $n$ is replaced by $-n$. Namely, if $P$ is a solution of (17) with external density $n$, then $P^{\prime}=Q^{\prime}+P^{0}$ is a solution of (17) with external density $-n$, where $Q^{\prime}=-C Q C^{-1}, C$ being the charge conjugation operator [54, page 14]. The two dressed vacua $P$ and $P^{\prime}$ have the same BDF energies and satisfy $\rho_{Q^{\prime}}=-\rho_{Q}$, as suggested by the intuition. For this symmetry between matter and antimatter to be true, it is essential to have the Fermi level at 0 and not at -1 (see, e.g., the comments of [4, page 197] about this fact).

\subsection{Idea of the proof of Theorem 3; the fixed-point algorithm}

We end this section with a brief description of our fixed-point algorithm, used in the proof of Theorem 3, and which could be useful for practical computations.

A natural scheme for solving (17) would be to construct a sequence $\left(Q_{j}\right)_{j \geq 0} \subset$ $\mathcal{G}_{\Lambda}$ by taking $Q_{0}=0$ and

$$
Q_{j+1}=\chi_{(-\infty ; 0)}\left(D^{0}+\alpha\left(\rho_{Q_{j}}-n\right) * \frac{1}{|\cdot|}-\alpha \frac{Q_{j}(x, y)}{|x-y|}\right)-P^{0} .
$$


Expanding this expression in powers of $\alpha$, and considering the total density

$$
\rho_{Q_{j}}^{\prime}:=\rho_{Q_{j}}-n
$$

one can write the following recursion formula in Fourier space:

$$
\widehat{\rho_{Q_{j+1}}^{\prime}}(k)=-\widehat{n}(k)-\alpha B_{\Lambda}(k) \widehat{\rho_{Q_{j}}^{\prime}}(k)+\alpha \widehat{\rho_{1,0}\left(Q_{j}\right)}(k)+\sum_{n=2}^{\infty} \alpha^{n} \widehat{\left.\rho_{n} \widehat{\left(Q_{j}, \rho_{Q_{j}}^{\prime}\right.}\right)}(k) .
$$

The notations $B_{\Lambda}, \rho_{1,0}$ and $\rho_{n}$ are defined precisely in Section 4.1 (see the subsections 4.1.3 and 4.1.4). The important point is that $B_{\Lambda}(k)$ is a positive function which diverges logarithmically as $\Lambda \rightarrow \infty$ for any fixed $k$, whereas the other terms stay bounded. From (19) we thus see that the scheme (18) would converge under a condition of the form $\alpha \log \Lambda \leq C$.

To improve this condition, we use a better algorithm in our proof. Our modified scheme consists in defining a sequence of pairs $\left(Q_{j}, \rho_{j}^{\prime}\right)_{j \geq 0} \subset \mathcal{G}_{\Lambda} \times \mathfrak{C}$ such that $\left(Q_{0}, \rho_{0}^{\prime}\right)=(0,-n)$ and

$$
\left\{\begin{array}{l}
Q_{j+1}=\chi_{(-\infty ; 0)}\left(D^{0}+\alpha \rho_{j}^{\prime} * \frac{1}{|\cdot|}-\alpha \frac{Q_{j}(x, y)}{|x-y|}\right)-P^{0} \\
\rho_{j+1}^{\prime}=L(\alpha, \Lambda) \rho_{Q_{j+1}}^{\prime}+(1-L(\alpha, \Lambda)) \rho_{j}^{\prime}
\end{array}\right.
$$

where $\rho_{Q_{j}}^{\prime}(x):=\rho_{Q_{j}}(x)-n(x)=\operatorname{tr}_{\mathbb{C}^{4}} Q_{j}(x, x)-n(x)$, and $L(\alpha, \Lambda)$ is the linear operator which, in the Fourier domain, is just the multiplication by the function $\left(1+\alpha B_{\Lambda}(k)\right)^{-1}$. The second equation in the iteration scheme (20) can be written in the form

$$
\widehat{\rho_{j+1}^{\prime}}(k)=\left(1+\alpha B_{\Lambda}(k)\right)^{-1}\left[-\widehat{n}(k)+\alpha \widehat{\rho_{1,0}\left(Q_{j}\right)}(k)+\sum_{n=2}^{\infty} \alpha^{n} \widehat{\rho_{n\left(Q_{j}, \rho_{j}^{\prime}\right)}}(k)\right]
$$

The divergent term now only appears in the denominator. So one expects a much better convergence. In the proof of Theorem 3, we show the convergence of the algorithm (20) under the conditions (16) but we believe that it converges independently of the cut-off $\Lambda$.

It can be seen from our proof that this holds when the exchange term $\alpha \frac{Q_{j}(x, y)}{|x-y|}$ is neglected in (20). In this case, the algorithm converges independently of $\Lambda$ to the solution of a reduced fixed-point problem (without exchange term), which is the unique minimizer on $\mathcal{G}_{\Lambda}$ of the convex functional

$$
\mathcal{E}_{\text {red }}(\Gamma)=\operatorname{tr}_{P^{0}}\left(D^{0} \Gamma\right)-\alpha D\left(\rho_{\Gamma}, n\right)+\frac{\alpha}{2} D\left(\rho_{\Gamma}, \rho_{\Gamma}\right)
$$

\section{Proof of Theorem 2}

In this section, we prove Theorem 2. To this end, we first need to prove Lemmas [1] and 2 


\subsection{Proof of Lemmas [1] and 2}

Proof of Lemma 1. Let be $P$ and $P^{\prime}$ two projectors such that $P^{\prime}-P \in \mathfrak{S}_{2}(\mathfrak{h})$, and a Hilbert-Schmidt operator $A$ which is $P$-trace class. This means that $P A P$ and $(1-P) A(1-P)$ are trace class.

Let us first show that $P^{\prime} A P^{\prime}$ is trace class. To this end, we write

$$
\begin{aligned}
P^{\prime} A P^{\prime} & =\left(P^{\prime}-P+P\right) A\left(P^{\prime}-P+P\right) \\
& =\left(P^{\prime}-P\right) A\left(P^{\prime}-P\right)+\left(P^{\prime}-P\right) A P+P A\left(P^{\prime}-P\right)+P A P .
\end{aligned}
$$

This shows that $P^{\prime} A P^{\prime}$ is trace class since the last term is in $\mathfrak{S}_{1}$ by assumption, $P^{\prime}-P$ and $A$ are in $\mathfrak{S}_{2}$, and $P$ is bounded. The same computation shows that $\left(1-P^{\prime}\right) A\left(1-P^{\prime}\right)$ is trace class.

We now compute

$$
\begin{aligned}
\operatorname{tr}\left[P^{\prime} A P^{\prime}\right] & =\operatorname{tr}\left[\left(P^{\prime}-P\right) A\left(P^{\prime}-P\right)\right]+\operatorname{tr}\left[\left(P^{\prime}-P\right) A P\right]+\operatorname{tr}\left[P A\left(P^{\prime}-P\right)\right]+\operatorname{tr}[P A P] \\
& =\operatorname{tr}\left[A\left(\left(P^{\prime}-P\right)\left(P^{\prime}-P\right)+P\left(P^{\prime}-P\right)+\left(P^{\prime}-P\right) P\right)\right]+\operatorname{tr}[P A P] \\
& =\operatorname{tr}\left[A\left(P^{\prime}-P\right)\right]+\operatorname{tr}[P A P]
\end{aligned}
$$

where we have used the formula $\operatorname{tr}(A B)=\operatorname{tr}(B A)$, valid for $A, B \in \mathfrak{S}_{2}$. The same computation gives

$$
\operatorname{tr}\left[P_{+}^{\prime} A P_{+}^{\prime}\right]=\operatorname{tr}\left[A\left(P_{+}^{\prime}-P_{+}\right)\right]+\operatorname{tr}\left[P_{+} A P_{+}\right]=-\operatorname{tr}\left[A\left(P^{\prime}-P\right)\right]+\operatorname{tr}\left[P_{+} A P_{+}\right]
$$

where we have used the notation $P_{+}=1-P$ and $P_{+}^{\prime}=1-P^{\prime}$. Summing this two results, we obtain the formula $\operatorname{tr}_{P}[A]=\operatorname{tr}_{P^{\prime}}[A]$.

Proof of Lemma 2. We introduce $B=P^{\prime}-P$. We have $B^{2}=P^{\prime}-P^{\prime} P-P P^{\prime}+$ $P=(1-P) B(1-P)-P B P$. This implies that $(1-P) B(1-P)$ and $-P B P$ are non-negative trace class operators. We now use the proof of [2, Theorem 4.1]. Since $B \in \mathfrak{S}_{2}$, we infer $B^{3} \in \mathfrak{S}_{1}$ and so $\left(P^{\prime}, P\right)$ is a Fredholm pair, in the language of [2]. Therefore, $\operatorname{tr}\left(B^{3}\right)$ is an integer and satisfies $\operatorname{tr}\left(B^{3}\right)=\operatorname{tr}\left(B^{2 n+1}\right)$ for all $n \geq 1$. Now we have

$$
B^{3}=B^{2} P^{\prime}-B^{2} P=P^{\prime} B P^{\prime}+P B P .
$$

Applying this result to $1-P^{\prime}$ and $1-P$, we find

$$
B^{3}=\left(1-P^{\prime}\right) B\left(1-P^{\prime}\right)+(1-P) B(1-P) .
$$

Summing this two identities, we obtain by Lemma 1

$$
2 \operatorname{tr}\left(B^{3}\right)=\operatorname{tr}_{P^{\prime}}(B)+\operatorname{tr}_{P}(B)=2 \operatorname{tr}_{P}(B) .
$$

This shows that $\operatorname{tr}_{P}(B)$ indeed equals the index of the pair of projectors $\left(P^{\prime}, P\right)$ defined in 2], an integer which vanishes when $\left\|P^{\prime}-P\right\|_{\mathfrak{S}_{\infty}}<1$ by the results of [2]. 


\subsection{Preliminaries}

To prove Theorem 2 we also need the following

Lemma 3. Assume that $\varphi=\rho * \frac{1}{|\cdot|}$ for some $\rho \in \mathfrak{C}$. Then

$$
\|\nabla \varphi\|_{H^{1}}=4 \pi\|\rho\|_{\mathfrak{C}}, \quad\|\varphi\|_{L^{\infty}} \leq C_{\infty} 4 \pi\|\rho\|_{\mathfrak{C}}, \quad\|\varphi\|_{L^{6}} \leq C_{6} 4 \pi\|\rho\|_{\mathfrak{C}}
$$

where $C_{\infty}:=\frac{1}{2 \pi^{1 / 2}}$ and $C_{6}$ is the Sobolev constant for the inequality $\|u\|_{L^{6}\left(\mathbb{R}^{3}\right)} \leq$ $C_{6}\|\nabla u\|_{L^{2}\left(\mathbb{R}^{3}\right)}$.

Proof. We have

$$
\int_{\mathbb{R}^{3}} \frac{1+|k|^{2}}{|k|^{2}}|\widehat{\rho}(k)|^{2} d k=\frac{1}{(4 \pi)^{2}} \int_{\mathbb{R}^{3}}|k|^{2}\left(1+|k|^{2}\right)|\widehat{\varphi}(k)|^{2} d k,
$$

and so

$$
\|\varphi\|_{L^{\infty}} \leq \frac{1}{(2 \pi)^{3 / 2}}\|\hat{\varphi}\|_{L^{1}} \leq \frac{1}{(2 \pi)^{3 / 2}}\left(\int_{\mathbb{R}^{3}} \frac{d k}{|k|^{2}\left(1+|k|^{2}\right)}\right)^{1 / 2} 4 \pi\|\rho\|_{\mathfrak{C}}
$$

The rest is easily obtained by the Sobolev inequalities.

Lemma 4. Let $P$ be a projector in $\mathcal{P}_{\Lambda}$ and $Q=P-P^{0}$. Then $D_{Q}$ is bounded.

Proof. Due to the cut-off in Fourier space, $D^{0}$ is bounded on $\mathcal{H}_{\Lambda}$. On the other hand, if $\varphi=\rho * \frac{1}{|.|}$ for some $\rho \in \mathfrak{C}$, then $\varphi \in L^{\infty}$ by Lemma 3 and so this is also a bounded operator. Let us now denote $R(x, y)=\frac{Q(x, y)}{|x-y|}$. We then have

$$
|R f(x)|^{2}=\left|\int_{\mathbb{R}^{3}} \frac{Q(x, y) f(y)}{|x-y|} d y\right|^{2} \leq\left(\int_{\mathbb{R}^{3}} \frac{|Q(x, y)|^{2}}{|x-y|} d y\right) \times\left(\int_{\mathbb{R}^{3}} \frac{|f(y)|^{2}}{|x-y|} d y\right)
$$

and since, by Kato's inequality,

$$
\begin{gathered}
\int_{\mathbb{R}^{3}} \frac{|f(y)|^{2}}{|x-y|} d y \leq \frac{\pi}{2}\left\langle f,\left|D^{0}\right| f\right\rangle \\
\iint_{\mathbb{R}^{6}} \frac{|Q(x, y)|^{2}}{|x-y|} d x d y \leq \frac{\pi}{2} \operatorname{tr}\left(\left|D^{0}\right| Q^{2}\right)
\end{gathered}
$$

this shows that $R \leq C\left|D^{0}\right|^{1 / 2}$ and so $R$ is bounded.

Lemma 5. Let $P$ be a projector in $\mathcal{P}_{\Lambda}$ and $Q=P-P^{0}$. Then $D_{Q} \Gamma \in \mathfrak{S}_{1}^{P_{0}}\left(\mathcal{H}_{\Lambda}\right)$ for all $\Gamma \in \mathcal{G}_{\Lambda}$ and we have

$$
\operatorname{tr}_{P^{0}}\left(D^{0} \Gamma\right)+\alpha D\left(\rho_{Q}-n, \rho_{\Gamma}\right)-\alpha \operatorname{tr}\left(\frac{Q(x, y)}{|x-y|} \Gamma\right)=\operatorname{tr}_{P^{0}}\left(D_{Q} \Gamma\right)
$$

Proof. Remark that $R \Gamma=\frac{Q(x, y)}{|x-y|} \Gamma$ is trace class, since $\frac{Q(x, y)}{|x-y|^{1 / 2}}$ and $\frac{\Gamma(x, y)}{|x-y|^{1 / 2}}$ are in $\mathfrak{S}_{2}$ by (23). Let us now define $D=D^{-\alpha\left(\rho_{Q}-n\right) * \frac{1}{1 \cdot}}=D^{0}+\alpha\left(\rho_{Q}-n\right) * \frac{1}{1 \cdot}$ and $P^{\prime}=\chi_{(-\infty ; 0)}(D)$. By the result of Klaus-Scharf [36] (see also [30] and the 
proof of Theorem [3), it is known that $P^{\prime}-P^{0} \in \mathfrak{S}_{2}\left(\mathcal{H}_{\Lambda}\right)$. Thus $P^{\prime} D \Gamma P^{\prime}=$ $D P^{\prime} \Gamma P^{\prime} \in \mathfrak{S}_{1}\left(\mathcal{H}_{\Lambda}\right)$ since $\Gamma \in \mathfrak{S}_{1}^{P_{0}}=\mathfrak{S}_{1}^{P^{\prime}}$ by Lemma 1, and $D$ is bounded by the proof of Lemma 4. Therefore, $D_{Q} \Gamma=D \Gamma+R \Gamma$ is in $\mathfrak{S}_{1}^{P_{0}}$.

To show the expected equality, we prove

$$
\operatorname{tr}_{P^{0}}\left(D^{0} \Gamma\right)+\alpha D\left(\rho_{Q}^{\prime}, \rho_{\Gamma}\right)=\operatorname{tr}_{P^{0}}(D \Gamma)
$$

where $\rho_{Q}^{\prime}=\rho_{Q}-n \in \mathfrak{C}$. This will end the proof since the other term is trace class. The general idea of the proof is to approximate $\Gamma$ by a trace class operator for which this equality is true, and to pass to the limit. However, the behaviour of the associated density in the space $\mathfrak{C}$ is not obvious and to overcome this difficulty, we shall also approximate the density $\rho_{Q}^{\prime}$ to obtain a potential in $L^{2}\left(\mathbb{R}^{3}\right)$. We thus start by choosing a sequence $\rho_{j}$ which converges as $j \rightarrow+\infty$ to $\rho_{Q}^{\prime}$ in $\mathfrak{C}$, such that $\varphi_{j}=\rho_{j} * \frac{1}{|\cdot|}$ is in $L^{2}\left(\mathbb{R}^{3}\right)$. We can choose for instance $\widehat{\rho_{j}}(k)=\widehat{\rho_{Q}^{\prime}}(k) \chi_{(|k| \geq 1 / j)}$. We now show

$$
\operatorname{tr}_{P^{0}}\left(D^{0} \Gamma\right)+\alpha \int_{\mathbb{R}^{3}} \rho_{\Gamma} \varphi_{j}=\operatorname{tr}_{P^{0}}\left(D_{j} \Gamma\right)
$$

for all $\Gamma \in \mathcal{G}_{\Lambda}$, and where $D_{j}=D^{0}-\alpha \varphi_{j}$. To this end, we may find a sequence $\Gamma_{+-}^{n}$ of finite rank operator which converges to $\Gamma_{+-}=\left(1-P^{0}\right) \Gamma P^{0}$ in $\mathfrak{S}_{2}$. Then

$$
\Gamma^{n}:=\left(\begin{array}{cc}
\Gamma_{++} & \Gamma_{+-}^{n} \\
\left(\Gamma_{+-}^{n}\right)^{*} & \Gamma_{--}
\end{array}\right)
$$

converges to $\Gamma$ in $\mathfrak{S}_{2}$. Since $\Gamma^{n} \in \mathfrak{S}_{1}$ for all $n \geq 0$, we have

$$
\operatorname{tr}_{P^{0}}\left(D^{0} \Gamma^{n}\right)+\alpha \int_{\mathbb{R}^{3}} \rho_{\Gamma^{n}} \varphi_{j}=\operatorname{tr}_{P^{0}}\left(D_{j} \Gamma^{n}\right) .
$$

By (9), the function $Q \in \mathfrak{S}_{2}\left(\mathcal{H}_{\Lambda}\right) \mapsto \rho_{Q} \in L^{2}\left(\mathbb{R}^{3}\right)$ is continuous. Therefore, $\rho_{\Gamma^{n}} \rightarrow \rho_{\Gamma}$ in $L^{2}\left(\mathbb{R}^{3}\right)$. Since $\varphi_{j} \in L^{2}\left(\mathbb{R}^{3}\right)$, we may now pass to the limit in (26) and obtain

$$
\lim _{n \rightarrow \infty}\left(\operatorname{tr}_{P^{0}}\left(D^{0} \Gamma^{n}\right)+\alpha \int_{\mathbb{R}^{3}} \rho_{\Gamma^{n}} \varphi_{j}\right)=\operatorname{tr}_{P^{0}}\left(D^{0} \Gamma\right)+\alpha \int_{\mathbb{R}^{3}} \rho_{\Gamma} \varphi_{j},
$$

where we have used that

$\operatorname{tr}_{P^{0}}\left(D^{0} \Gamma^{n}\right)=\operatorname{tr}\left(D^{0} \Gamma_{++}^{n}\right)+\operatorname{tr}\left(D^{0} \Gamma_{--}^{n}\right)=\operatorname{tr}\left(D^{0} \Gamma_{++}\right)+\operatorname{tr}\left(D^{0} \Gamma_{--}\right)=\operatorname{tr}_{P^{0}}\left(D^{0} \Gamma\right)$.

Let us now pass to the limit in the right hand side. Indeed, we can write, by Lemma 1]

$$
\operatorname{tr}_{P^{0}}\left(D_{j} \Gamma^{n}\right)=\operatorname{tr}_{P_{j}^{\prime}}\left(D_{j} \Gamma^{n}\right)=\operatorname{tr}\left(D_{j} P_{j}^{\prime} \Gamma^{n} P_{j}^{\prime}\right)+\operatorname{tr}\left(D_{j}\left(1-P_{j}^{\prime}\right) \Gamma^{n}\left(1-P_{j}^{\prime}\right)\right)
$$

where $P_{j}^{\prime}=\chi_{(-\infty ; 0)}\left(D_{j}\right)$ and since $P_{j}^{\prime}-P^{0} \in \mathfrak{S}_{2}$ by [36]. Now, using (22), it is easily seen that $P_{j}^{\prime} \Gamma^{n} P_{j}^{\prime} \rightarrow P_{j}^{\prime} \Gamma P_{j}^{\prime}$ and $\left(1-P_{j}^{\prime}\right) \Gamma^{n}\left(1-P_{j}^{\prime}\right) \rightarrow\left(1-P_{j}^{\prime}\right) \Gamma\left(1-P_{j}^{\prime}\right)$ in $\mathfrak{S}_{1}$ as $n \rightarrow \infty$, since this terms can be expanded as a sum of trace class operators and products of at least two Hilbert-Schmidt operators converging 
strongly in $\mathfrak{S}_{2}$. Since $D_{j}$ is bounded by the proof of Lemma 4 , we obtain that $\operatorname{tr}_{P_{j}^{\prime}}\left(D_{j} \Gamma^{n}\right) \rightarrow_{n \rightarrow \infty} \operatorname{tr}_{P_{j}^{\prime}}\left(D_{j} \Gamma\right)=\operatorname{tr}_{P^{0}}\left(D_{j} \Gamma\right)$ by Lemma 1

As a conclusion, we have proved (25) for all $\Gamma \in \mathcal{G}_{\Lambda}$. To finish the proof, it remains to pass to the limit as $j \rightarrow+\infty$. Since

$$
\int_{\mathbb{R}^{3}} \rho_{\Gamma} \varphi_{j}=D\left(\rho_{\Gamma}, \rho_{j}\right)
$$

and $\rho_{\Gamma} \in \mathfrak{C}$ (recall that $\left.\Gamma \in \mathcal{G}_{\Lambda}\right), \rho_{j} \rightarrow \rho_{Q}^{\prime}$ strongly in $\mathfrak{C}$ as $j \rightarrow \infty$, we may pass to the limit in the left hand side of (25). To pass to the limit in the right hand side, we use again the fact that

$$
\operatorname{tr}_{P^{0}}\left(D_{j} \Gamma\right)=\operatorname{tr}_{P_{j}^{\prime}}\left(D_{j} \Gamma\right)=\operatorname{tr}\left(D_{j} P_{j}^{\prime} \Gamma P_{j}^{\prime}\right)+\operatorname{tr}\left(D_{j}\left(1-P_{j}^{\prime}\right) \Gamma\left(1-P_{j}^{\prime}\right)\right) .
$$

By the results of Klaus-Scharf 36] (see also the proof of Theorem 3), it is known that $P_{j}^{\prime}-P^{\prime} \rightarrow 0$ in $\mathfrak{S}_{2}$, since $\rho_{j} \rightarrow \rho_{Q}^{\prime}$ in $\mathfrak{C}$. Using again (22), it is then easily seen that $P_{j}^{\prime} \Gamma P_{j}^{\prime} \rightarrow P^{\prime} \Gamma P^{\prime}$ and $\left(1-P_{j}^{\prime}\right) \Gamma\left(1-P_{j}^{\prime}\right) \rightarrow\left(1-P^{\prime}\right) \Gamma\left(1-P^{\prime}\right)$ in $\mathfrak{S}_{1}$ as $j \rightarrow \infty$. Since $D_{j} \rightarrow D$ in $\mathfrak{S}_{\infty}$ by Lemma 4 we may thus pass to the limit and obtain the desired equality (24).

\subsection{End of the proof of Theorem 2}

We start by proving 1$) \Rightarrow 2$ ). We thus consider a projector $P$ that satisfies the assumption of the Theorem, and is also a solution to the equation $P=$ $\chi_{(-\infty ; 0)}\left(D_{Q}\right)$. We fix some $\Gamma \in \mathcal{G}_{\Lambda}$ and show that $\mathcal{E}(\Gamma) \geq \mathcal{E}(Q)$. To this end, we write $\mathcal{E}(\Gamma)=\mathcal{E}\left(Q+\Gamma^{\prime}\right)$ where $\Gamma^{\prime}=\Gamma-Q=\Gamma+P^{0}-P$. By assumption, $\Gamma$ fulfills $-P^{0} \leq \Gamma \leq 1-P^{0}$, and so $\Gamma^{\prime}$ fulfills $-P \leq \Gamma^{\prime} \leq 1-P$. Using Lemma 5. we may expand $\mathcal{E}\left(Q+\Gamma^{\prime}\right)$ and obtain

$$
\mathcal{E}\left(Q+\Gamma^{\prime}\right)=\operatorname{tr}_{P^{0}}\left(D_{Q} \Gamma^{\prime}\right)+\frac{\alpha}{2} D\left(\rho_{\Gamma^{\prime}}, \rho_{\Gamma^{\prime}}\right)-\frac{\alpha}{2} \iint \frac{\left|\Gamma^{\prime}(x, y)\right|^{2}}{|x-y|} d x d y+\mathcal{E}(Q) .
$$

Using now Lemma 1 we see that it is thus sufficient to prove that

$$
\operatorname{tr}_{P}\left(D_{Q} \Gamma^{\prime}\right)+\frac{\alpha}{2} D\left(\rho_{\Gamma^{\prime}}, \rho_{\Gamma^{\prime}}\right)-\frac{\alpha}{2} \iint \frac{\left|\Gamma^{\prime}(x, y)\right|^{2}}{|x-y|} d x d y>0
$$

for any $\Gamma^{\prime} \in \mathfrak{S}_{1}^{P}\left(\mathcal{H}_{\Lambda}\right)$ such that $\Gamma^{\prime} \neq 0, \rho_{\Gamma^{\prime}} \in \mathfrak{C}$ and $-P \leq \Gamma^{\prime} \leq 1-P$, which is an easy adaptation of the proof of [4, Theorem 2].

We now show 2$) \Rightarrow 1$ ). Let be $P$ which satisfies the assumption of the Theorem, and such that $Q=P-P^{0}$ is a minimizer of $\mathcal{E}$ in $\mathcal{B}_{\Lambda}$. We therefore have, by formula (12),

$$
\operatorname{tr}\left(D_{Q} \gamma\right)+\frac{\alpha}{2} D\left(\rho_{\gamma}, \rho_{\gamma}\right)-\frac{\alpha}{2} \iint \frac{|\gamma(x, y)|^{2}}{|x-y|} d x d y \geq 0
$$

for all $\gamma \in \mathfrak{S}_{1}\left(\mathcal{H}_{\Lambda}\right)$ such that $-P \leq \gamma \leq 1-P$. The proof of Theorem 4 by Bach et al. 4] now implies that $P=\chi_{(-\infty ; 0)}\left(D_{Q}\right)$. Their proof is done with $D^{\alpha \varphi}$ instead of $D_{Q}$ but they also mention that it can be extended to a more general case, provided $0 \notin \sigma\left(D_{Q}\right)$ and $P, 1-P$ leave the domain of $D_{Q}$ invariant, which is the case here. 


\section{Proof of Theorem 3}

In this section, we prove Theorem 3 by using a Banach fixed-point method.

\subsection{Preliminaries}

We start by defining the norms and spaces that will be used to apply this well known result. In fact, one of the main difficulties we faced in this work consisted in finding suitable Banach spaces.

\subsubsection{Norms and spaces}

We choose the following norms

$$
\begin{gathered}
\|Q\|_{\mathcal{Q}}:=\left(\iint E(p-q)^{2} E(p+q)|\widehat{Q}(p, q)|^{2} d p d q\right)^{1 / 2}, \\
\|R\|_{\mathcal{R}}:=\left(\iint \frac{E(p-q)^{2}}{E(p+q)}|\widehat{R}(p, q)|^{2} d p d q\right)^{1 / 2}, \\
\|\rho\|_{\mathfrak{C}}:=\left(\int \frac{E(k)^{2}}{|k|^{2}}|\widehat{\rho}(k)|^{2} d k\right)^{1 / 2}, \\
\|\varphi\|_{\mathcal{Y}}:=\left(\int|k|^{2} E(k)^{2}|\widehat{\varphi}(k)|^{2} d k\right)^{1 / 2},
\end{gathered}
$$

where

$$
E(k)=\sqrt{1+|k|^{2}}
$$

and denote by $\mathcal{Q}, \mathcal{R}, \mathfrak{C}$ and $\mathcal{Y}$ the associated Hilbert spaces. The dual space $\mathfrak{C}^{\prime}$ of $\mathfrak{C}$ will be also useful and we introduce

$$
\|\zeta\|_{\mathfrak{C}^{\prime}}:=\left(\int \frac{|k|^{2}}{E(k)^{2}}|\hat{\zeta}(k)|^{2} d k\right)^{1 / 2}
$$

In the following, it will be easier to use the norm $\|R\|_{\mathcal{R}}$ where $R=\frac{Q(x, y)}{|x-y|}$ in our estimates and a relation with $\|Q\|_{\mathcal{Q}}$ will then be needed. To this end, we first need the following well known Lemma, which will be useful throughout the rest of the proof.

Lemma 6. For all $\xi$ and $\eta$ in $\mathbb{R}^{3}$, we have

$$
\begin{gathered}
\forall s \geq 0, E(\xi)^{s} \leq 2^{\delta(s)}\left(E(\xi-\eta)^{s}+E(\eta)^{s}\right) \\
\forall s \in \mathbb{R}, E(\xi)^{s} \leq 2^{|s|} E(\xi-\eta)^{s} E(\eta)^{|s|},
\end{gathered}
$$

with

$$
\delta(s)=\left\{\begin{array}{lll}
s & \text { if } & 0 \leq s<1 \\
s-1 & \text { if } \quad s \geq 1
\end{array} .\right.
$$


Remark 9. A trivial consequence of (28) is the following inequality

$$
\frac{1}{E(p)+E(q)} \leq \min \left(\frac{1}{E(p+q)}, \frac{1}{E(p-q)}\right)
$$

We shall also need the following

Lemma 7. We have

$$
\sup _{p, q \in \mathbb{R}^{3}} \frac{E(p+q)}{E(p)^{2} E(p-q)^{2}} \leq 2
$$

Proof. Let us introduce the function $f(p, q)=E(p+q) E(p)^{-2} E(p-q)^{-2}$ for $(p, q) \in \mathbb{R}^{3} \times \mathbb{R}^{3}$. We have

$$
0 \leq f(p, q)=\frac{E(2 p-(p-q))}{E(p)^{2} E(p-q)^{2}} \leq \frac{2 E(2 p) E(p-q)}{E(p)^{2} E(p-q)^{2}} \leq \frac{4}{E(p) E(p-q)}
$$

by Lemma [6] Therefore $\lim _{(p, q) \rightarrow \infty} f(p, q)=0$ and $f$ attains its maximum on $\mathbb{R}^{3} \times \mathbb{R}^{3}$. Computing $\nabla_{q} f(p, q)$, we see that at a critical point of $f, p$ and $q$ are always parallel. It therefore suffices to study the function $g(x, y)=$ $E(x+y) E(x)^{-2} E(x-y)^{-2}$ for $(x, y) \in \mathbb{R} \times \mathbb{R}$. It is then easy to see that $\max _{\mathbb{R}^{2}} g<2$ (the bound (31) is indeed not optimal).

Now we can give a connection between $\|R\|_{\mathcal{R}}$ and $\|Q\|_{\mathcal{Q}}$ when $R=\frac{Q(x, y)}{|x-y|}$ (we also recall the easy relation between $\|\rho\|_{\mathfrak{C}}$ and $\|\varphi\|_{\mathcal{Y}}$ when $\varphi=\rho * \frac{1}{|\cdot|}$ ).

Lemma 8. If $\rho \in \mathfrak{C}$ and $Q \in \mathcal{Q}$, then we have $\varphi=\rho * \frac{1}{\mid .} \in \mathcal{Y}$ and $R(x, y)=$ $\frac{Q(x, y)}{|x-y|} \in \mathcal{R}$ and more precisely

$$
\begin{gathered}
\|\varphi\|_{\mathcal{Y}}=4 \pi\|\rho\|_{\mathfrak{C}}, \\
\|R\|_{\mathcal{R}} \leq C_{R}\|Q\|_{\mathcal{Q}}, \\
\left\|R\left|D_{0}\right|^{-1}\right\|_{\mathfrak{S}_{2}} \leq \sqrt{2}\|R\|_{\mathcal{R}},
\end{gathered}
$$

with

$$
C_{R}:=\frac{1}{2 \pi^{2}} \inf _{\theta \in(0 ; 2)} \sup _{x \in \mathbb{R}^{3}}\left(E(2 x)^{\theta} \int_{\mathbb{R}^{3}} \frac{d u}{E(2 u)^{1+\theta}|u-x|^{2}}\right) .
$$

Proof. We have

$$
\widehat{R}(p, q)=\frac{1}{2 \pi^{2}} \int_{\mathbb{R}^{3}} \frac{\widehat{Q}(p-l, q-l)}{|l|^{2}} d l
$$


so we obtain, for some fixed $\theta \in(0 ; 2)$

$$
\begin{aligned}
\|R\|_{\mathcal{R}}^{2} & =\iint \frac{E(p-q)^{2}}{E(p+q)}|\widehat{R}(p, q)|^{2} d p d q \\
& =8 \iint \frac{E(2 v)^{2}}{E(2 u)}|\widehat{R}(u+v, u-v)|^{2} d u d v \\
& =\frac{8}{\left(2 \pi^{2}\right)^{2}} \iiint \int \frac{E(2 v)^{2}}{E(2 u)} \frac{\widehat{Q}(l+v, l-v) \cdot \widehat{Q}\left(l^{\prime}+v, l^{\prime}-v\right)}{|l-u|^{2}\left|l^{\prime}-u\right|^{2}} d u d v d l d l^{\prime} \\
& =\frac{8}{\left(2 \pi^{2}\right)^{2}} \iint d u d v \frac{E(2 v)^{2}}{E(2 u)} \times \\
& \times \iint \frac{E(2 l)^{\frac{1+\theta}{2}} \widehat{Q}(l+v, l-v) E\left(2 l^{\prime}\right)^{\frac{1+\theta}{2}} \widehat{Q}\left(l^{\prime}+v, l^{\prime}-v\right)}{E\left(2 l^{\prime}\right)^{\frac{1+\theta}{2}}|l-u|\left|l^{\prime}-u\right| E(2 l)^{\frac{1+\theta}{2}}|l-u|\left|l^{\prime}-u\right|} d l d l^{\prime} \\
\leq & \frac{8}{\left(2 \pi^{2}\right)^{2}} \iiint \int \frac{E(2 v)^{2}}{E(2 u)} \frac{E(2 l)^{1+\theta}}{E\left(2 l^{\prime}\right)^{1+\theta}} \frac{|\widehat{Q}(l+v, l-v)|^{2}}{|l-u|^{2}\left|l^{\prime}-u\right|^{2}} d u d v d l d l^{\prime} \\
\leq & 8 \iint E(2 v)^{2} E(2 l)|\widehat{Q}(l+v, l-v)|^{2} K_{\theta}(l) d v d l
\end{aligned}
$$

where

$$
K_{\theta}(l):=\frac{E(2 l)^{\theta}}{\left(2 \pi^{2}\right)^{2}} \iint \frac{1}{E(2 u) E\left(2 l^{\prime}\right)^{1+\theta}|l-u|^{2}\left|l^{\prime}-u\right|^{2}} d u d l^{\prime}
$$

Now, let us introduce

$$
C_{\theta}:=\sup _{x \in \mathbb{R}^{3}}\left(E(2 x)^{\theta} \int_{\mathbb{R}^{3}} \frac{d u}{E(2 u)^{1+\theta}|u-x|^{2}}\right) .
$$

Remark that

$$
\int_{\mathbb{R}^{3}} \frac{d u}{E(2 u)^{1+\theta}|u-x|^{2}} \leq \frac{1}{2^{1+\theta}|x|^{\theta}} \int_{\mathbb{R}^{3}} \frac{d u}{|u|^{1+\theta}\left|u-e_{x}\right|^{2}}
$$

where $e_{x}:=x /|x|$, showing that $C_{\theta}<\infty$ when $\theta \in(0 ; 2)$. Now we have

$$
\begin{aligned}
K_{\theta}(l) & =\frac{E(2 l)^{\theta}}{\left(2 \pi^{2}\right)^{2}} \int d u \frac{1}{E(2 u)^{1+\theta}|l-u|^{2}}\left(E(2 u)^{\theta} \int \frac{1}{E\left(2 l^{\prime}\right)^{1+\theta}\left|l^{\prime}-u\right|^{2}} d l^{\prime}\right) \\
& \leq \frac{E(2 l)^{\theta}}{\left(2 \pi^{2}\right)^{2}} \int d u \frac{1}{E(2 u)^{1+\theta}|l-u|^{2}} \times C_{\theta} \leq\left(\frac{C_{\theta}}{2 \pi^{2}}\right)^{2}
\end{aligned}
$$

and so

$$
\|R\|_{\mathcal{R}}^{2} \leq 8\left(\frac{C_{\theta}}{2 \pi^{2}}\right)^{2} \iint E(2 v)^{2} E(2 l)|\widehat{Q}(l+v, l-v)|^{2} d v d l \leq\left(\frac{C_{\theta}}{2 \pi^{2}}\right)^{2}\|Q\|_{\mathcal{Q}}^{2}
$$

which ends the proof of (32).

To prove (33), we remark that we have, by (31),

$$
\left\|R\left|D_{0}\right|^{-1}\right\|_{\mathfrak{S}_{2}}^{2}=\iint \frac{|\widehat{R}(p, q)|^{2}}{E(p)^{2}} d p d q \leq 2 \iint \frac{E(p-q)^{2}|\widehat{R}(p, q)|^{2}}{E(p+q)} d p d q=2\|R\|_{\mathcal{R}}^{2} .
$$




\subsubsection{An estimate from below for $D_{Q}$}

We now state a Lemma in which we give a lower estimate for the operator

$$
D_{Q, \mu}:=D^{0}+\alpha \mu * \frac{1}{|r|}-\alpha \frac{Q(x, y)}{|x-y|}
$$

by $D^{0}$, in terms of the spaces introduced above.

For our result, we are interested in $D_{Q}=D_{Q, \rho_{Q}-n}$ but this definition with an arbitrary density $\mu$ will be useful later on.

Lemma 9. Assume that $(Q, \mu) \in \mathcal{Q} \times \mathfrak{C}$ are such that

$$
\alpha\left(2 \sqrt{\pi}\|\mu\|_{\mathfrak{C}}+\sqrt{2} C_{R}\|Q\|_{\mathcal{Q}}\right)<1 .
$$

Then $D_{Q, \mu}$ is a bounded operator which satisfies

$$
\left|D_{Q, \mu}\right| \geq\left(1-\alpha\left(2 \sqrt{\pi}\|\mu\|_{\mathfrak{C}}+\sqrt{2} C_{R}\|Q\|_{\mathcal{Q}}\right)\right)\left|D^{0}\right|
$$

Proof. We have, with $\varphi^{\prime}=\mu * \frac{1}{|\cdot|}$,

$$
\left\|\varphi^{\prime} u\right\|_{L^{2}} \leq\left\|\varphi^{\prime}\right\|_{L^{\infty}}\|u\|_{L^{2}} \leq 2 \pi^{1 / 2}\|\mu\|_{\mathfrak{C}}\left\|\left|D_{0}\right| \cdot u\right\|_{L^{2}}
$$

by Lemma 3, and

$\|R u\|_{L^{2}}=\left\|R\left|D_{0}\right|^{-1}\left|D_{0}\right| u\right\|_{L^{2}} \leq\left\|R\left|D_{0}\right|^{-1}\right\|_{\mathfrak{S}_{2}}\left\|\left|D_{0}\right| u\right\|_{L^{2}} \leq \sqrt{2} C_{R}\|Q\|_{\mathcal{Q}}\left\|\left|D_{0}\right| \cdot u\right\|_{L^{2}}$

by (33). This shows that $\left|\varphi^{\prime}-R\right| \leq\left(2 \pi^{1 / 2}\|\mu\|_{\mathfrak{C}}+\sqrt{2} C_{R}\|Q\|_{\mathcal{Q}}\right)\left|D^{0}\right|$, the square root being monotone. This proves that $D_{Q, \mu}$ is bounded since $D_{0}$ is bounded on $\mathcal{H}_{\Lambda}$, and gives the expected inequality.

Remark that Lemma 9 will be useful when we shall apply Theorem 2 (see the condition (14) in the statement). It also implies $0 \notin \sigma\left(D_{Q, \mu}\right)$, a fact that will be used to compute the projection $\chi_{(-\infty ; 0)}\left(D_{Q, \mu}\right)$.

\subsubsection{Expansion by Cauchy's formula}

We want to solve the equation

$$
Q=\chi_{(-\infty ; 0)}\left(D^{0}+\alpha\left(\rho_{Q}-n\right) * \frac{1}{|\cdot|}-\alpha \frac{Q(x, y)}{|x-y|}\right)-\chi_{(-\infty ; 0)}\left(D^{0}\right):=F_{1}(Q) .
$$

If $\alpha\left(2 \sqrt{\pi}\left\|\rho_{Q}-n\right\|_{\mathfrak{C}}+\sqrt{2} C_{R}\|Q\|_{\mathcal{Q}}\right)<1$, then $0 \notin \sigma\left(D_{Q}\right)$ by Lemma 9 , We may thus use the method of [29] and expand $F_{1}$ by Cauchy's formula

$$
F_{1}(Q)=-\frac{1}{2 \pi} \int_{-\infty}^{+\infty} d \eta\left(\frac{1}{D_{Q}+i \eta}-\frac{1}{D^{0}+i \eta}\right)=\sum_{n=1}^{\infty} \alpha^{n} Q_{n}
$$

where

$$
Q_{n}=-\frac{1}{2 \pi} \int_{-\infty}^{+\infty} d \eta \frac{1}{D^{0}+i \eta}\left(\left(R_{Q}-\varphi_{Q}^{\prime}\right) \frac{1}{D^{0}+i \eta}\right)^{n}
$$




$$
\rho_{Q}^{\prime}=\rho_{Q}-n, \quad \varphi_{Q}^{\prime}=\rho_{Q}^{\prime} * \frac{1}{|r|}, \quad R_{Q}(x, y)=\frac{Q(x, y)}{|x-y|} .
$$

We shall write

$$
\begin{gathered}
Q_{n}=\sum_{k, l / k+l=n} Q_{k, l} \\
Q_{k, l}=\frac{(-1)^{l+1}}{2 \pi} \sum_{\substack{I \cup J=\{1, \ldots, n\},|I|=k,|J|=l}} \int_{-\infty}^{+\infty} d \eta \frac{1}{D^{0}+i \eta} \prod_{j=1}^{n}\left(R_{j} \frac{1}{D^{0}+i \eta}\right)
\end{gathered}
$$

where $R_{j}=R_{Q}$ if $j \in I$ and $R_{j}=\varphi_{Q}^{\prime}$ if $j \in J$ ( $Q_{k, l}$ is the sum of all the terms containing $k R_{Q}$ 's and $l \varphi_{Q}^{\prime}$ 's). We also denote $\rho_{k, l}:=\rho_{Q_{k, l}}$.

Hence our equation can be written

$$
\left\{\begin{array}{l}
Q=\sum_{n=1}^{\infty} \alpha^{n} Q_{n}\left(Q, \rho_{Q}^{\prime}\right) \\
\rho_{Q}=\sum_{n=1}^{\infty} \alpha^{n} \rho_{n}\left(Q, \rho_{Q}^{\prime}\right),
\end{array}\right.
$$

where we recall that $Q_{n}$ and $\rho_{n}$ depend on both $Q$ and $\rho_{Q}^{\prime}=\rho_{Q}-n$. In order to have a better condition on $\alpha$ and $\Lambda$, we shall now change the second equation for the density, by taking into account the special form of the first order term $\rho_{1}$. To this end, we need to compute this term explicitely.

\subsubsection{The first order density}

Recall that

$$
Q_{0,1}=\frac{1}{2 \pi} \int_{-\infty}^{+\infty} d \eta \frac{1}{D^{0}+i \eta} \varphi_{Q}^{\prime} \frac{1}{D^{0}+i \eta}
$$

so that

$$
\widehat{Q_{0,1}}(p, q)=(2 \pi)^{-5 / 2} \int_{-\infty}^{+\infty} d \eta \frac{1}{\boldsymbol{\alpha} \cdot p+\beta+i \eta} \widehat{\varphi_{Q}^{\prime}}(p-q) \frac{1}{\boldsymbol{\alpha} \cdot q+\beta+i \eta}
$$

We now introduce

$$
\begin{aligned}
M(p, q) & :=\frac{1}{\pi} \int_{-\infty}^{+\infty} d \eta \frac{1}{\boldsymbol{\alpha} \cdot p+\beta+i \eta} \cdot \frac{1}{\boldsymbol{\alpha} \cdot q+\beta+i \eta} \\
& =\frac{1}{\pi} \int_{-\infty}^{+\infty} d \eta \frac{\boldsymbol{\alpha} \cdot p+\beta-i \eta}{p^{2}+1+\eta^{2}} \cdot \frac{\boldsymbol{\alpha} \cdot q+\beta-i \eta}{q^{2}+1+\eta^{2}} \\
& =\frac{1}{E(p)+E(q)}\left(\frac{(\boldsymbol{\alpha} \cdot p+\beta)}{E(p)} \frac{(\boldsymbol{\alpha} \cdot q+\beta)}{E(q)}-1\right)
\end{aligned}
$$

Hence

$$
\widehat{Q_{0,1}}(p, q)=\frac{1}{2^{5 / 2} \pi^{3 / 2}} \widehat{\varphi_{Q}^{\prime}}(p-q) M(p, q)
$$


This enables us to compute

$$
\begin{aligned}
\widehat{\rho_{0,1}}(k) & =\frac{1}{(2 \pi)^{3 / 2}} \int_{|l| \leq \Lambda} \operatorname{Tr}_{\mathbb{C}^{4}}\left(\widehat{Q_{0,1}}(l+k / 2, l-k / 2)\right) d l \\
& =\frac{1}{16 \pi^{3}} \widehat{\varphi_{Q}^{\prime}}(k) \int_{|l| \leq \Lambda} \operatorname{Tr}_{\mathbb{C}^{4}}(M(l+k / 2, l-k / 2)) d l \\
& =-\frac{1}{4 \pi} \widehat{\varphi_{Q}^{\prime}}(k)|k|^{2} B_{\Lambda}(k) \\
& =-\widehat{\rho_{Q}^{\prime}}(k) B_{\Lambda}(k)
\end{aligned}
$$

where

$$
B_{\Lambda}(k)=-\frac{1}{\pi^{2}|k|^{2}} \int_{|l| \leq \Lambda} \frac{(l+k / 2) \cdot(l-k / 2)+1-E(l+k / 2) E(l-k / 2)}{E(l+k / 2) E(l-k / 2)(E(l+k / 2)+E(l-k / 2))} d l .
$$

This function is computed in 45

$$
B_{\Lambda}(k)=\frac{1}{\pi} \int_{0}^{\frac{\Lambda}{E(\Lambda)}} \frac{z^{2}-z^{4} / 3}{1-z^{2}} \frac{d z}{1+|k|^{2}\left(1-z^{2}\right) / 4}
$$

and it is logarithmically divergent since

$$
B_{\Lambda}(0)=\frac{1}{\pi} \int_{0}^{\frac{\Lambda}{E(\Lambda)}} \frac{z^{2}-z^{4} / 3}{1-z^{2}} d z=\frac{2}{3 \pi} \log (\Lambda)-\frac{5}{9 \pi}+\frac{2}{3 \pi} \log 2+O\left(1 / \Lambda^{2}\right) .
$$

\subsubsection{Equation}

We are now able to introduce the function on which we shall apply the fixedpoint theorem. According to what we said above, the equation in $\rho_{Q}$ can be written

$$
\rho_{Q}=\sum_{n=1}^{\infty} \alpha^{n} \rho_{n}\left(Q, \rho_{Q}^{\prime}\right)
$$

or equivalently (we forget the dependence in $Q$ and $\rho_{Q}^{\prime}$ for simplicity)

$$
\widehat{\rho_{Q}}(k)=-\alpha B_{\Lambda}(k) \widehat{\rho_{Q}^{\prime}}(k)+\alpha \widehat{\rho_{1,0}}(k)+\sum_{n=2}^{\infty} \alpha^{n} \widehat{\rho_{n}}(k)
$$

and

$$
\widehat{\rho_{Q}^{\prime}}(k)=-\frac{1}{1+\alpha B_{\Lambda}(k)} \widehat{n}(k)+\frac{1}{1+\alpha B_{\Lambda}(k)}\left(\alpha \widehat{\rho_{1,0}}(k)+\sum_{n=2}^{\infty} \alpha^{n} \widehat{\rho_{n}}(k)\right),
$$

which is more adapted to a fixed-point argument, since the divergent term $B_{\Lambda}(k)$ appears now in the denominator.

Notice that we now study an equation for the full density $\rho_{Q}^{\prime}=\rho_{Q}-n$ and not for $\rho_{Q}$ as previously. We therefore introduce the following space:

$$
\mathcal{X}=\mathcal{Q} \times \mathfrak{C}
$$


consisting of all the pairs $\left(Q, \rho^{\prime}\right)$ such that $Q \in \mathcal{Q}$ and $\rho^{\prime} \in \mathfrak{C}$. Notice that in this space, $\rho^{\prime}$ can be different from $\rho_{Q}-n$. However, we shall find a solution of the equations in this space, which satisfies $\rho^{\prime}=\rho_{Q}-n$. We also introduce on $\mathcal{X}$ the norm

$$
\left\|\left(Q, \rho^{\prime}\right)\right\|=C_{R} \sqrt{2}\|Q\|_{\mathcal{Q}}+2 \sqrt{\pi}\left\|\rho^{\prime}\right\|_{\mathfrak{C}},
$$

where we recall that $C_{R}$ is defined in Lemma 8 . In the following, we shall keep the notation $\rho^{\prime}$ to remind the reader that the equation indeed concerns $\rho_{Q}^{\prime}$ and not $\rho_{Q}$.

We now introduce the function $F: \mathcal{X} \rightarrow \mathcal{X}$ defined by

$$
F\left(Q, \rho^{\prime}\right)=\left(F_{Q}\left(Q, \rho^{\prime}\right), F_{\rho}\left(Q, \rho^{\prime}\right)\right)
$$

where

$$
\begin{gathered}
F_{Q}\left(Q, \rho^{\prime}\right)=\chi_{(-\infty ; 0)}\left(D_{Q, \rho^{\prime}}\right)-P^{0}=\sum_{n=1}^{\infty} \alpha^{n} Q_{n}\left(Q, \rho^{\prime}\right) \\
\left.\widehat{F_{\rho}\left(Q, \rho^{\prime}\right.}\right)=-\frac{1}{1+\alpha B_{\Lambda}} \widehat{n}+\frac{1}{1+\alpha B_{\Lambda}}\left(\alpha \widehat{\rho_{1,0}}\left(Q, \rho^{\prime}\right)+\sum_{n=2}^{\infty} \alpha^{n} \widehat{\rho_{n}}\left(Q, \rho^{\prime}\right)\right),
\end{gathered}
$$

$Q_{n}\left(Q, \rho^{\prime}\right)$ and $\rho_{n}\left(Q, \rho^{\prime}\right)$ being defined in (37) (replace $\rho_{Q}^{\prime}$ by $\left.\rho^{\prime}\right)$. Remark that $\rho_{n}=\rho_{Q_{n}}$ for all $n \geq 2$. In the proof of Theorem 3] we solve the fixed-point equation in $\mathcal{X}$

$$
F\left(Q, \rho^{\prime}\right)=\left(Q, \rho^{\prime}\right)
$$

\subsection{Existence of a fixed-point of $F$}

To prove our main Theorem, we need the following estimates

Proposition 10. Assume that $\left(Q, \rho^{\prime}\right) \in \mathcal{X}$ is such that $0 \notin \sigma\left(D_{Q, \rho^{\prime}}\right)$. Then we have

$$
\begin{gathered}
\left\|F\left(Q, \rho^{\prime}\right)\right\| \leq 2 \sqrt{\pi}\|n\|_{\mathfrak{C}}+\kappa_{1}(\Lambda) \alpha\left\|\left(Q, \rho^{\prime}\right)\right\|+\sum_{n=2}^{+\infty} \kappa_{n}\left(\alpha\left\|\left(Q, \rho^{\prime}\right)\right\|\right)^{n} \\
\left\|F^{\prime}\left(Q, \rho^{\prime}\right)\right\| \leq \kappa_{1}(\Lambda) \alpha+\alpha \sum_{n=2}^{+\infty} n \kappa_{n}\left(\alpha\left\|\left(Q, \rho^{\prime}\right)\right\|\right)^{n-1}
\end{gathered}
$$

where

$$
\kappa_{1}(\Lambda)=\max \left(\frac{C_{R} \sqrt{2}}{\sqrt{\pi}} \sqrt{\log \Lambda}, \sqrt{2} C_{R}+\frac{\sqrt{\log \Lambda}}{2^{3 / 2} \sqrt{\pi}}\right) \sim_{\Lambda \rightarrow \infty} \frac{C_{R} \sqrt{2}}{\sqrt{\pi}} \sqrt{\log \Lambda}
$$

and $\left(\kappa_{n}\right)_{n \geq 2}$ is a sequence of positive numbers independent of $\Lambda$ and which satisfies $\kappa_{n} \sim_{n \rightarrow \infty} K \sqrt{n}$ for some constant $K$. 
To prove this proposition, we have to do some tedious estimates. Before starting this proof, let us show that Theorem 3 follows from Proposition 10.

Proof of Theorem [3. We introduce the function $f(x)=\sum_{n=2}^{\infty} \kappa_{n} x^{n}$, which is a power series with a radius of convergence equal to 1 . The estimates (45) and (46) can be written

$$
\begin{gathered}
\left\|F\left(Q, \rho^{\prime}\right)\right\| \leq 2 \sqrt{\pi}\|n\|_{\mathfrak{C}}+\kappa_{1}(\Lambda) \alpha\left\|\left(Q, \rho^{\prime}\right)\right\|+f\left(\alpha\left\|\left(Q, \rho^{\prime}\right)\right\|\right) \\
\left\|F^{\prime}\left(Q, \rho^{\prime}\right)\right\| \leq \kappa_{1}(\Lambda) \alpha+\alpha f^{\prime}\left(\alpha\left\|\left(Q, \rho^{\prime}\right)\right\|\right) .
\end{gathered}
$$

To apply the Banach fixed-point theorem, we now have to find a ball $B(0, R) \subset$ $\mathcal{X}$ which is invariant under the function $F$ and on which $F$ is a contraction. Let $R>0$ be some fixed radius. We have

$$
\sup _{\left(Q, \rho^{\prime}\right) \in B(0, R)}\left\|F^{\prime}\left(Q, \rho^{\prime}\right)\right\| \leq \kappa_{1}(\Lambda) \alpha+\alpha f^{\prime}(\alpha R):=\mu .
$$

Moreover, we also have

$$
\left\|F\left(Q, \rho^{\prime}\right)\right\| \leq\left\|F\left(Q, \rho^{\prime}\right)-F(0,0)\right\|+\|F(0,0)\| \leq \mu\left\|\left(Q, \rho^{\prime}\right)\right\|+\|F(0,0)\| .
$$

Therefore a condition for the ball $B(0, R)$ to be invariant under the action of $F$ is $\|F(0,0)\| \leq(1-\mu) R$. Notice that since $F(0,0) \neq(0,0)$, this inequality also contains the contraction condition $\mu<1$. Additionally due to Lemma 9 we assume $\alpha R<1$ as well as

$$
\frac{\alpha \pi}{4(1-\alpha R)} \leq 1
$$

due to Theorem 2 and Lemma 9 which is equivalent to

$$
\alpha \leq \frac{1}{\pi / 4+R}
$$

As a conclusion, if $(\alpha, R)$ fulfills

$$
\left\{\begin{array}{l}
2 \sqrt{\pi}\|n\|_{\mathfrak{C}}+\alpha R \kappa_{1}(\Lambda)+\alpha R f^{\prime}(\alpha R) \leq R \\
\alpha \leq \frac{1}{\pi / 4+R}
\end{array}\right.
$$

then we are able to apply the Banach Fixed-Point Theorem on $B(0, R)$. Remark that these inequalities also contain the conditions $\mu<1$ and $\alpha R<1$. Notice also that if $(\alpha, R)$ is a solution to (48), then $\left(\alpha^{\prime}, R\right)$ is a solution to (48) for all $\alpha^{\prime} \leq \alpha$, since the function which appears on the left of (48) is increasing in $\alpha$.

Now, if we assume that $2 \sqrt{\pi} \alpha\|n\|_{\mathfrak{C}} \leq b$, we obtain that if $(\alpha, R)$ fulfills

$$
\left\{\begin{array}{l}
\frac{b}{\alpha}+\alpha R \kappa_{1}(\Lambda)+\alpha R f^{\prime}(\alpha R) \leq R \\
\alpha \leq \frac{1}{\pi / 4+R},
\end{array}\right.
$$


then it also fulfills (48). The first inequation of (49) is simpler when it is written in terms of the variables $\alpha$ and $x:=\alpha R$. It becomes

$$
\frac{b}{\alpha}+\kappa_{1}(\Lambda) x+x f^{\prime}(x) \leq \frac{x}{\alpha}
$$

which implies $x \in[b ; 1)$. Now, given $b, \Lambda$ and $x$ let us call $a_{b, \Lambda}(x)$ the maximal value of $\alpha$ such that (50) holds, i.e.

$$
a_{b, \Lambda}(x)=\frac{x-b}{\kappa_{1}(\Lambda) x+x f^{\prime}(x)}
$$

which is defined for $x$ in $[b ; 1)$. Since $\lim _{x \rightarrow 1} a_{b, \Lambda}(x)=a_{b, \Lambda}(b)=0$, we may denote by $x_{\Lambda} \in(b ; 1)$ the largest maximizer of the function $a_{b, \Lambda}$ in the interval $[b ; 1)$.

We now define $R_{b}(\Lambda):=x_{\Lambda} / a_{b, \Lambda}\left(x_{\Lambda}\right)$ and

$$
\alpha_{b}(\Lambda):=\min \left(a_{b, \Lambda}\left(x_{\Lambda}\right), \frac{1}{\pi / 4+R_{b}(\Lambda)}\right) .
$$

As a conclusion, for all $0 \leq \alpha \leq \alpha_{b}(\Lambda),\left(\alpha, R_{b}(\Lambda)\right)$ is a solution of (48). This means that $F$ is a contraction on $B\left(0, R_{b}(\Lambda)\right)$, on which we can apply Banach Theorem. This gives a unique solution to the equation $F\left(Q, \rho^{\prime}\right)=\left(Q, \rho^{\prime}\right)$ in $B\left(0, R_{b}(\Lambda)\right) \subset \mathcal{X}$.

Let us now show that $P$ is indeed a solution to (6). In fact $\rho^{\prime}$ is a solution to (42) and so $\rho=\rho^{\prime}+n$ is a solution to (41). On the other hand, we have $Q=\chi_{(-\infty ; 0)}\left(D_{Q, \rho^{\prime}}\right)-P^{0}$, and (41) means exactly that $\rho=\rho_{Q}$. Hence, $P$ is a solution to $P=\chi_{(-\infty ; 0)}\left(D_{Q}\right)$. Thanks to the proof, we know that $P$ satisfies the assumptions of Theorem 2 and so $P$ is the unique BDF-stable vacuum (i.e. $P-P^{0}$ is the unique global minimizer of the BDF energy).

To end the proof, let us study the behaviour of $\alpha_{b}(\Lambda)$ as $\Lambda \rightarrow \infty$. Computing $\mathrm{d} a_{b, \Lambda}(x) / \mathrm{d} x$, we find that $x_{\Lambda}$ must satisfy the equation

$$
\kappa_{1}(\Lambda)+f^{\prime}\left(x_{\Lambda}\right)=\frac{x_{\Lambda}\left(x_{\Lambda}-b\right)}{b} f^{\prime \prime}\left(x_{\Lambda}\right)
$$

Since $\kappa_{1}(\Lambda)$ diverges as $\Lambda \rightarrow \infty$, we see that $f^{\prime \prime}\left(x_{\Lambda}\right) \rightarrow \infty$ and therefore $x_{\Lambda} \rightarrow$ 1 as $\Lambda \rightarrow \infty$. Now, since $f^{\prime}(x)=o_{x \rightarrow 1}\left(f^{\prime \prime}(x)\right)$, we obtain that $f^{\prime}\left(x_{\Lambda}\right)=$ $o_{\Lambda \rightarrow \infty}\left(\kappa_{1}(\Lambda)\right)$. Thus

$$
\alpha_{b, \Lambda}\left(x_{\Lambda}\right) \sim_{\Lambda \rightarrow \infty} \frac{1-b}{\kappa_{1}(\Lambda)} \quad \text { and } \quad R_{b}(\Lambda) \sim_{\Lambda \rightarrow \infty} \frac{\kappa_{1}(\Lambda)}{1-b} .
$$

As a conclusion,

$$
\alpha_{b}(\Lambda) \sim_{\Lambda \rightarrow \infty} \frac{1-b}{\kappa_{1}(\Lambda)} \sim_{\Lambda \rightarrow \infty} \frac{\sqrt{\pi}(1-b)}{C_{R} \sqrt{2} \sqrt{\log \Lambda}}=\frac{C(1-b)}{\sqrt{\log \Lambda}}
$$

with $C=\frac{\sqrt{\pi}}{\sqrt{2} C_{R}}$. 


\subsection{Proof of Proposition 10; estimates}

In this section, we prove the claimed estimates of Proposition 10. We will have to introduce many constants. For the sake of clarity, a guide is provided to the reader at the very end of the proof, section 4.3.5.

Remark first that we have

$$
\frac{1}{1+\alpha B_{\Lambda}(k)} \leq 1
$$

for all $k \in \mathbb{R}^{3}$. Therefore, to estimate the norm $\left\|F_{\rho}\left(Q, \rho^{\prime}\right)\right\|_{\mathfrak{C}}$, it suffices to estimate the norms of $\rho_{1,0}\left(Q, \rho^{\prime}\right)$ and $\rho_{n}\left(Q, \rho^{\prime}\right)$, due to (44).

For $\left(Q, \rho^{\prime}\right) \in \mathcal{X}$, we introduce the notation $R=\frac{Q(x, y)}{|x-y|} \in \mathcal{R}$, and $\varphi^{\prime}=\rho^{\prime} * \frac{1}{\mid \cdot}$. We then remark that

$$
\left\|F\left(Q, \rho^{\prime}\right)\right\| \leq 2 \sqrt{\pi}\|n\|_{\mathfrak{C}}+\alpha\left\|\left(Q_{1}, \rho_{1,0}\right)\right\|+\sum_{n \geq 2} \alpha^{n}\left\|\left(Q_{n}, \rho_{n}\right)\right\|
$$

and estimate each term separately. A similar argument can be done for $F^{\prime}\left(Q, \rho^{\prime}\right)$.

\subsubsection{First order terms}

Lemma 11. We have the following estimates:

$$
\begin{gathered}
\left\|Q_{0,1}\right\|_{\mathcal{Q}} \leq \frac{(\log \Lambda)^{1 / 2}}{2 \pi}\left\|\varphi^{\prime}\right\|_{\mathcal{Y}}=2(\log \Lambda)^{1 / 2}\left\|\rho^{\prime}\right\|_{\mathfrak{C}}, \\
\left\|Q_{1,0}\right\|_{\mathcal{Q}} \leq\left\|R_{Q}\right\|_{\mathcal{R}} \leq C_{R}\|Q\|_{\mathcal{Q}}, \quad\left\|\rho_{1,0}\right\|_{\mathfrak{C}} \leq \frac{C_{R}(\log \Lambda)^{1 / 2}}{4 \pi}\|Q\|_{\mathcal{Q}} .
\end{gathered}
$$

Therefore

$$
\left\|\left(Q_{1}, \rho_{1,0}\right)\right\| \leq \kappa_{1}(\Lambda)\left\|\left(Q, \rho^{\prime}\right)\right\|
$$

where

$$
\kappa_{1}(\Lambda)=\max \left(\frac{C_{R} \sqrt{2}}{\sqrt{\pi}} \sqrt{\log \Lambda}, \sqrt{2} C_{R}+\frac{\sqrt{\log \Lambda}}{2^{3 / 2} \sqrt{\pi}}\right) .
$$

Proof. Recall that

$$
\widehat{Q_{0,1}}(p, q)=\frac{1}{2^{5 / 2} \pi^{3 / 2}} \widehat{\varphi^{\prime}}(p-q) M(p, q)
$$

where the matrix $M(p, q)$ is defined in (38), and whose properties are summarized in the following

Lemma 12. Let $\Lambda^{+}(p)=\frac{\boldsymbol{\alpha} \cdot p+\beta+E(p)}{2 E(p)}$ and $\Lambda^{-}(p)=\frac{-(\boldsymbol{\alpha} \cdot p+\beta)+E(p)}{2 E(p)}$ be the projections matrices in $\mathbb{C}^{4}$ onto the eigenspaces of $D^{0}$ in Fourier space. We then have

$$
\begin{gathered}
\operatorname{Tr}_{\mathbb{C}^{4}}(M(p, q))=-4 \frac{1}{E(p)+E(q)} \operatorname{Tr}_{\mathbb{C}^{4}}\left(\Lambda^{+}(p) \Lambda^{-}(q)\right) \\
|M(p, q)|^{2}=\operatorname{Tr}_{\mathbb{C}^{4}}\left(M(p, q) M(p, q)^{*}\right)=8 \frac{1}{(E(p)+E(q))^{2}} \operatorname{Tr}_{\mathbb{C}^{4}}\left(\Lambda^{+}(p) \Lambda^{-}(q)\right)
\end{gathered}
$$




$$
\operatorname{Tr}_{\mathbb{C}^{4}}\left(\Lambda^{+}(p) \Lambda^{-}(q)\right)=\operatorname{Tr}_{\mathbb{C}^{4}}\left(\Lambda^{-}(p) \Lambda^{+}(q)\right)=1-\frac{p \cdot q+1}{E(p) E(q)} .
$$

Moreover, we have

$$
\forall p, q \in \mathbb{R}^{3}, \quad \operatorname{Tr}_{\mathbb{C}^{4}}\left(\Lambda^{+}(p) \Lambda^{-}(q)\right) \leq \min \left(\frac{|p-q|^{2}}{2 E((p+q) / 2)^{2}}, 2\right) .
$$

Proof of Lemma 12. We only prove (54). We have $\operatorname{Tr}_{\mathbb{C}^{4}}\left(\Lambda^{+}(p) \Lambda^{-}(q)\right) \leq$ $\left|\Lambda^{+}(p)\right|\left|\Lambda^{-}(q)\right|=2$, so when $t:=\frac{|p-q|^{2}}{4 E((p+q) / 2)^{2}} \geq 1$, there is nothing to prove. Now we have

$$
\begin{aligned}
1-\frac{p \cdot q+1}{E(p) E(q)} & =1-\frac{l^{2}-k^{2}+1}{E(l+k) E(l-k)} \text { with } l=\frac{p+q}{2}, k=\frac{p-q}{2} \\
& =1-\frac{1-t}{\sqrt{(1+t)^{2}-4 z t}}
\end{aligned}
$$

where $t=|k|^{2} / E(l)^{2}$ and $z=\frac{(l \cdot k)^{2}}{|k|^{2}\left(1+l^{2}\right)} \in[0 ; 1)$. When $t \in[0 ; 1)$ and $z \in[0 ; 1)$, the expression above is decreasing in $z$ and so we obtain

$$
1-\frac{p \cdot q+1}{E(p) E(q)} \leq 1-\frac{1-t}{\sqrt{(1+t)^{2}}}=\frac{2 t}{1+t} \leq 2 t
$$

which ends the proof.

- Let us now treat $Q_{0,1}$. From (53), we obtain

$$
\left|\widehat{Q_{0,1}}(p, q)\right|^{2}=\frac{1}{2^{5} \pi^{3}}\left|\widehat{\varphi}^{\prime}\right|^{2}(p-q)|M(p, q)|^{2}
$$

and so

$$
\begin{aligned}
& \iint E(p-q)^{2} E(p+q)\left|\widehat{Q_{0,1}}(p, q)\right|^{2} d p d q \\
& =\frac{1}{2^{5} \pi^{3}} \iint d k d u E(k)^{2} E(2 u)\left|\widehat{\varphi^{\prime}}\right|^{2}(k) \chi(|u| \leq \Lambda)|M(u+k / 2, u-k / 2)|^{2} \\
& \quad \leq \frac{4}{2^{5} \pi^{3}}\left(\int d k|k|^{2} E(k)^{2}\left|\widehat{\varphi^{\prime}}\right|^{2}(k)\right)\left(\int_{|u| \leq \Lambda} \frac{1}{E(2 u) E(u)^{2}}\right)
\end{aligned}
$$

by Lemma 12. Now we have

$$
\int_{|u| \leq \Lambda} \frac{d u}{E(2 u) E(u)^{2}}=4 \pi\left(\frac{1}{2} \operatorname{argsh}(2 \Lambda)+\frac{1}{\sqrt{3}} \operatorname{argth}\left(\frac{\sqrt{3} \Lambda}{\sqrt{1+4 \sqrt{\Lambda}}}\right)\right) \leq 2 \pi \log \Lambda
$$

for $\Lambda \geq 3$. So we obtain

$$
\left\|Q_{0,1}\right\|_{\mathcal{Q}} \leq \frac{(\log \Lambda)^{1 / 2}}{2 \pi}\left\|\varphi^{\prime}\right\|_{\mathcal{Y}}=2(\log \Lambda)^{1 / 2}\left\|\rho^{\prime}\right\|_{\mathfrak{C}}
$$

- $\rho_{1,0}$ and $Q_{1,0}$. We have

$$
Q_{1,0}=-\frac{1}{2 \pi} \int_{-\infty}^{+\infty} d \eta \frac{1}{D^{0}+i \eta} R \frac{1}{D^{0}+i \eta}
$$


so that

$$
\begin{aligned}
\widehat{Q_{1,0}}(p, q) & =-(2 \pi)^{-1} \int_{-\infty}^{+\infty} d \eta \frac{1}{\boldsymbol{\alpha} \cdot p+\beta+i \eta} \widehat{R}(p, q) \frac{1}{\boldsymbol{\alpha} \cdot q+\beta+i \eta} \\
& =-\frac{1}{2} \frac{1}{E(p)+E(q)}\left(\frac{(\boldsymbol{\alpha} \cdot p+\beta)}{E(p)} \widehat{R}(p, q) \frac{(\boldsymbol{\alpha} \cdot q+\beta)}{E(q)}-\widehat{R}(p, q)\right)
\end{aligned}
$$

and

$$
\left|\widehat{Q_{1,0}}(p, q)\right|^{2} \leq \frac{1}{(E(p)+E(q))^{2}}|\widehat{R}(p, q)|^{2} \leq \frac{1}{E(p+q)^{2}}|\widehat{R}(p, q)|^{2},
$$

showing that

$$
\left\|Q_{1,0}\right\|_{\mathcal{Q}} \leq\|R\|_{\mathcal{R}} \leq C_{R}\|Q\|_{\mathcal{Q}}
$$

Now, we have

$$
\begin{aligned}
\widehat{\rho_{1,0}}(k) & =\frac{1}{(2 \pi)^{3 / 2}} \int_{\mathbb{R}^{3}} \operatorname{Tr}_{\mathbb{C}^{4}}\left(\widehat{Q_{1,0}}\left(l+\frac{k}{2}, l-\frac{k}{2}\right)\right) d l \\
& =-\frac{1}{2^{5 / 2} \pi^{3 / 2}} \int_{\mathbb{R}^{3}} \operatorname{Tr}_{\mathbb{C}^{4}}\left(\widehat{R}\left(l+\frac{k}{2}, l-\frac{k}{2}\right) M\left(l+\frac{k}{2}, l-\frac{k}{2}\right)\right) \chi(|l| \leq \Lambda) d l,
\end{aligned}
$$

so we obtain

$$
\begin{aligned}
\left|\widehat{\rho_{1,0}}(k)\right| \leq \frac{1}{2^{5 / 2} \pi^{3 / 2}}\left(\int_{\mathbb{R}^{3}} E(2 l)^{-1}\right. & \left.|\widehat{R}(l+k / 2, l-k / 2)|^{2} d l\right)^{1 / 2} \times \\
& \times\left(\int_{\mathbb{R}^{3}} E(2 l)|M(l+k / 2, l-k / 2)|^{2} d l\right)^{1 / 2}
\end{aligned}
$$

and finally

$$
\int \frac{E(k)^{2}}{|k|^{2}}\left|\widehat{\rho_{1,0}}(k)\right|^{2} d k \leq \frac{1}{2^{5} \pi^{3}}\|R\|_{\mathcal{R}}^{2} \int_{|l| \leq \Lambda} \frac{1}{E(2 l) E(l)^{2}} d l \leq \frac{\log \Lambda}{2^{4} \pi^{2}}\|R\|_{\mathcal{R}}^{2}
$$

by (55) which implies

$$
\left\|\rho_{1,0}\right\|_{\mathfrak{C}} \leq \frac{C_{R}(\log \Lambda)^{1 / 2}}{4 \pi}\|Q\|_{\mathcal{Q}}
$$

\subsubsection{Second order terms}

To simplify the presentation, we introduce the following notation:

$$
S_{p, q}:=(4 \pi)(2 \pi)^{-3 / p}\left(\int_{\mathbb{R}^{3}} \frac{d u}{E(u)^{q}}\right)^{\frac{1}{p}}, \quad S_{p}:=S_{p, p}, \quad K_{p}:=\frac{1}{2 \pi} \int_{-\infty}^{+\infty} \frac{d \eta}{E(\eta)^{p}} .
$$

Let us recall the following inequality [48, Theorem 4.1]

$$
\|f(x) g(-i \nabla)\|_{\mathfrak{S}_{p}} \leq(2 \pi)^{-3 / p}\|f\|_{L^{p}\left(\mathbb{R}^{3}\right)}\|g\|_{L^{p}\left(\mathbb{R}^{3}\right)},
$$

which implies

$$
\left\|\frac{1}{\left|D_{0}\right|^{a}} f\right\|_{\mathfrak{S}_{p}} \leq \frac{S_{p, a p}}{4 \pi}\|f\|_{L^{p}\left(\mathbb{R}^{3}\right)}
$$


On the other hand, we shall often use the following trick

$$
\begin{aligned}
\left(E(p)^{2}+\eta^{2}\right)\left(E(q)^{2}+\eta^{2}\right) & =(E(p) E(q))^{2}+\left(E(p)^{2}+E(q)^{2}\right) \eta^{2}+\eta^{4} \\
& \geq \frac{1}{4} E(p+q)^{2}+\frac{1}{2} E(p+q)^{2} \eta^{2} \\
& \geq \frac{1}{4} E(p+q)^{2} E(\eta)^{2}
\end{aligned}
$$

by Lemma 6. This implies

$$
\frac{1}{\sqrt{E(p)^{2}+\eta^{2}} \sqrt{E(q)^{2}+\eta^{2}}} \leq \frac{2}{E(p+q) E(\eta)} .
$$

Recall now that we have $Q_{2}=Q_{2,0}+Q_{1,1}+Q_{0,2}$ with

$$
\begin{aligned}
Q_{2,0}= & -\frac{1}{2 \pi} \int_{-\infty}^{+\infty} d \eta \frac{1}{D^{0}+i \eta} R \frac{1}{D^{0}+i \eta} R \frac{1}{D^{0}+i \eta} \\
Q_{1,1}= & \frac{1}{2 \pi} \int_{-\infty}^{+\infty} d \eta \frac{1}{D^{0}+i \eta} R \frac{1}{D^{0}+i \eta} \varphi^{\prime} \frac{1}{D^{0}+i \eta} \\
& \quad+\frac{1}{2 \pi} \int_{-\infty}^{+\infty} d \eta \frac{1}{D^{0}+i \eta} \varphi^{\prime} \frac{1}{D^{0}+i \eta} R \frac{1}{D^{0}+i \eta} \\
Q_{0,2}= & -\frac{1}{2 \pi} \int_{-\infty}^{+\infty} d \eta \frac{1}{D^{0}+i \eta} \varphi^{\prime} \frac{1}{D^{0}+i \eta} \varphi^{\prime} \frac{1}{D^{0}+i \eta} .
\end{aligned}
$$

We shall now treat each term separately.

Lemma 13. We have $\rho_{0,2}=0$ and the following estimates:

$$
\begin{gathered}
\left\|Q_{2,0}\right\|_{\mathcal{Q}} \leq 2^{5 / 2} K_{3 / 2}\left(C_{R}\right)^{2}\|Q\|_{\mathcal{Q}}^{2}, \quad\left\|Q_{1,1}\right\|_{\mathcal{Q}} \leq 4 S_{6} C_{6} K_{3 / 2} C_{R}\|Q\|_{\mathcal{Q}}\left\|\rho^{\prime}\right\|_{\mathfrak{C}} \\
\left\|Q_{0,2}\right\|_{\mathcal{Q}} \leq 2 \sqrt{10} S_{6,5} C_{M} C_{6}\left\|\rho^{\prime}\right\|_{\mathfrak{C}}^{2} \\
\left\|\rho_{2,0}\right\|_{\mathfrak{C}} \leq \frac{S_{6} C_{6}\left(C_{R}\right)^{2}}{\pi}\|Q\|_{\mathcal{Q}}^{2}, \quad\left\|\rho_{1,1}\right\|_{\mathfrak{C}} \leq 4 \frac{S_{6,5} C_{M} C_{6} C_{R}}{\pi}\left\|\rho^{\prime}\right\|_{\mathfrak{C}}\|Q\|_{\mathcal{Q}}
\end{gathered}
$$

and so

$$
\left\|\left(Q_{2}, \rho_{2}\right)\right\| \leq \kappa_{2}\left\|\left(Q, \rho^{\prime}\right)\right\|^{2}
$$

with

$$
\begin{gathered}
\kappa_{2}=C_{Q_{2}} C_{R} \sqrt{2}+2 \sqrt{\pi} C_{\rho_{2}}, \\
C_{Q_{2}}=\max \left(2^{3 / 2} K_{3 / 2}, \frac{S_{6} C_{6} K_{3 / 2}}{\sqrt{2 \pi}}, \frac{\sqrt{5} S_{6,5} C_{M} C_{6}}{\pi \sqrt{2}}\right) \\
C_{\rho_{2}}=\max \left(\frac{S_{6} C_{6}}{2 \pi}, \frac{S_{6,5} C_{M} C_{6}}{\pi^{3 / 2} \sqrt{2}}\right),
\end{gathered}
$$

where $C_{M}$ is a constant defined in Lemma 14 . 
Proof. Step 1 : Estimates on the exchange term $Q_{2}$.

- $Q_{2,0}$. To estimate $Q_{2,0}$, we write

$$
\left|\widehat{Q_{2,0}}(p, q)\right| \leq \frac{1}{2 \pi} \int_{-\infty}^{+\infty} d \eta \int_{\mathbb{R}^{3}} d p_{1} \frac{\left|\widehat{R}\left(p, p_{1}\right)\right|}{\sqrt{E(p)^{2}+\eta^{2}}} \frac{\left|\widehat{R}\left(p_{1}, q\right)\right|}{\sqrt{E\left(p_{1}\right)^{2}+\eta^{2}}} \frac{1}{\sqrt{E(q)^{2}+\eta^{2}}}
$$

and so by (58)

$$
\left|\widehat{Q_{2,0}}(p, q)\right| \leq \frac{2^{3 / 2}}{2 \pi} \int_{-\infty}^{+\infty} \frac{d \eta}{E(\eta)^{3 / 2} E(p+q)^{1 / 2}} \int_{\mathbb{R}^{3}} \frac{\left|\widehat{R}\left(p, p_{1}\right)\right|}{E\left(p+p_{1}\right)^{1 / 2}} \frac{\left|\widehat{R}\left(p_{1}, q\right)\right|}{E\left(p_{1}+q\right)^{1 / 2}} d p_{1}
$$

which implies

$$
\begin{aligned}
E(p-q) E(p+q)^{1 / 2}\left|\widehat{Q_{2,0}}(p, q)\right| \leq 2^{5 / 2} K_{3 / 2} \int_{\mathbb{R}^{3}} \frac{E\left(p-p_{1}\right)\left|\widehat{R}\left(p, p_{1}\right)\right|}{E\left(p+p_{1}\right)^{1 / 2}} \times \\
\times \frac{E\left(p_{1}-q\right)\left|\widehat{R}\left(p_{1}, q\right)\right|}{E\left(p_{1}+q\right)^{1 / 2}} d p_{1}
\end{aligned}
$$

and finally

$$
\left\|Q_{2,0}\right\|_{\mathcal{Q}} \leq 2^{5 / 2} K_{3 / 2}\|R\|_{\mathcal{R}}^{2} \leq 2^{5 / 2} K_{3 / 2}\left(C_{R}\right)^{2}\|Q\|_{\mathcal{Q}}^{2}
$$

- $Q_{1,1}$. We treat for instance

$$
Q_{1,1}^{\prime}:=\frac{1}{(2 \pi)^{5 / 2}} \int_{-\infty}^{+\infty} d \eta \frac{1}{D^{0}+i \eta} R \frac{1}{D^{0}+i \eta} \varphi^{\prime} \frac{1}{D^{0}+i \eta}
$$

and use the same method to obtain

$$
\begin{aligned}
& E(p-q) E(p+q)^{1 / 2}\left|\widehat{Q_{1,1}^{\prime}}(p, q)\right| \leq \frac{4}{(2 \pi)^{5 / 2}} \int_{-\infty}^{+\infty} \frac{d \eta}{E(\eta)} \times \\
\times & \int_{\mathbb{R}^{3}} \frac{E\left(p-p_{1}\right)}{E\left(p+p_{1}\right)^{1 / 2}}\left|\widehat{R}\left(p, p_{1}\right)\right| \frac{E\left(p_{1}-q\right)}{\left(E\left(p_{1}\right)^{2}+\eta^{2}\right)^{1 / 4}\left(E(q)^{2}+\eta^{2}\right)^{1 / 4}}\left|\widehat{\varphi^{\prime}}\left(p_{1}-q\right)\right| d p_{1} .
\end{aligned}
$$

This means that

$$
\left\|Q_{1,1}^{\prime}\right\|_{\mathcal{Q}} \leq \frac{4}{2 \pi} \int_{-\infty}^{+\infty} \frac{d \eta}{E(\eta)}\left\|R^{\prime} \frac{1}{\left(\left|D_{0}\right|^{2}+\eta^{2}\right)^{1 / 4}} f \frac{1}{\left(\left|D_{0}\right|^{2}+\eta^{2}\right)^{1 / 4}}\right\|_{\mathfrak{S}_{2}}
$$

where we have introduced $R^{\prime}$ and $f$ defined by

$$
\widehat{R^{\prime}}(p, q):=\frac{E(p-q)}{E(p+q)^{1 / 2}}|\widehat{R}(p, q)|, \quad \widehat{f}(k):=E(k)\left|\widehat{\varphi^{\prime}}(k)\right| .
$$

But now

$$
\begin{aligned}
\left\|R^{\prime} \frac{1}{\left(\left|D_{0}\right|^{2}+\eta^{2}\right)^{\frac{1}{4}}} f \frac{1}{\left(\left|D_{0}\right|^{2}+\eta^{2}\right)^{\frac{1}{4}}}\right\|_{\mathfrak{S}_{2}} & \leq\left\|R^{\prime}\right\|_{\mathfrak{S}_{2}}\left\|\frac{1}{\left(\left|D_{0}\right|^{2}+\eta^{2}\right)^{\frac{1}{4}}} f \frac{1}{\left(\left|D_{0}\right|^{2}+\eta^{2}\right)^{\frac{1}{4}}}\right\|_{\mathfrak{S}_{\infty}} \\
& \leq\|R\|_{\mathcal{R}}\left\|\frac{1}{\left(\left|D_{0}\right|^{2}+\eta^{2}\right)^{\frac{1}{4}}} f \frac{1}{\left(\left|D_{0}\right|^{2}+\eta^{2}\right)^{\frac{1}{4}}}\right\|_{\mathfrak{S}_{6}} .
\end{aligned}
$$


If we now use inequality (57), we obtain

$$
\begin{aligned}
\left\|\frac{1}{\left(\left|D_{0}\right|^{2}+\eta^{2}\right)^{\frac{1}{4}}} f \frac{1}{\left(\left|D_{0}\right|^{2}+\eta^{2}\right)^{\frac{1}{4}}}\right\|_{\mathfrak{S}_{6}} & \leq\left\|\frac{1}{\left(\left|D_{0}\right|^{2}+\eta^{2}\right)^{\frac{1}{4}}}|f|^{1 / 2}\right\|_{\mathfrak{S}_{12}}^{2} \\
& \leq(2 \pi)^{-1 / 2}\left(\int_{\mathbb{R}^{3}} \frac{d u}{\left.1+|u|^{2}+\eta^{2}\right)^{3}}\right)^{1 / 6}\|f\|_{L^{6}} \\
& =\frac{S_{6}}{4 \pi E(\eta)^{1 / 2}}\|f\|_{L^{6}} .
\end{aligned}
$$

Finally since

$$
\|f\|_{L^{6}} \leq C_{6}\|\nabla f\|_{L^{2}}=C_{6}\left\|\varphi^{\prime}\right\|_{\mathcal{Y}}
$$

and $\left\|\varphi^{\prime}\right\|_{\mathcal{Y}}=(4 \pi)\left\|\rho^{\prime}\right\|_{\mathfrak{C}}$, we obtain

$$
\left\|Q_{1,1}^{\prime}\right\|_{\mathcal{Q}} \leq \frac{4 S_{6} C_{6}}{2 \pi} \int_{-\infty}^{+\infty} \frac{d \eta}{E(\eta)^{3 / 2}}\|R\|_{\mathcal{R}}\left\|\rho^{\prime}\right\|_{\mathfrak{C}}
$$

and

$$
\left\|Q_{1,1}\right\|_{\mathcal{Q}} \leq 8 S_{6} C_{6} K_{3 / 2} C_{R}\|Q\|_{\mathcal{Q}}\left\|\rho^{\prime}\right\|_{\mathfrak{C}}
$$

- $Q_{0,2}$. Unfortunately, the method used above cannot be applied to $Q_{0,2}$. In this case, we have to calculate this term explicitely. We can write

$$
Q_{0,2}=\sum_{\epsilon_{1}, \epsilon_{2}, \epsilon_{3} \in\{ \pm\}} Q_{0,2}^{\epsilon_{1} \epsilon_{2} \epsilon_{3}}
$$

where for instance (by a residuum formula)

$$
\begin{gathered}
\widehat{Q_{0,2}^{+++}}(p, q)=\widehat{Q_{0,2}^{--}}(p, q)=0 \\
\widehat{Q_{0,2}^{+--}}(p, q)=(2 \pi)^{-3} \int_{\mathbb{R}^{3}} d p_{1} \frac{\Lambda^{+}(p) \widehat{\varphi^{\prime}}\left(p-p_{1}\right) \Lambda^{-}\left(p_{1}\right) \widehat{\varphi^{\prime}}\left(p_{1}-q\right) \Lambda^{-}(q)}{(E(p)+E(q))\left(E(p)+E\left(p_{1}\right)\right)} \\
\widehat{Q_{0,2}^{+-+}}(p, q)=(2 \pi)^{-3} \int_{\mathbb{R}^{3}} d p_{1} \frac{\Lambda^{+}(p) \widehat{\varphi^{\prime}}\left(p-p_{1}\right) \Lambda^{-}\left(p_{1}\right) \widehat{\varphi^{\prime}}\left(p_{1}-q\right) \Lambda^{+}(q)}{\left(E(p)+E\left(p_{1}\right)\right)\left(E(q)+E\left(p_{1}\right)\right)}
\end{gathered}
$$

and similar formulas for the other $Q_{0,2}^{\epsilon_{1} \epsilon_{2} \epsilon_{3}}$. We now treat for instance $\widehat{Q_{0,2}^{+-}}$. Using (30), we may obtain

$$
\begin{aligned}
& E(p-q) E(p+q)^{1 / 2}\left|\widehat{Q_{0,2}^{+-}}\right| \leq 2(2 \pi)^{-3} \int_{\mathbb{R}^{3}} d p_{1} \frac{E\left(p-p_{1}\right)}{E\left(p+p_{1}\right)^{2 / 3}} \times \\
& \times\left|\Lambda^{+}(p) \widehat{\varphi^{\prime}}\left(p-p_{1}\right) \Lambda^{-}\left(p_{1}\right)\right| \times \frac{E\left(p_{1}-q\right)\left|\widehat{\varphi^{\prime}}\left(p_{1}-q\right)\right|}{E\left(p_{1}\right)^{1 / 3} E(q)^{1 / 2}} d p_{1} .
\end{aligned}
$$

So, we may write

$$
\left\|Q_{0,2}^{+--}\right\|_{\mathcal{Q}} \leq 2\left\|M_{f} \frac{1}{\left|D_{0}\right|^{1 / 3}} f \frac{1}{\left|D_{0}\right|^{1 / 2}}\right\|_{\mathfrak{S}_{2}} \leq 2\left\|M_{f}\right\|_{\mathfrak{S}_{2}}\left\|\frac{1}{\left|D_{0}\right|^{1 / 3}} f \frac{1}{\left|D_{0}\right|^{1 / 2}}\right\|_{\mathfrak{S}_{\infty}},
$$

where

$$
\widehat{M_{f}}(p, q):=(2 \pi)^{-3 / 2} \frac{\widehat{f}(p-q)\left|\Lambda^{+}(p) \Lambda^{-}(q)\right|}{E(p+q)^{2 / 3}} .
$$


Lemma 14. When $M_{f}$ is defined by formula (60), then

$$
\left\|M_{f}\right\|_{\mathfrak{S}_{2}} \leq \frac{C_{M}}{4 \pi}\left(\int_{\mathbb{R}^{3}}|k|^{2}|\widehat{f}(k)|^{2} d k\right)^{1 / 2}
$$

where $C_{M}:=2\left(\int_{0}^{\infty} \frac{t^{2} d t}{E(2 t)^{4 / 3} E(t)^{2}}\right)^{1 / 2} \simeq 2.1589$.

Proof. We have $\left|\Lambda^{+}(p) \Lambda^{-}(q)\right|^{2}=\operatorname{Tr}_{\mathbb{C}^{4}}\left(\Lambda^{+}(p) \Lambda^{-}(q)\right)$ and so, by (54),

$$
\begin{aligned}
\iint d p d q\left|\widehat{M_{f}}(p, q)\right|^{2} & \leq(2 \pi)^{-3} \iint d p d q \frac{|p-q|^{2}|\widehat{f}(p-q)|^{2}}{2 E((p+q) / 2)^{2} E(p+q)^{4 / 3}} \\
& \leq(2 \pi)^{-3} \int d k|k|^{2}|\widehat{f}(k)|^{2} \int \frac{d u}{2 E(2 u)^{4 / 3} E(u)^{2}} \\
& \leq(2 \pi)^{-2}\left(\int d k|k|^{2}|\widehat{f}(k)|^{2}\right) \int_{0}^{\infty} \frac{t^{2} d t}{E(2 t)^{4 / 3} E(t)^{2}}
\end{aligned}
$$

Finally, since by (59)

$$
\left\|\frac{1}{\left|D_{0}\right|^{1 / 3}} f \frac{1}{\left|D_{0}\right|^{1 / 2}}\right\|_{\mathfrak{S}_{\infty}} \leq\left\|\frac{1}{\left|D_{0}\right|^{1 / 3}} f \frac{1}{\left|D_{0}\right|^{1 / 2}}\right\|_{\mathfrak{S}_{6}} \leq \frac{S_{6,5}}{4 \pi}\|f\|_{L^{6}},
$$

we obtain

$$
\left\|Q_{0,2}^{+--}\right\|_{\mathcal{Q}} \leq 2 S_{6,5} C_{M} C_{6}\left\|\rho^{\prime}\right\|_{\mathfrak{C}}^{2} .
$$

This result is immediately extended to the others terms and since we can prove

$$
|Q|^{2}=\left|Q^{++-}+Q^{+--}\right|^{2}+\left|Q^{-++}+Q^{--+}\right|^{2}+\left|Q^{+-+}\right|^{2}+\left|Q^{-+-}\right|^{2},
$$

we arrive at

$$
\left\|Q_{0,2}\right\|_{\mathcal{Q}} \leq 2 \sqrt{10} S_{6,5} C_{M} C_{6}\left\|\rho^{\prime}\right\|_{\mathfrak{C}^{*}}^{2}
$$

Step 2 : Estimates on the density $\rho_{2}$. Let us now treat the density $\rho_{2}$. The general idea of the proof is to estimate $\left\langle\rho_{2}, \zeta\right\rangle$ in terms of the norm $\|\zeta\|_{\mathfrak{C}^{\prime}}$ by using

$$
|\langle\rho, \zeta\rangle|=|\operatorname{Tr}(Q \zeta)|=|\operatorname{Tr}(\widehat{Q \zeta})|=\left|\int_{\mathbb{R}^{3}} \operatorname{Tr}_{\mathbb{C}^{4}}(\widehat{Q \zeta})(p, p) d p\right| \leq \int_{\mathbb{R}^{3}}|\widehat{Q \zeta}(p, p)| d p
$$

This can be done if we know that $Q \zeta \in \mathfrak{S}_{1}$. But we have

$$
\begin{array}{r}
\|Q \zeta\|_{\mathfrak{S}_{1}}=\left\|Q\left|D_{0}\right|^{2} \frac{1}{\left|D_{0}\right|^{2}} \zeta\right\|_{\mathfrak{S}_{1}} \leq\left\|Q\left|D_{0}\right|^{2}\right\|_{\mathfrak{S}_{2}}\left\|\frac{1}{\left|D_{0}\right|^{2}} \zeta\right\|_{\mathfrak{S}_{2}} \\
\leq E(\Lambda)^{2}\|Q\|_{\mathfrak{S}_{2}} \frac{S_{2,4}}{4 \pi}\|\zeta\|_{L^{2}}
\end{array}
$$

showing that $Q \zeta \in \mathfrak{S}_{1}$ when $\zeta \in L^{2}$. So, in what follows, we shall assume that $\zeta \in \mathfrak{C}^{\prime} \cap L^{2}$ and prove a bound depending only on $\|\zeta\|_{\mathfrak{C}^{\prime}}$. By the density of $\mathfrak{C}^{\prime} \cap L^{2}$ in $\mathfrak{C}^{\prime}$, this will give us a bound on $\|\rho\|_{\mathfrak{C}}$. 
Let us remark first that $\rho_{0,2}$ vanishes. Indeed we have

$$
\widehat{\rho_{0,2}}(k)=\frac{1}{(2 \pi)^{3 / 2}} \int_{|p| \leq \Lambda} \operatorname{Tr}_{\mathbb{C}^{4}}\left(\widehat{Q_{0,2}}(p+k / 2, p-k / 2)\right) d p
$$

and

$$
\begin{aligned}
& \operatorname{Tr}_{\mathbb{C}^{4}}\left(\widehat{Q_{0,2}}(p, q)\right)= \frac{1}{(2 \pi)^{4}} \int_{-\infty}^{+\infty} d \eta \int_{\mathbb{R}^{3}} d p_{1} \operatorname{Tr}_{\mathbb{C}^{4}}\left(\frac{1}{D_{0}(p)+i \eta} \widehat{\varphi^{\prime}}\left(p-p_{1}\right) \times\right. \\
&\left.\times \frac{1}{D_{0}\left(p_{1}\right)+i \eta} \widehat{\varphi^{\prime}}\left(p_{1}-q\right) \frac{1}{D_{0}(q)+i \eta}\right) \\
&=\frac{1}{(2 \pi)^{4}} \int_{-\infty}^{+\infty} d \eta \int_{\mathbb{R}^{3}} d p_{1} \frac{\widehat{\varphi^{\prime}}\left(p-p_{1}\right) \widehat{\varphi^{\prime}}\left(p_{1}-q\right)}{\sqrt{E(p)^{2}+\eta^{2}} \sqrt{E\left(p_{1}\right)^{2}+\eta^{2}} \sqrt{E(q)^{2}+\eta^{2}}} \times \\
&\left.\times \operatorname{Tr}_{\mathbb{C}^{4}}\left[\left(D_{0}(p)-i \eta\right)\right)\left(D_{0}\left(p_{1}\right)-i \eta\right)\left(D_{0}(q)-i \eta\right)\right] .
\end{aligned}
$$

Now the terms linear in the Dirac matrices are traceless and the remaining terms are odd in $\eta$ and vanish after integration. This can be easily generalized to $\rho_{0,2 k}$ for all $k$, and is known as Furry's Theorem in the physics literature 22 .

- $\rho_{2,0}$. We use here a method similar to what we have done above. We estimate for some $\zeta \in \mathfrak{C}^{\prime \prime} \cap L^{2}$ and $Q_{\zeta}:=Q_{2,0} \zeta$

$$
\begin{aligned}
\left|\widehat{Q_{\zeta}}(p, p)\right| \leq & (2 \pi)^{-5 / 2} \int_{-\infty}^{+\infty} d \eta \iint \frac{\left|\widehat{R}\left(p, p_{1}\right)\right|\left|\widehat{R}\left(p_{1}, p_{2}\right)\right| \widehat{\zeta}\left(p_{2}-p\right) \mid d p_{1} d p_{2}}{\sqrt{E(p)^{2}+\eta^{2}} \sqrt{E\left(p_{1}\right)^{2}+\eta^{2}} \sqrt{E\left(p_{2}\right)^{2}+\eta^{2}}}, \\
\leq & 4(2 \pi)^{-5 / 2} \int_{-\infty}^{+\infty} \frac{d \eta}{E(\eta)} \iint d p_{1} d p_{2} \frac{E\left(p-p_{1}\right)\left|\widehat{R}\left(p, p_{1}\right)\right| E\left(p_{1}-p_{2}\right)\left|\widehat{R}\left(p_{1}, p_{2}\right)\right|}{E\left(p+p_{1}\right)^{1 / 2} E\left(p_{1}+p_{2}\right)^{1 / 2}} \times \\
& \times \frac{\left|\widehat{\zeta}\left(p_{2}-p\right)\right|}{E\left(p_{2}-p\right)\left(E(p)^{2}+\eta^{2}\right)^{1 / 4}\left(E\left(p_{2}\right)^{2}+\eta^{2}\right)^{1 / 4}}, \\
\leq & 4(2 \pi)^{-5 / 2} \int_{-\infty}^{+\infty} \frac{d \eta}{E(\eta)} \iint d p_{1} d p_{2} \frac{R^{\prime}\left(p, p_{1}\right) R^{\prime}\left(p_{1}, p_{2}\right) \widehat{\zeta^{\prime}}\left(p_{2}-p\right)}{\left(E(p)^{2}+\eta^{2}\right)^{1 / 4}\left(E\left(p_{2}\right)^{2}+\eta^{2}\right)^{1 / 4}}
\end{aligned}
$$

where $\widehat{R^{\prime}}(p, q)=\frac{E(p-q)|\widehat{R}(p, q)|}{E(p+q)^{1 / 2}}$ and $\widehat{\zeta^{\prime}}(k)=E(k)^{-1} \widehat{\zeta}(k)$. This means that

$$
\begin{aligned}
\left|\left\langle\rho_{2,0}, \zeta\right\rangle\right| & \leq \frac{4}{2 \pi} \int_{-\infty}^{+\infty} \frac{d \eta}{E(\eta)}\left\|R^{\prime} R^{\prime} \frac{1}{\left(\left|D_{0}\right|^{2}+\eta^{2}\right)^{1 / 4}} \zeta^{\prime} \frac{1}{\left(\left|D_{0}\right|^{2}+\eta^{2}\right)^{1 / 4}}\right\|_{\mathfrak{S}_{1}} \\
& \leq \frac{4 S_{6}}{(2 \pi)(4 \pi)} \int_{-\infty}^{+\infty} \frac{d \eta}{E(\eta)^{3 / 2}}\left\|R^{\prime}\right\|_{\mathfrak{S}_{2}}^{2}\left\|\zeta^{\prime}\right\|_{L^{6}} \leq \frac{S_{6} C_{6}\left(C_{R}\right)^{2} K_{3 / 2}}{\pi}\|Q\|_{\mathcal{Q}}^{2}\|\zeta\|_{\mathfrak{C}^{\prime}}
\end{aligned}
$$

by (59), showing that

$$
\left\|\rho_{2,0}\right\|_{\mathfrak{C}} \leq \frac{S_{6} C_{6}\left(C_{R}\right)^{2}}{\pi} K_{3 / 2}\|Q\|_{\mathcal{Q}}^{2}
$$


- $\rho_{1,1}$. Unfortunately, as for $Q_{0,2}$, we have to calculate $\rho_{1,1}$ explicitely. Let us start for instance with $\rho_{1,1}^{+--}$, the density associated with one of the two terms of $Q_{1,1}$

$$
(2 \pi)^{-3 / 2} \int_{\mathbb{R}^{3}} d p_{1} \frac{\Lambda^{+}(p) \widehat{R}\left(p-p_{1}\right) \Lambda^{-}\left(p_{1}\right) \widehat{\varphi^{\prime}}\left(p_{1}-q\right) \Lambda^{-}(q)}{(E(p)+E(q))\left(E(p)+E\left(p_{1}\right)\right)} .
$$

We use the same method as above and estimate for some $\zeta \in \mathfrak{C}^{\prime} \cap L^{2}$ the term $\widehat{Q_{\zeta}}(p, p)=(2 \pi)^{-3} \iint d p_{1} d p_{2} \frac{\Lambda^{+}(p) \widehat{R}\left(p, p_{1}\right) \Lambda^{-}\left(p_{1}\right) \widehat{\varphi^{\prime}}\left(p_{1}-p_{2}\right) \Lambda^{-}\left(p_{2}\right)}{\left(E(p)+E\left(p_{2}\right)\right)\left(E(p)+E\left(p_{1}\right)\right)} \widehat{\zeta}\left(p_{2}-p\right)$, by

$$
\begin{aligned}
\left|\widehat{Q_{\zeta}}(p, p)\right| \leq(2 \pi)^{-3} \iint \frac{\left|\Lambda^{+}(p) \widehat{R}\left(p, p_{1}\right) \Lambda^{-}\left(p_{1}\right)\right|}{E\left(p+p_{1}\right)^{1 / 2}} \frac{\left|\widehat{\varphi}^{\prime}\left(p_{1}-p_{2}\right)\right|}{E\left(p_{1}\right)^{1 / 2} E\left(p_{2}\right)^{1 / 3}} \times \\
\times \frac{\left|\widehat{\zeta}\left(p_{2}-p\right)\right| \times\left|\Lambda^{-}\left(p_{2}\right) \Lambda^{+}(p)\right|}{E\left(p+p_{2}\right)^{2 / 3}} d p_{1} d p_{2}, \\
\leq 2(2 \pi)^{-3 / 2} \iint \frac{E\left(p-p_{1}\right)\left|\Lambda^{+}(p) \widehat{R}\left(p, p_{1}\right) \Lambda^{-}\left(p_{1}\right)\right|}{E\left(p+p_{1}\right)^{1 / 2}} \times \\
\times \frac{\widehat{f}\left(p_{1}-p_{2}\right)}{E\left(p_{1}\right)^{1 / 2} E\left(p_{2}\right)^{1 / 3}} \widehat{M_{\zeta^{\prime}}}\left(p_{2}, p\right) d p_{1} d p_{2},
\end{aligned}
$$

with $\widehat{f}(k):=E(k)\left|\widehat{\varphi^{\prime}}(k)\right|$ and $\widehat{\zeta^{\prime}}(k):=|\widehat{\zeta}(k)| / E(k)$. Now

$$
\left|\widehat{Q_{\zeta}}(p, p)\right| \leq 2(2 \pi)^{-3 / 2} \iint \widehat{R_{1}}\left(p, p_{1}\right) \frac{\widehat{f}\left(p_{1}-p_{2}\right)}{E\left(p_{1}\right)^{1 / 2} E\left(p_{2}\right)^{1 / 3}} \widehat{M_{\zeta^{\prime}}}\left(p_{2}, p\right) d p_{1} d p_{2}
$$

with

$$
\widehat{R_{1}}\left(p, p_{1}\right):=\frac{E\left(p-p_{1}\right)\left|\Lambda^{+}(p) \widehat{R}\left(p, p_{1}\right) \Lambda^{-}\left(p_{1}\right)\right|}{E\left(p+p_{1}\right)^{1 / 2}} .
$$

We thus have

$$
\begin{aligned}
\left|\left\langle\rho_{1,1}^{+--}, \zeta\right\rangle\right| & \leq 2\left\|R_{1} \frac{1}{\left|D_{0}\right|^{1 / 2}} f \frac{1}{\left|D_{0}\right|^{1 / 3}} M_{\zeta^{\prime}}\right\|_{\mathfrak{S}^{1}} \\
& \leq 2\left\|\Lambda^{+} R \Lambda^{-}\right\|_{\mathcal{R}}\left\|M_{\zeta^{\prime}}\right\|_{\mathfrak{S}_{2}}\left\|\frac{1}{\left|D_{0}\right|^{1 / 2}} f \frac{1}{\left|D_{0}\right|^{1 / 3}}\right\|_{\mathfrak{S}_{\infty}} \\
& \leq 2\left\|\Lambda^{+} R \Lambda^{-}\right\|_{\mathcal{R}}\left\|M_{\zeta^{\prime}}\right\|_{\mathfrak{S}_{2}}\left\|\frac{1}{\left|D_{0}\right|^{1 / 2}} f \frac{1}{\left|D_{0}\right|^{1 / 3}}\right\|_{\mathfrak{S}_{6}} \\
& \leq \frac{S_{6,5} C_{M} C_{6}}{2 \pi}\left\|\Lambda^{+} R \Lambda^{-}\right\|_{\mathcal{R}}\|\zeta\|_{\mathfrak{C}^{\prime}}\left\|\rho^{\prime}\right\|_{\mathfrak{C}}
\end{aligned}
$$

and finally

$$
\left\|\rho_{1,1}^{+--}\right\|_{\mathfrak{C}} \leq \frac{S_{6,5} C_{M} C_{6}}{2 \pi}\left\|\Lambda^{+} R \Lambda^{-}\right\|_{\mathcal{R}}\left\|\rho^{\prime}\right\|_{\mathfrak{C}}
$$

We now treat $\rho_{1,1}^{+-+}$and estimate

$$
\widehat{Q_{\zeta}}(p, p)=(2 \pi)^{-3} \iint d p_{1} d p_{2} \frac{\Lambda^{+}(p) \widehat{R}\left(p, p_{1}\right) \Lambda^{-}\left(p_{1}\right) \widehat{\varphi^{\prime}}\left(p_{1}-p_{2}\right) \Lambda^{+}\left(p_{2}\right)}{\left(E(p)+E\left(p_{1}\right)\right)\left(E\left(p_{1}\right)+E\left(p_{2}\right)\right)} \widehat{\zeta}\left(p_{2}-p\right),
$$


by

$$
\begin{aligned}
\left|\widehat{Q_{\zeta}}(p, p)\right| \leq 2(2 \pi)^{-3} \iint \frac{E\left(p-p_{1}\right)\left|\Lambda^{+}(p) \widehat{R}\left(p, p_{1}\right) \Lambda^{-}\left(p_{1}\right)\right|}{E\left(p+p_{1}\right)^{1 / 2}} \times \\
\times \frac{\widehat{f}\left(p_{1}-p_{2}\right)\left|\Lambda^{-}\left(p_{1}\right) \Lambda^{+}\left(p_{2}\right)\right|}{E\left(p_{1}+p_{2}\right)^{2 / 3}} \frac{\widehat{\zeta^{\prime}}\left(p_{2}-p\right)}{E\left(p_{2}\right)^{5 / 6}} d p_{1} d p_{2} .
\end{aligned}
$$

Using the same argument as above, we arrive at

$$
\left\|\rho_{1,1}^{+-+}\right\|_{\mathfrak{C}} \leq \frac{S_{6,5} C_{M} C_{6}}{2 \pi}\left\|\Lambda^{+} R \Lambda^{-}\right\|_{\mathcal{R}}\left\|\rho^{\prime}\right\|_{\mathfrak{C}}
$$

To treat $\rho_{1,1}^{++-}$, we remark that

$$
\begin{aligned}
\frac{1}{\left(E(p)+E\left(p_{2}\right)\right)\left(E\left(p_{1}\right)+E\left(p_{2}\right)\right)} \leq \frac{1}{(E(p)} & \left.+E\left(p_{1}\right)\right)\left(E\left(p_{1}\right)+E\left(p_{2}\right)\right) \\
& +\frac{1}{\left(E(p)+E\left(p_{1}\right)\right)\left(E(p)+E\left(p_{2}\right)\right)}
\end{aligned}
$$

and use the same estimates as above to get

$$
\left\|\rho_{1,1}^{++-}\right\|_{\mathfrak{C}} \leq \frac{S_{6,5} C_{M} C_{6}}{\pi}\left\|\Lambda^{+} R \Lambda^{-}\right\|_{\mathcal{R}}\left\|\rho^{\prime}\right\|_{\mathfrak{C}}
$$

Finally, since $\sum_{\epsilon_{1}, \epsilon_{2} \in\{ \pm\}}\left\|\Lambda^{\epsilon_{1}} R \Lambda^{\epsilon_{2}}\right\|_{\mathcal{R}}^{2}=\|R\|_{\mathcal{R}}^{2}$, we end up with

$$
\left\|\rho_{1,1}\right\|_{\mathfrak{C}} \leq 2 \frac{S_{6,5} C_{M} C_{6}}{\pi}\left\|\rho^{\prime}\right\|_{\mathfrak{C}}\left(\sum_{\epsilon_{1}, \epsilon_{2} \in\{ \pm\}}\left\|\Lambda^{\epsilon_{1}} R \Lambda^{\epsilon_{2}}\right\|_{\mathcal{R}}\right) \leq 4 \frac{S_{6,5} C_{M} C_{6} C_{R}}{\pi}\left\|\rho^{\prime}\right\|_{\mathfrak{C}}\left\|_{Q}\right\|_{\mathcal{Q}}
$$

\subsubsection{The general $n^{\text {th }}$ order case}

Now that we have explained how the proof works for the second order, let us estimate the general $n^{\text {th }}$ order term.

Lemma 15. We have the following estimates

$$
\begin{gathered}
\forall n \geq 3, \quad\left\|Q_{n}\right\|_{\mathcal{Q}} \leq n K_{\frac{n}{2}} C_{Q}\left\|\left(Q, \rho^{\prime}\right)\right\|^{n}, \quad \text { with } C_{Q}=\sqrt{2}\left(\frac{S_{6} C_{6}}{2 \sqrt{\pi}}\right)^{3}, \\
\forall n \geq 5, \quad\left\|\rho_{n}\right\|_{\mathfrak{C}} \leq n K_{\frac{n+1}{2}} C_{\rho}\left\|\left(Q, \rho^{\prime}\right)\right\|^{n}, \quad \text { with } C_{\rho}=\frac{S_{6} C_{6}}{4 \pi}\left(\frac{S_{6} C_{6}}{2 \sqrt{\pi}}\right)^{5}, \\
\left\|\rho_{4}\right\|_{\mathfrak{C}} \leq C_{\rho_{4}}\left\|\left(Q, \rho^{\prime}\right)\right\|^{4}, \quad \text { with } C_{\rho_{4}}:=\frac{K_{2} S_{6} C_{6}}{\pi}\left(\frac{S_{6} C_{6}}{2 \sqrt{\pi}}\right)^{2} .
\end{gathered}
$$

Therefore,

$$
\forall n \geq 4, \quad\left\|\left(Q_{n}, \rho_{n}\right)\right\| \leq \kappa_{n}\left\|\left(Q, \rho^{\prime}\right)\right\|^{n}
$$

with

$$
\kappa_{4}=4 C_{R} K_{2} C_{Q} \sqrt{2}+2 \sqrt{\pi} C_{\rho_{4}}, \quad \kappa_{n}=n C_{R} K_{\frac{n}{2}} C_{Q} \sqrt{2}+2 n K_{\frac{n+1}{2}} C_{\rho} \sqrt{\pi}
$$


Remark that it can be proved that $K_{n} \sim_{n \rightarrow \infty} \frac{C}{\sqrt{n}}$, which gives the claimed behaviour for $\kappa_{n}$ as $n \rightarrow \infty$.

Proof. Step 1 : Estimates on the exchange term $Q_{n}$.

- $Q_{k, l}$ with $k \geq 1$ and $k+l=n \geq 3$. Recall that

$$
Q_{k, l}=\frac{(-1)^{l+1}}{2 \pi} \sum_{I \cup J=\{1, \ldots, n\},|I|=k,|J|=l} \int_{-\infty}^{+\infty} d \eta \frac{1}{D^{0}+i \eta} \prod_{j=1}^{n}\left(R_{j} \frac{1}{D^{0}+i \eta}\right),
$$

where $R_{j}=R$ if $j \in I$ and $R_{j}=\varphi^{\prime}$ if $j \in J$. For the sake of simplicity, we treat only

$$
Q_{k, l}^{\prime}=\frac{1}{2 \pi} \int_{-\infty}^{+\infty} d \eta \frac{1}{D^{0}+i \eta}\left(R \frac{1}{D^{0}+i \eta}\right)^{k}\left(\varphi^{\prime} \frac{1}{D^{0}+i \eta}\right)^{l} .
$$

We have

$$
\begin{aligned}
&\left|\widehat{Q_{k, l}^{\prime}}(p, q)\right| \leq \frac{1}{(2 \pi)^{1+\frac{3 l}{2}}} \int_{-\infty}^{+\infty} d \eta \int \cdots \int \frac{1}{\left(E(p)^{2}+\eta^{2}\right)^{1 / 2}}\left|\widehat{R}\left(p, p_{1}\right)\right| \times \\
& \times \frac{1}{\left(E\left(p_{1}\right)^{2}+\eta^{2}\right)^{1 / 4}} \prod_{j=1}^{k-1}\left(\frac{1}{\left(E\left(p_{j}\right)^{2}+\eta^{2}\right)^{1 / 4}}\left|\widehat{R}\left(p_{j}, p_{j+1}\right)\right| \frac{1}{\left(E\left(p_{j+1}\right)^{2}+\eta^{2}\right)^{1 / 4}}\right) \times \\
& \times \prod_{j=k}^{n-2}\left(\frac{1}{\left(E\left(p_{j}\right)^{2}+\eta^{2}\right)^{1 / 4}}\left|\widehat{\varphi}^{\prime}\left(p_{j}-p_{j+1}\right)\right| \frac{1}{\left(E\left(p_{j+1}\right)^{2}+\eta^{2}\right)^{1 / 4}}\right) \times \\
& \quad \times \frac{1}{\left(E\left(p_{n-1}\right)^{2}+\eta^{2}\right)^{1 / 4}}\left|\widehat{\varphi}^{\prime}\left(p_{n-1}-q\right)\right| \frac{1}{\left(E(q)^{2}+\eta^{2}\right)^{1 / 2}} d p_{1} \cdots d p_{n-1}
\end{aligned}
$$

so by (58),

$$
\begin{gathered}
E(p-q) E(p+q)^{1 / 2}\left|\widehat{Q_{k, l}^{\prime}}(p, q)\right| \leq \frac{2^{\frac{k+1}{2}} E(p-q)}{(2 \pi)^{1+\frac{3 l}{2}}} \int_{-\infty}^{+\infty} \frac{d \eta}{E(\eta)^{\frac{k+1}{2}}} \int \cdots \int \frac{\left|\widehat{R}\left(p, p_{1}\right)\right|}{E\left(p+p_{1}\right)^{1 / 2}} \times \\
\times \prod_{j=1}^{k-1} \frac{\left|\widehat{R}\left(p_{j}, p_{j+1}\right)\right|}{E\left(p_{j}+p_{j+1}\right)^{1 / 2}} \prod_{j=k}^{n-2}\left(\frac{1}{\left(E\left(p_{j}\right)^{2}+\eta^{2}\right)^{1 / 4}}\left|\widehat{\varphi^{\prime}}\left(p_{j}-p_{j+1}\right)\right| \frac{1}{\left(E\left(p_{j+1}\right)^{2}+\eta^{2}\right)^{1 / 4}}\right) \times \\
\times \frac{1}{\left(E\left(p_{n-1}\right)^{2}+\eta^{2}\right)^{1 / 4}}\left|\widehat{\varphi^{\prime}}\left(p_{n-1}-q\right)\right| \frac{1}{\left(E(q)^{2}+\eta^{2}\right)^{1 / 4}} d p_{1} \cdots d p_{n-1} .
\end{gathered}
$$

Now if we use the easy generalization of (28),

$$
E(p-q) \leq E\left(p-p_{1}\right)+E\left(p_{1}-p_{2}\right)+\cdots+E\left(p_{n-2}-p_{n-1}\right)+E\left(p_{n-1}-q\right),
$$

we obtain by a similar argument as before

$$
\begin{aligned}
\left\|Q_{k, l}^{\prime}\right\|_{\mathcal{Q}} \leq \frac{2^{\frac{k+1}{2}}}{2 \pi}\left[k\left(C_{R}\right)^{k}\left(4 \pi C_{\infty}\right)^{l}\left(\int_{-\infty}^{\infty} \frac{d \eta}{E(\eta)^{l+\frac{k+1}{2}}}\right)\right. \\
\left.\quad+l\left(C_{R}\right)^{k}\left(4 \pi C_{\infty}\right)^{l-1} S_{6} C_{6}\left(\int_{-\infty}^{\infty} \frac{d \eta}{E(\eta)^{l+\frac{k}{2}}}\right)\right]\|Q\|_{\mathcal{Q}}^{k}\left\|\rho^{\prime}\right\|_{\mathfrak{C}}^{l} .
\end{aligned}
$$


To obtain this result, we have estimated each term containing a $\varphi^{\prime}$ by using

$$
\left\|\frac{1}{\left(\left|D_{0}\right|^{2}+E(\eta)^{2}\right)^{1 / 4}} \varphi^{\prime} \frac{1}{\left(\left|D_{0}\right|^{2}+E(\eta)^{2}\right)^{1 / 4}}\right\|_{\mathfrak{S}_{\infty}} \leq \frac{1}{E(\eta)}\left\|\varphi^{\prime}\right\|_{L^{\infty}} \leq \frac{C_{\infty}}{E(\eta)}\left\|\varphi^{\prime}\right\|_{\mathcal{Y}}
$$

and when $E\left(p_{j}-p_{j+1}\right)$ appears in front of a $\widehat{\varphi^{\prime}}\left(p_{j}-p_{j+1}\right)$ (i.e. when $j \geq k$ ), by using

$$
\begin{aligned}
& \left\|\frac{1}{\left(\left|D_{0}\right|^{2}+E(\eta)^{2}\right)^{1 / 4}} f \frac{1}{\left(\left|D_{0}\right|^{2}+E(\eta)^{2}\right)^{1 / 4}}\right\|_{\mathfrak{S}_{\infty}} \\
& \quad \leq\left\|\frac{1}{\left(\left|D_{0}\right|^{2}+E(\eta)^{2}\right)^{1 / 4}} f \frac{1}{\left(\left|D_{0}\right|^{2}+E(\eta)^{2}\right)^{1 / 4}}\right\|_{\mathfrak{S}_{6}} \leq \frac{S_{6}}{E(\eta)^{1 / 2} 4 \pi}\|f\|_{L^{6}} .
\end{aligned}
$$

So we have

$$
\begin{aligned}
\left\|Q_{k, l}^{\prime}\right\|_{\mathcal{Q}} & \leq 2^{\frac{k+1}{2}}\left[k\left(C_{R}\right)^{k}\left(4 \pi C_{\infty}\right)^{l} K_{l+\frac{k+1}{2}}+l\left(C_{R}\right)^{k}\left(4 \pi C_{\infty}\right)^{l-1} S_{6} C_{6} K_{l+\frac{k}{2}}\right]\|Q\|_{\mathcal{Q}}^{k}\left\|\rho^{\prime}\right\|_{\mathfrak{C}}^{l} \\
& \leq 2^{\frac{k+1}{2}} n\left(C_{R}\right)^{k}\left(4 \pi C_{\infty}\right)^{l} K_{l+\frac{k}{2}} \max \left(1, \frac{S_{6} C_{6}}{4 \pi C_{\infty}}\right)\|Q\|_{\mathcal{Q}}^{k}\left\|\rho^{\prime}\right\|_{\mathfrak{C}}^{l}
\end{aligned}
$$

which implies

$$
\left\|Q_{k, l}\right\|_{\mathcal{Q}} \leq\left(\begin{array}{l}
n \\
k
\end{array}\right) 2^{\frac{k+1}{2}} n\left(C_{R}\right)^{k}\left(4 \pi C_{\infty}\right)^{l} K_{l+\frac{k}{2}} \max \left(1, \frac{S_{6} C_{6}}{4 \pi C_{\infty}}\right)\|Q\|_{\mathcal{Q}}^{k}\left\|\rho^{\prime}\right\|_{\mathfrak{C}}^{l} .
$$

- $Q_{0, n}$ with $n \geq 3$. Recall that

$$
Q_{0, n}=\frac{(-1)^{n+1}}{2 \pi} \int_{-\infty}^{+\infty} d \eta \frac{1}{D^{0}+i \eta}\left(\varphi^{\prime} \frac{1}{D^{0}+i \eta}\right)^{n}
$$

so that

$$
\begin{gathered}
E(p-q) E(p+q)^{1 / 2}\left|\widehat{Q_{0, n}}(p, q)\right| \leq \frac{E(p-q)}{(2 \pi)^{1+\frac{3 n}{2}}} \int_{-\infty}^{+\infty} \frac{d \eta}{E(\eta)^{\frac{1}{2}}} \int \cdots \int d p_{1} \cdots d p_{n-1} \times \\
\times \frac{\left|\widehat{\varphi^{\prime}}\left(p-p_{1}\right)\right|}{\left(E(p)^{2}+\eta^{2}\right)^{1 / 4}\left(E\left(p_{1}\right)^{2}+\eta^{2}\right)^{1 / 4}} \times \\
\times \prod_{j=1}^{n-2}\left(\frac{1}{\left(E\left(p_{j}\right)^{2}+\eta^{2}\right)^{1 / 4}}\left|\widehat{\varphi}^{\prime}\left(p_{j}-p_{j+1}\right)\right| \frac{1}{\left(E\left(p_{j+1}\right)^{2}+\eta^{2}\right)^{1 / 4}}\right) \times \\
\times \frac{1}{\left(E\left(p_{n-1}\right)^{2}+\eta^{2}\right)^{1 / 4}}\left|\widehat{\varphi^{\prime}}\left(p_{n-1}-q\right)\right| \frac{1}{\left(E(q)^{2}+\eta^{2}\right)^{1 / 4}} .
\end{gathered}
$$

We now use (59) to bound for some $f_{1}, f_{2}$ and $f_{3}$

$$
\left\|\prod_{j=1}^{3}\left(\frac{1}{\left(\left|D_{0}\right|^{2}+\eta^{2}\right)^{1 / 4}} f_{j} \frac{1}{\left(\left|D_{0}\right|^{2}+\eta^{2}\right)^{1 / 4}}\right)\right\|_{\mathfrak{S}^{2}} \leq \frac{\left(S_{6}\right)^{3}}{E(\eta)^{3 / 2}(4 \pi)^{3}} \prod_{j=1}^{3}\left\|f_{j}\right\|_{L^{6}}
$$


to obtain

$$
\left\|Q_{0, n}\right\|_{\mathcal{Q}} \leq n \frac{\sqrt{2}}{2 \pi}\left(\int_{-\infty}^{\infty} \frac{d \eta}{E(\eta)^{2+(n-3)}}\right)\left(S_{6} C_{6}\right)^{3}\left(4 \pi C_{\infty}\right)^{n-3}\left\|\rho^{\prime}\right\|_{\mathfrak{C}^{\mathfrak{c}}}
$$

or

$$
\left\|Q_{0, n}\right\|_{\mathcal{Q}} \leq n K_{n-1} \sqrt{2}\left(S_{6} C_{6}\right)^{3}\left(4 \pi C_{\infty}\right)^{n-3}\left\|\rho^{\prime}\right\|_{\mathfrak{C}}^{n}
$$

Finally, we can write for instance (recall that $C_{\infty}=1 /(2 \sqrt{\pi})$ )

$$
\left\|Q_{n}\right\|_{\mathcal{Q}} \leq n K_{n / 2} C_{Q}\left(C_{R} \sqrt{2}\|Q\|_{\mathcal{Q}}+2 \sqrt{\pi}\left\|\rho^{\prime}\right\|_{\mathfrak{C}}\right)^{n}
$$

where

$$
C_{Q}=\sqrt{2} \max \left(1, \frac{S_{6} C_{6}}{4 \pi C_{\infty}},\left(\frac{S_{6} C_{6}}{4 \pi C_{\infty}}\right)^{3}\right)=\sqrt{2} \frac{\left(S_{6} C_{6}\right)^{3}}{8 \pi^{3 / 2}}
$$

and since $K_{n / 2} \geq K_{n-1}$ when $n \geq 2$.

Step 2 : Estimates on the density $\rho_{n}$.

- $\rho_{k, l}$ with $k \geq 2$ and $n \geq 3$. As before we treat for instance the density $\rho_{k, l}^{\prime}$ of the $Q_{k, l}^{\prime}$ where the $k R$ 's are on the left and the $l \varphi^{\prime}$ 's are on the right. For some fixed $\zeta \in \mathfrak{C}^{\prime} \cap L^{2}$, we introduce $Q_{\zeta}:=Q_{k, l}^{\prime} \zeta$. We thus estimate

$$
\begin{gathered}
\left|\widehat{Q_{\zeta}}(p, p)\right| \leq \frac{1}{(2 \pi)^{1+\frac{3(l+1)}{2}}} \int_{-\infty}^{+\infty} d \eta \int \cdots \int \frac{1}{\left(E(p)^{2}+\eta^{2}\right)^{1 / 4}}\left|\widehat{R}\left(p, p_{1}\right)\right| \times \\
\times \frac{1}{\left(E\left(p_{1}\right)^{2}+\eta^{2}\right)^{1 / 4}} \prod_{j=1}^{k-1}\left(\frac{1}{\left(E\left(p_{j}\right)^{2}+\eta^{2}\right)^{1 / 4}}\left|\widehat{R}\left(p_{j}, p_{j+1}\right)\right| \frac{1}{\left(E\left(p_{j+1}\right)^{2}+\eta^{2}\right)^{1 / 4}}\right) \times \\
\times \prod_{j=k}^{n-1}\left(\frac{1}{\left(E\left(p_{j}\right)^{2}+\eta^{2}\right)^{1 / 4}}\left|\widehat{\varphi^{\prime}}\left(p_{j}-p_{j+1}\right)\right| \frac{1}{\left(E\left(p_{j+1}\right)^{2}+\eta^{2}\right)^{1 / 4}}\right) \times \\
\quad \times \frac{1}{\left(E\left(p_{n}\right)^{2}+\eta^{2}\right)^{1 / 4}}\left|\widehat{\zeta}\left(p_{n}-p\right)\right| \frac{1}{\left(E(p)^{2}+\eta^{2}\right)^{1 / 4}} d p_{1} \cdots d p_{n} .
\end{gathered}
$$

We now use as before

$$
\left|\widehat{\zeta}\left(p_{n}-p\right)\right| \leq \frac{\left|\widehat{\zeta}\left(p_{n}-p\right)\right|}{E\left(p_{n}-p\right)}\left(E\left(p-p_{1}\right)+E\left(p_{1}-p_{2}\right)+\cdots+E\left(p_{n-1}-p_{n}\right)\right)
$$

to obtain

$$
\left|\left\langle\rho_{k, l}^{\prime}, \zeta\right\rangle\right| \leq n \frac{2^{k / 2} S_{6} C_{6}}{4 \pi}\left(C_{R}\right)^{k}\left(4 \pi C_{\infty}\right)^{l}\left(\frac{1}{2 \pi} \int_{-\infty}^{\infty} \frac{d \eta}{E(\eta)^{l+\frac{k+1}{2}}}\right)\|Q\|_{\mathcal{Q}}^{k}\left\|\rho^{\prime}\right\|_{\mathfrak{C}}^{l}\|\zeta\|_{\mathfrak{C}^{\prime}}
$$

and so

$$
\left\|\rho_{k, l}\right\|_{\mathfrak{C}} \leq n\left(\begin{array}{c}
n \\
k
\end{array}\right) \frac{S_{6} C_{6}}{4 \pi}\left(C_{R} \sqrt{2}\right)^{k}\left(4 \pi C_{\infty}\right)^{l} K_{l+\frac{k+1}{2}}\|Q\|_{\mathcal{Q}}^{k}\left\|\rho^{\prime}\right\|_{\mathfrak{C}}^{l}
$$


- $\rho_{1, l}$ with $l \geq 2$. We may treat for instance with the same notation as before

$$
\begin{aligned}
\left|\widehat{Q_{\zeta}}(p, p)\right| \leq \frac{1}{(2 \pi)^{1+\frac{3 n}{2}}} \int_{-\infty}^{+\infty} d \eta \int \cdots \int \frac{1}{\left(E(p)^{2}+\eta^{2}\right)^{1 / 4}}\left|\widehat{R}\left(p, p_{1}\right)\right| \times \\
\times \frac{1}{\left(E\left(p_{1}\right)^{2}+\eta^{2}\right)^{1 / 4}} \prod_{j=1}^{n-1}\left(\frac{1}{\left(E\left(p_{j}\right)^{2}+\eta^{2}\right)^{1 / 4}}\left|\widehat{\varphi^{\prime}}\left(p_{j}-p_{j+1}\right)\right| \frac{1}{\left(E\left(p_{j+1}\right)^{2}+\eta^{2}\right)^{1 / 4}}\right) \times \\
\times \frac{1}{\left(E\left(p_{n}\right)^{2}+\eta^{2}\right)^{1 / 4}}\left|\widehat{\zeta}\left(p_{n}-p\right)\right| \frac{1}{\left(E(p)^{2}+\eta^{2}\right)^{1 / 4}} d p_{1} \cdots d p_{n} .
\end{aligned}
$$

We now use (61) and obtain

$$
\left|\left\langle\rho_{1, l}^{\prime}, \zeta\right\rangle\right| \leq n \frac{2^{1 / 2}\left(S_{6} C_{6}\right)^{3}}{4 \pi} C_{R}\left(4 \pi C_{\infty}\right)^{l-2}\left(\frac{1}{2 \pi} \int_{-\infty}^{\infty} \frac{d \eta}{E(\eta)^{l-2+\frac{4}{2}}}\right)\|Q\|_{\mathcal{Q}}\left\|\rho^{\prime}\right\|_{\mathfrak{C}^{l}}^{l}\|\zeta\|_{\mathfrak{C}^{\prime}}
$$

and so

$$
\left\|\rho_{1, l}\right\|_{\mathfrak{C}} \leq n\left(\begin{array}{l}
n \\
n-1
\end{array}\right) \frac{2^{1 / 2}\left(S_{6} C_{6}\right)^{3} K_{l}}{4 \pi} C_{R}\left(4 \pi C_{\infty}\right)^{l-2}\|Q\|_{\mathcal{Q}}\left\|\rho^{\prime}\right\|_{\mathfrak{C}}^{l}
$$

- $\rho_{0, l}$ with $l \geq 5$. We want to estimate

$$
\begin{aligned}
& \left|\widehat{Q_{\zeta}}(p, p)\right| \leq \frac{1}{(2 \pi)^{1+\frac{3(n+1)}{2}}} \int_{-\infty}^{+\infty} d \eta \int \cdots \int \frac{1}{\left(E(p)^{2}+\eta^{2}\right)^{1 / 4}}\left|\widehat{\varphi^{\prime}}\left(p-p_{1}\right)\right| \times \\
\times \frac{1}{\left(E\left(p_{1}\right)^{2}+\eta^{2}\right)^{1 / 4}} & \prod_{j=1}^{n-1}\left(\frac{1}{\left(E\left(p_{j}\right)^{2}+\eta^{2}\right)^{1 / 4}}\left|\widehat{\varphi^{\prime}}\left(p_{j}-p_{j+1}\right)\right| \frac{1}{\left(E\left(p_{j+1}\right)^{2}+\eta^{2}\right)^{1 / 4}}\right) \times \\
& \times \frac{1}{\left(E\left(p_{n}\right)^{2}+\eta^{2}\right)^{1 / 4}}\left|\widehat{\zeta}\left(p_{n}-p\right)\right| \frac{1}{\left(E(p)^{2}+\eta^{2}\right)^{1 / 4}} d p_{1} \cdots d p_{n} .
\end{aligned}
$$

Since there are at least 6 functions, we may use (61) twice and obtain

$$
\left|\left\langle\rho_{0, l}^{\prime}, \zeta\right\rangle\right| \leq n \frac{\left(S_{6} C_{6}\right)^{6}}{4 \pi}\left(4 \pi C_{\infty}\right)^{l-5}\left(\frac{1}{2 \pi} \int_{-\infty}^{\infty} \frac{d \eta}{E(\eta)^{l-2}}\right)\left\|\rho^{\prime}\right\|_{\mathfrak{C}^{n}}^{n}\|\zeta\|_{\mathfrak{C}^{\prime}}
$$

and so

$$
\left\|\rho_{0, l}\right\|_{\mathfrak{C}} \leq n \frac{\left(S_{6} C_{6}\right)^{6}}{4 \pi}\left(4 \pi C_{\infty}\right)^{l-5} K_{l-2}\left\|\rho^{\prime}\right\|_{\mathfrak{C}^{\mathrm{c}}}^{n}
$$

Now since $K_{n-2} \leq K_{(n+1) / 2}$ when $n \geq 5$, we obtain

$$
\left\|\rho_{n}\right\|_{\mathfrak{C}} \leq n K_{\frac{n+1}{2}} C_{\rho}\left(C_{R} \sqrt{2}\|Q\|_{\mathcal{Q}}+2 \sqrt{\pi}\left\|\rho^{\prime}\right\|_{\mathfrak{C}}\right)^{n}
$$

with

$$
C_{\rho}:=\frac{S_{6} C_{6}}{4 \pi} \max \left(1,\left(\frac{C_{6} S_{6}}{2 \sqrt{\pi}}\right)^{2},\left(\frac{C_{6} S_{6}}{2 \sqrt{\pi}}\right)^{5}\right)=\frac{S_{6} C_{6}}{4 \pi}\left(\frac{C_{6} S_{6}}{2 \sqrt{\pi}}\right)^{5} .
$$

For $\rho_{4}$, we notice that $\rho_{0,4}=0$ for the same reason as $\rho_{0,2}$, and that $K_{2} \geq$ $K_{5 / 2}$. Therefore we obtain

$$
\left\|\rho_{4}\right\|_{\mathfrak{C}} \leq C_{\rho_{4}}\left(C_{R} \sqrt{2}\|Q\|_{\mathcal{Q}}+2 \sqrt{\pi}\left\|\rho^{\prime}\right\|_{\mathfrak{C}}\right)^{4}
$$


with

$$
C_{\rho_{4}}:=\frac{4 K_{2} S_{6} C_{6}}{4 \pi} \max \left(1,\left(\frac{C_{6} S_{6}}{2 \sqrt{\pi}}\right)^{2}\right)=\frac{S_{6} C_{6} K_{2}}{\pi}\left(\frac{C_{6} S_{6}}{2 \sqrt{\pi}}\right)^{2} .
$$

\subsubsection{The third order density $\rho_{3}$}

Lemma 16. We have

$$
\left\|\rho_{3}\right\|_{\mathfrak{C}} \leq C_{\rho_{3}}\left(C_{R} \sqrt{2}\|Q\|_{\mathcal{Q}}+2 \sqrt{\pi}\left\|\rho^{\prime}\right\|_{\mathfrak{C}}\right)^{3}
$$

and therefore

$$
\left\|\left(Q_{3}, \rho_{3}\right)\right\| \leq \kappa_{3}\left\|\left(Q, \rho^{\prime}\right)\right\|^{3}
$$

with

$$
\kappa_{3}=3 C_{R} K_{3 / 2} C_{Q} \sqrt{2}+2 \sqrt{\pi} C_{\rho_{3}}, \quad C_{\rho_{3}}=\frac{15 C_{M} S_{6}\left(S_{6,4}\right)^{2}\left(C_{6}\right)^{4}}{\pi\left(4 \pi C_{\infty}\right)^{3}} .
$$

Proof. Notice that thanks to the previous proof, we already have some estimates on $\rho_{3,0}, \rho_{2,1}$ and $\rho_{1,2}$. It remains to study $\rho_{0,3}$. As before and as in [29], we have to compute $\rho_{0,3}$ explicitely by a residuum formula. We thus write

$$
\rho_{0,3}=\sum_{\epsilon_{1}, \ldots, \epsilon_{4} \in\{ \pm\}} \rho_{0,3}^{\epsilon_{1} \epsilon_{2} \epsilon_{3} \epsilon_{4}}
$$

with an obvious definition.

- Let us treat first $\rho_{0,3}^{+---}$. We thus fix some $\zeta \in \mathfrak{C}^{\prime} \cap L^{2}$ and estimate the term

$$
\begin{aligned}
\widehat{Q_{\zeta}}(p, p)=(2 \pi)^{-6} \iiint d p_{1} d p_{2} d p_{3} \frac{\Lambda^{+}(p) \widehat{\varphi^{\prime}}\left(p-p_{1}\right) \Lambda^{-}\left(p_{1}\right)}{E(p)+E\left(p_{1}\right)} \times \\
\times \frac{\widehat{\varphi^{\prime}}\left(p_{1}-p_{2}\right) \Lambda^{-}\left(p_{2}\right) \widehat{\varphi^{\prime}}\left(p_{2}-p_{3}\right) \Lambda^{-}\left(p_{3}\right)}{\left(E(p)+E\left(p_{2}\right)\right)\left(E(p)+E\left(p_{3}\right)\right)} \widehat{\zeta}\left(p_{3}-p\right),
\end{aligned}
$$

by

$$
\begin{array}{rl}
\left|\widehat{Q_{\zeta}}(p, p)\right| \leq(2 \pi)^{-6} \iiint d p_{1} d p_{2} & d p_{3} \frac{\left|\Lambda^{+}(p) \widehat{\varphi^{\prime}}\left(p-p_{1}\right) \Lambda^{-}\left(p_{1}\right)\right|}{E\left(p+p_{1}\right)^{2 / 3}} \times \\
& \times \frac{\left|\widehat{\varphi^{\prime}}\left(p_{1}-p_{2}\right)\right|\left|\widehat{\varphi^{\prime}}\left(p_{2}-p_{3}\right)\right|}{E\left(p_{1}\right)^{1 / 3} E\left(p_{2}\right) E(p)}\left|\widehat{\zeta}\left(p_{3}-p\right)\right| .
\end{array}
$$

So if we follow the method used above, we obtain

$$
\left\|\rho_{0,3}^{+---}\right\| \mathfrak{C} \leq \frac{3 C_{M} S_{6}\left(S_{6,4}\right)^{2}\left(C_{6}\right)^{4}}{4 \pi}\left\|\rho^{\prime}\right\|_{\mathfrak{C}}^{3}
$$

Now, it is easily seen that $\rho_{0,3}^{-+++}, \rho_{0,3}^{--+}, \rho_{0,3}^{+++-}, \rho_{0,3}^{-+--}, \rho_{0,3}^{+-++}, \rho_{0,3}^{--+-}$ and $\rho_{0,3}^{++-+}$can be treated by exactly the same method. 
- Let us now treat for instance $\rho_{0,3}^{++--}$. Thanks to the residuum formula, we have to study

$$
\begin{aligned}
& \widehat{Q_{\zeta}}(p, p)=(2 \pi)^{-6} \iiint d p_{1} d p_{2} d p_{3} \Lambda^{+}(p) \widehat{\varphi^{\prime}}\left(p-p_{1}\right) \Lambda^{+}\left(p_{1}\right) \widehat{\varphi^{\prime}}\left(p_{1}-p_{2}\right) \Lambda^{-}\left(p_{2}\right) \\
& \widehat{\varphi^{\prime}}\left(p_{2}-p_{3}\right) \Lambda^{-}\left(p_{3}\right) \widehat{\zeta}\left(p_{3}-p\right) \times\left(\frac{1}{\left(E(p)+E\left(p_{2}\right)\right)\left(E\left(p_{1}\right)+E\left(p_{2}\right)\right)\left(E\left(p_{1}\right)+E\left(p_{3}\right)\right)}+\right. \\
&\left.\frac{1}{\left(E(p)+E\left(p_{2}\right)\right)\left(E(p)+E\left(p_{3}\right)\right)\left(E\left(p_{1}\right)+E\left(p_{3}\right)\right)}\right) .
\end{aligned}
$$

If we now use the same method as above for each of the two terms of this sum, we arrive at

$$
\left\|\rho_{0,3}^{++--}\right\|_{\mathfrak{C}} \leq 2 \frac{3 C_{M} S_{6}\left(S_{6,4}\right)^{2}\left(C_{6}\right)^{4}}{4 \pi}\left\|\rho^{\prime}\right\|_{\mathfrak{C}}^{3}
$$

This is easily generalized to the study of $\rho_{0,3}^{--+}, \rho_{0,3}^{+-+-}, \rho_{0,3}^{-+-+}, \rho_{0,3}^{+--+}$and $\rho_{0,3}^{-++-}$.

Summing now all these terms, we obtain

$$
\left\|\rho_{0,3}\right\|_{\mathfrak{C}} \leq 20 \frac{3 C_{M} S_{6}\left(S_{6,4}\right)^{2}\left(C_{6}\right)^{4}}{4 \pi}\left\|\rho^{\prime}\right\|_{\mathfrak{C}}^{3}=\frac{15 C_{M} S_{6}\left(S_{6,4}\right)^{2}\left(C_{6}\right)^{4}}{\pi}\left\|\rho^{\prime}\right\|_{\mathfrak{C}}^{3}
$$

and

$$
\left\|\rho_{3}\right\|_{\mathfrak{C}} \leq C_{\rho_{3}}\left(C_{R} \sqrt{2}\|Q\|_{\mathcal{Q}}+2 \sqrt{\pi}\left\|\rho^{\prime}\right\|_{\mathfrak{C}}\right)^{3}
$$

with

$$
\begin{aligned}
C_{\rho_{3}} & =3 \frac{S_{6} C_{6}}{4 \pi} \max \left(K_{3+1 / 2}, K_{3}, K_{2}\left(\frac{S_{6} C_{6}}{4 \pi C_{\infty}}\right)^{2}, \frac{20 C_{M}\left(S_{6,4}\right)^{2}\left(C_{6}\right)^{3}}{\left(4 \pi C_{\infty}\right)^{3}}\right) \\
& =\frac{15 C_{M} S_{6}\left(S_{6,4}\right)^{2}\left(C_{6}\right)^{4}}{\pi\left(4 \pi C_{\infty}\right)^{3}} .
\end{aligned}
$$

\subsubsection{List of constants}

We have used many constants in this proof. A summary which should help the reader to follow our arguments is provided in Table 1.

\section{Appendix: Derivation of the BDF energy}

In this section, we recall some basics about the second-quantization in nophoton $\mathrm{QED}$ and explain how the BDF energy $\mathcal{E}$ is derived from this theory, as a mean-field approximation. We mainly follow the method of Chaix-Iracane 9. 11], but with the notation of [54, 4, 30]. See also [29] for more details concerning the polarization of the vacuum. To simplify the presentation, we introduce $P_{-}^{0}:=P^{0}$ and $P_{+}^{0}:=1-P^{0}$. 


\begin{tabular}{|l|l|}
\hline Constant & defined in \\
\hline$C_{6}, C_{\infty}$ & Lemma [3] \\
$C_{R}$ & Equation (34) in Lemma 8 \\
$\kappa_{1}(\Lambda)$ & Equation (52) in Proposition 10] \\
$S_{p, q}, S_{p}, K_{p}$ & Equation (56) \\
$\kappa_{2}, C_{Q_{2}}, C_{\rho_{2}}$ & Lemma 13] \\
$C_{M}$ & Lemma [1] \\
$C_{Q}, C_{\rho}, C_{\rho_{4}}$ & Lemma [15] \\
$\kappa_{n}, n \geq 4$ & Lemma 15] \\
$C_{\rho_{3}}, \kappa_{3}$ & Lemma 16] \\
\hline
\end{tabular}

Table 1: Constants used in the proof of Theorem 3

\section{Free particles, Fock space, free vacuum}

We first introduce $\mathcal{F}_{+}^{(1)}:=P_{+}^{0} \mathcal{H}_{\Lambda}$ and $\mathcal{F}_{-}^{(1)}:=C P_{-}^{0} \mathcal{H}_{\Lambda}$ which are called respectively the free electron and the free positron state subspace. $C$ is the chargeconjugation operator defined by $C \psi=i \beta \alpha_{2} \bar{\psi}$. We define $\mathcal{F}_{+}^{(0)}=\mathcal{F}_{-}^{(0)}=\mathbb{C}$ and $\mathcal{F}_{+}^{(n)}=\bigwedge_{k=1}^{n} \mathcal{F}_{+}^{(1)}, \mathcal{F}_{-}^{(m)}=\bigwedge_{k=1}^{m} \mathcal{F}_{-}^{(1)}$ for $n, m \geq 1$. The space of $n$ free electrons and $m$ free positrons is then defined by $\mathcal{F}^{(n, m)}=\mathcal{F}_{+}^{(n)} \otimes \mathcal{F}_{-}^{(m)}$ and the associated Fock space is

$$
\mathcal{F}:=\bigoplus_{n, m=0}^{\infty} \mathcal{F}^{(n, m)} .
$$

For any $f \in \mathcal{H}_{\Lambda}$, the free electron (resp. positron) annihilation and creation operators $a_{0}(f)$ and $a_{0}^{*}(f)$ (resp. $b_{0}(f)$ and $\left.b_{0}^{*}(f)\right)$ are defined as usually [54. They fulfill the Canonical Anti-commutation Relations

$$
\begin{gathered}
\left\{a_{0}(f), a_{0}(g)\right\}=\left\{a_{0}(f), b_{0}(g)\right\}=\left\{b_{0}(f), b_{0}(g)\right\}=0, \\
\left\{a_{0}(f), a_{0}^{*}(g)\right\}=\left\langle f, P_{+}^{0} g\right\rangle, \quad\left\{b_{0}^{*}(f), b_{0}(g)\right\}=\left\langle f, P_{-}^{0} g\right\rangle,
\end{gathered}
$$

where $\langle\cdot, \cdot\rangle$ denotes the usual scalar product of $L^{2}\left(\mathbb{R}^{3}, \mathbb{C}^{4}\right)$.

The free vacuum state $\Omega_{0}$, a unit vector spanning $\mathcal{F}^{(0,0)}=\mathbb{C}$, is uniquely characterized up to a phase factor by the properties

$$
\left\|\Omega_{0}\right\|_{\mathcal{F}}=1, \quad a_{0}(f) \Omega_{0}=0 \quad \text { and } \quad b_{0}(f) \Omega_{0}=0,
$$

for all $f \in \mathcal{H}_{\Lambda}$.

The field operator $\Psi(f)$ is defined on the Fock space $\mathcal{F}$ by

$$
\Psi(f)=a_{0}(f)+b_{0}^{*}(f) .
$$

In terms of $\Psi(f)$, the CAR become, for all $(f, g) \in \mathcal{H}_{\Lambda}^{2}$,

$$
\{\Psi(f), \Psi(g)\}=\left\{\Psi^{*}(f), \Psi^{*}(g)\right\}=0, \quad\left\{\Psi(f), \Psi^{*}(g)\right\}=\langle f, g\rangle 1,
$$




\section{Dressed particles and vacuum}

In this description, the free electrons and positrons are defined with respect to the projector $P^{0}$, or equivalently the splitting $\mathcal{H}_{\Lambda}=\mathcal{H}_{-}^{0} \oplus \mathcal{H}_{+}^{0}$. We want now to change this definition and introduce the dressed electrons and positrons. To this end, we fix a new projector $P$ on $\mathcal{H}_{\Lambda}$, use again the notation $P_{-}:=P$ and $P_{+}:=1-P$, and introduce the dressed particle annihilation operators

$$
a_{P}(f):=\Psi\left(P_{+} f\right), \quad b_{P}(f):=\Psi^{*}\left(P_{-} f\right) .
$$

Similar formula can be given for the dressed particle creation operators $a_{P}^{*}$ and $b_{P}^{*}$. These dressed operators satisfy the same CAR as for the free operators (67) (68). We also introduce the dressed electrons and positrons state subspaces

$$
\mathcal{H}_{+}^{P}=(1-P) \mathcal{H}_{\Lambda}, \quad \mathcal{H}_{-}^{P}=P \mathcal{H}_{\Lambda} .
$$

Now, the main question is to know if there exists a dressed vacuum $\Omega_{P}$ in the Fock space $\mathcal{F}$. This state has to be a solution to the analogue of (69)

$$
\left\|\Omega_{P}\right\|_{\mathcal{F}}=1, \quad a_{P}(f) \Omega_{P}=0 \quad \text { and } \quad b_{P}(f) \Omega_{P}=0
$$

for all $f \in \mathcal{H}_{\Lambda}$. The answer is given by the celebrated

Theorem 4 (Shale-Stinespring Theorem [52]). There exists a dressed vacuum $\Omega_{P}$ in the Fock space $\mathcal{F}$ satisfying (71) if and only if $P-P^{0}$ is a HilbertSchmidt operator. In this case, $\Omega_{P}$ is unique up to a phase factor.

There are many proofs of this Theorem in the literature, see, e.g., [54, 36, 47]. and the references in [20]. This result explains why we assumed in the previous section that $P-P^{0} \in \mathfrak{S}_{2}\left(\mathcal{H}_{\Lambda}\right)$. Notice that $\Omega_{P}$ can be expressed as a rotation of the bare vacuum in the Fock space, $\Omega_{P}=\mathbb{U} \Omega_{0}$, $\mathbb{U}$ being called a Bogoliubov transformation. An explicit formula for $\Omega_{P}$ can be found in a lot of papers [54, 36, 47, 49, 50, 20].

The charge of the dressed vacuum $\Omega_{P}$ can be easily computed ${ }^{2}$

$$
\left\langle\Omega_{P}, \mathcal{Q} \Omega_{P}\right\rangle=\operatorname{tr}\left[P_{+}^{0}\left(P-P^{0}\right) P_{+}^{0}\right]+\operatorname{tr}\left[P_{-}^{0}\left(P-P^{0}\right) P_{-}^{0}\right]=\operatorname{tr}_{P^{0}}\left(P-P^{0}\right),
$$

justifying our study of Section 2.1. Here $\mathcal{Q}$ is the charge operator defined on $\mathcal{F}$ by [54, Formula (10.52)]

$\mathcal{Q}=\sum_{i \in \mathbb{Z} \backslash\{0\}}\left\{a_{0}^{*}\left(f_{i}\right) a_{0}\left(f_{i}\right)-b_{0}^{*}\left(f_{i}\right) b_{0}\left(f_{i}\right)\right\}=\sum_{i \geq 1} a_{0}^{*}\left(f_{i}\right) a_{0}\left(f_{i}\right)-\sum_{i \leq-1} b_{0}^{*}\left(f_{i}\right) b_{0}\left(f_{i}\right)$,

where $\left(f_{i}\right)_{i \geq 1}$ is an orthonormal basis of $\mathcal{H}_{+}^{0}$ and $\left(f_{i}\right)_{i \leq-1}$ is an orthonormal basis of $\mathcal{H}_{-}^{0}$.

\footnotetext{
${ }^{2}$ Notice that the charge of the dressed vacuum can also be easily obtained by using the explicit formula of $\Omega_{P}$, which immediately shows that it is an integer [54 37 50] 49].
} 


\section{Second-quantized Hamiltonian}

In the physics literature, the creation and annihilation operators are defined differently. For instance, instead of $a_{0}^{*}(f)$ which creates an electron in the state $P_{+}^{0} f$, the operator $a_{0}^{*}(x)$ which creates an electron at $x$ is formally used, where $a_{0}^{*}(x)=\sum_{i=1}^{\infty} a_{0}^{*}\left(f_{i}\right) f_{i}(x),\left(f_{i}\right)_{i \geq 1}$ being an orthonormal basis of $\mathcal{H}_{+}^{0}$. The operators $a_{P}(x)$ and $b_{P}(x)$ are defined similarly. We shall now use this formalism which is also the one of [29]. Formally, the CAR (67/68) are equivalent to

$$
\begin{gathered}
\left\{a_{0}(x), a_{0}(y)\right\}=\left\{a_{0}(x), b_{0}(y)\right\}=\left\{a_{0}^{*}(x), b_{0}(y)\right\}=\left\{b_{0}(x), b_{0}(y)\right\}=0, \\
\left\{a_{0}(x), a_{0}^{*}(y)\right\}=P_{+}^{0}(x, y), \quad\left\{b_{0}^{*}(x), b_{0}(y)\right\}=P^{0}(x, y) .
\end{gathered}
$$

We now start with writing down the formal unregularized no-photon Hamiltonian

$$
\mathbb{H}_{\mathrm{ur}}=\int d x \Psi^{*}(x) D^{\alpha \varphi} \Psi(x)+\frac{\alpha}{2} \int d x \int d y \frac{\Psi^{*}(x) \Psi(x) \Psi^{*}(y) \Psi(y)}{|\mathbf{x}-\mathbf{y}|},
$$

which acts on the Fock space $\mathcal{F}$. As explained for instance in [54, the free vacuum may not belong to the domain of this formally defined operator. Therefore, the expression (74) is renormalized by using a procedure which is called "normal ordering", denoted by double dots $:-:_{P^{0}}$. In each product of annihilation and creation operators, the $a_{0}^{*}$ and $b_{0}^{*}$ are moved to the left as if they anticommute with the $a_{0}$ and $b_{0}$. For instance

$$
\begin{aligned}
: \Psi^{*}(x) \Psi(y): P^{0} & =a_{0}^{*}(x) a_{0}(y)+a_{0}^{*}(y) b_{0}(x)+b_{0}(x) a_{0}(y)-b_{0}^{*}(y) b_{0}(x) \\
& =\Psi^{*}(x) \Psi(y)-P^{0}(x, y) .
\end{aligned}
$$

As a first step we thus regularize $\mathbb{H}_{\text {ur }}$ as done in Chaix and Iracane 9, Sections 3.5 and 4.1], namely we normal order with respect to the free projector $P^{0}$

$$
\mathbb{H}=\int d x: \Psi^{*}(x) D^{\alpha \varphi} \Psi(x):_{P^{0}}+\frac{\alpha}{2} \int d x \int d y \frac{: \Psi^{*}(x) \Psi(x) \Psi^{*}(y) \Psi(y):_{P^{0}}}{|\mathbf{x}-\mathbf{y}|} .
$$

This kind of regularization, which follows ideas of Dirac 13, 14, is standard in QED. It corresponds to the subtraction of the energy of the free Dirac sea, and the interaction energy with the free Dirac sea. A physical justification is given in [29, Section 3], on the basis of two guiding principles formulated by Weisskopf in [56]. The same choice is made in other studies dealing with vacuum polarization, for instance [9, 11, 36, 37, 49, 50, 20, 1. However, a better choice might be possible (see the paper [39] by Lieb and Siedentop, who propose another translation-invariant reference for normal ordering, in the absence of external field).

Now we can express $\mathbb{H}$ in terms of $:-:_{P}$ for some other $P$. Using (175) we obtain the reordering relations

$$
: \Psi^{*}(x) \Psi(y): P^{0}=: \Psi^{*}(x) \Psi(y):_{P}+Q(x, y)
$$


and

$$
\begin{aligned}
: \Psi^{*}(x) \Psi(x) \Psi^{*}(y) \Psi(y)::_{P^{0}}=: & \Psi^{*}(x) \Psi(x) \Psi^{*}(y) \Psi(y):_{P} \\
+2: \Psi^{*}(x) \Psi(x)::_{P} \operatorname{Tr}_{\mathbb{C}^{4}} & (Q(y, y))-2: \Psi^{*}(x) \Psi(y):_{P} Q(x, y) \\
& +\operatorname{Tr}_{\mathbb{C}^{4}}(Q(x, x)) \operatorname{Tr}_{\mathbb{C}^{4}}(Q(y, y))-|Q(x, y)|^{2}
\end{aligned}
$$

where $Q=P-P^{0}$. Therefore we can rewrite $\mathbb{H}$ with respect to an arbitrary dressed vacuum $P$ [9, formula (4.3)],

$$
\begin{gathered}
\mathbb{H}=\int: \Psi^{*}(x) D^{\alpha \varphi} \Psi(x):_{P} d x+\frac{\alpha}{2} \iint \frac{: \Psi^{*}(x) \Psi(x) \Psi^{*}(y) \Psi(y):_{P}}{|x-y|} d x d y \\
+\alpha \iint \frac{: \Psi^{*}(x) \Psi(x):_{P} \operatorname{Tr}_{\mathbb{C}^{4}}(Q(y, y))}{|x-y|} d x d y-\alpha \iint \frac{: \Psi^{*}(x) \Psi(y):_{P} Q(x, y)}{|x-y|} d x d y \\
+\operatorname{tr}_{P^{0}}\left(D^{\varphi} Q\right)+\frac{\alpha}{2} \iint \frac{\operatorname{Tr}_{\mathbb{C}^{4}}(Q(x, x)) \operatorname{Tr}_{\mathbb{C}^{4}}(Q(y, y))}{|x-y|} d x d y-\frac{\alpha}{2} \iint \frac{|Q(x, y)|^{2}}{|x-y|} d x d y .
\end{gathered}
$$

The last line represents the energy of the dressed vacuum $P$ measured with respect to $P^{0}$, whereas in the second line the vacuum polarization potentials appear.

\section{Restriction to Bogoliubov-Dirac-Fock states}

We now follow [9] and restrict ourselves to Bogoliubov-Dirac-Fock type states. In this approximation method, a dressed vacuum $P$ is first chosen such that $P-P^{0} \in \mathfrak{S}_{2}(\mathcal{H})$. Then a $B D F$ state is simply a Slater determinant made with $n$ electrons and $m$ positrons defined with respect to the dressed vacuum $P(n, m \geq 0$ are not fixed in this theory). This is a state of $\mathcal{F}$ which takes the form

$$
\psi=a_{P}^{*}\left(f_{1}\right) \cdots a_{P}^{*}\left(f_{n}\right) b_{P}^{*}\left(g_{1}\right) \cdots b_{P}^{*}\left(g_{m}\right) \Omega_{P},
$$

where $\left(f_{1}, \ldots, f_{n}\right) \in\left(\mathcal{H}_{+}^{P}\right)^{n}$ and $\left(g_{1}, \ldots, g_{m}\right) \in\left(\mathcal{H}_{-}^{P}\right)^{m}$ are such that $\left\langle f_{i}, f_{j}\right\rangle=\delta_{i j}$, $\left\langle g_{i}, g_{j}\right\rangle=\delta_{i j}$, and $\Omega_{P}$ is the dressed vacuum in $\mathcal{F}$ obtained by Theorem 4 Since it is easily seen that $\psi=\Omega_{P^{\prime}}$ where

$$
P^{\prime}=P+\gamma, \quad \gamma=\sum_{i=1}^{n}\left|f_{i}\right\rangle\left\langle f_{i}\left|-\sum_{j=1}^{m}\right| g_{j}\right\rangle\left\langle g_{j}\right|,
$$

we obtain immediately from (78)

$$
\langle\psi|\mathbb{H}| \psi\rangle=\left\langle\Omega_{P^{\prime}}|\mathbb{H}| \Omega_{P^{\prime}}\right\rangle=\mathcal{E}\left(P^{\prime}-P^{0}\right)=\mathcal{E}\left(P-P^{0}+\gamma\right) .
$$

In [9] formula (4.8)], this formula is expanded like in (12) in terms of the vacuum density matrix $Q=P-P^{0}$ and the density $\gamma$ of the dressed particles. 


\section{References}

[1] A. Aste, G. Baur, K. Hencken, D. Trautmann, G. Scharf. Electron-positron pair production in the external electromagnetic field of colliding relativistic heavy ions. Eur. Phys. J. C, 23(3), 545-550, 2002.

[2] J. Avron, R. Seiler, B. Simon. The Index of a Pair of Projections. Journal of Functional Analysis, 120: 220-237, 1994.

[3] V. Bach. Error bound for the Hartree-Fock energy of atoms and molecules. Comm. Math. Phys., 147: 527-548, 1992.

[4] V. Bach, J.-M. Barbaroux, B. Helffer, H. Siedentop. On the Stability of the Relativistic Electron-Positron Field. Comm. Math. Phys., 201: 445460, 1999 .

[5] V. Bach, E.H. Lieb, J.P. Solovej. Generalized Hartree-Fock theory and the Hubbard model. J. Statist. Phys., 76 (1-2): 3-89, 1994.

[6] J.M. Barbaroux, M.J. Esteban, E. Séré. Some connections between DiracFock and Electron-Positron Hartree-Fock. Ann. Henri Poincaré, 6(1): 85$102,2005$.

[7] J.-M. Barbaroux, W. Farkas, B. Helffer, H. Siedentop. On the Hartree-Fock equations of the electron/positron field. To appear in Comm. Math. Phys.

[8] E. Cancès. SCF algorithms for HF electronic calculations. Defranceschi, M. (ed.) et al., Mathematical models and methods for ab initio quantum chemistry. Berlin: Springer. Lect. Notes Chem. 74: 17-43, 2000.

[9] P. Chaix, D. Iracane. From quantum electrodynamics to mean field theory: I. The Bogoliubov-Dirac-Fock formalism. J. Phys. B, 22(23): 3791-3814, 1989.

[10] P. Chaix, D. Iracane, P.L. Lions. From quantum electrodynamics to mean field theory: II. Variational stability of the vacuum of quantum electrodynamics in the mean-field approximation. J. Phys. B, 22(23): 3815-3828, 1989.

[11] P. Chaix. Une Méthode de Champ Moyen Relativiste et Application à l'Etude du Vide de l'Electrodynamique Quantique. PhD Thesis, University Paris VI, 1990.

[12] J.P. Desclaux. Relativistic Dirac-Fock expectation values for atoms with $Z=1$ to $Z=120$. Atomic Data and Nuclear Data Tables 12 (1973), p. 311-406.

[13] P.A.M. Dirac. Théorie du positron. Solvay report, pages 203-212. Paris: Gauthier-Villars. XXV, 353 S., 1934.

[14] P.A.M. Dirac. Discussion of the infinite distribution of electrons in the theory of the positron. Proc. Camb. Philos. Soc., 30: 150-163, 1934. 
[15] M.J. Esteban, E. Séré. Solutions of the Dirac-Fock Equations for Atoms and Molecules. Comm. Math. Phys., 203: 499-530, 1999.

[16] M.J. Esteban, E. Séré. Nonrelativistic limit of the Dirac-Fock equations. Ann. Henri Poincaré, 2(5): 941-961, 2001.

[17] M.J. Esteban, E. Séré. A max-min principle for the ground state of the Dirac-Fock functional. Contemp. Math., 307: 135-139, 2002.

[18] L.L. Foldy, E. Eriksen. Some Physical Consequences of Vacuum Polarization. Phys. Rev., 95(4): 1048-1051, 1954.

[19] J.D. French, V.F. Weisskopf. The Electromagnetic Shift of Energy Levels. Phys. Rev., II. Ser., 75: 1240-1248, 1949.

[20] H. Fierz, G. Scharf. Particle interpretation for external field problems in QED. Helv. Phys. Acta, 52: 437-453, 1979.

[21] W.H. Furry. On Bound States and Scattering in Positron Theory. Phys. Rev., 81(1): 115-124, 1951.

[22] W.H. Furry. A symmetry theorem in the positron theory. Phys. Rev., 51: 125-129, 1937.

[23] W.H. Furry, J.R. Oppenheimer. On the Theory of the Electron and Positive. Phys. Rev., II. Ser., 45: 245-262, 1934.

[24] R. Glauber, W. Rarita, P. Schwed. Vacuum Polarization Effects on Energy Levels in $\mu$-Mesonic Atoms. Phys. Rev., 120(2): 609-613, 1960.

[25] O. Gorceix, P. Indelicato, J.P. Desclaux. Multiconfiguration Dirac-Fock studies of two-electron ions: I. Electron-electron interaction. J. Phys. B: At. Mol. Phys. 20 (1987), p. 639-649.

[26] I.P. Grant. Relativistic Calculation of Atomic Structures. Adv. Phys. 19 (1970), p. 747-811.

[27] C. Hainzl. On the Vacuum Polarization Density caused by an External Field. Ann. Henri Poincaré, 5: 1137-1157, 2004.

[28] C. Hainzl, M. Lewin, E. Séré. Self-consistent solution for the polarized vacuum in a no-photon QED model. To appear in J. Phys. A: Math. and Gen.

[29] C. Hainzl, H. Siedentop. Non-Perturbative Mass and Charge Renormalization in Relativistic no-photon Quantum Electrodynamics. Comm. Math. Phys., 243: 241-260, 2003.

[30] B. Helffer, H. Siedentop. Form perturbation of the second quantized Dirac field. Mathematical Physics Electronic Journal, 4, paper 4, 1998.

[31] W. Heisenberg. Bemerkungen zur Diracschen Theorie des Positrons. Z. Phys., 90: 209-231, 1934. 
[32] D. Hundertmark, N. Röhrl, H. Siedentop. The sharp bound on the stability of the relativistic electron-positron field in Hartree-Fock approximation. Comm. Math. Phys., 211 (3): 629-642, 2000.

[33] C. Itzykson, J.-B. Zuber. Quantum Field Theory. McGraw-Hill, New York, 1980.

[34] Y.K. Kim. Relativistic self-consistent Field theory for closed-shell atoms. Phys. Rev. 154 (1967), p. 17-39.

[35] M. Klaus. Non-regularity of the Coulomb potential in quantum electrodynamics. Helv. Phys. Acta, 53: 36-39, 1980.

[36] M. Klaus, G. Scharf. The regular external field problem in quantum electrodynamics. Helv. Phys. Acta, 50: 779-802, 1977.

[37] M. Klaus, G. Scharf. Vacuum Polarization in Fock Space. Helv. Phys. Acta, 50: 803-814, 1977.

[38] E.H. Lieb. Variational Principle for Many-Fermion Systems. Phys. Rev. Lett., 46: 457-459, 1981.

[39] E.H. Lieb, H. Siedentop. Renormalization of the regularized relativistic electron-positron field. Comm. Math. Phys., 213 (3): 673-683, 2000.

[40] E.H. Lieb, B. Simon. The Hartree-Fock theory for Coulomb systems. Comm. Math. Phys., 53: 185-194, 1977.

[41] I. Lindgren, A. Rosen. Relativistic self-consistent field calculations. Case Stud. At. Phys. 4 (1974), p. 93-149.

[42] M.H. Mittleman. Theory of Relativistic effects on atoms: Configurationspace Hamiltonian. Phys. Rev. A, 24 (3): 1167-1175, 1981.

[43] P. J. Mohr, G. Plunien, G. Soff. QED corrections in Heavy Atoms. Phys. Rep, 293: 227-369, 1998.

[44] E. Paturel. Solutions of the Dirac-Fock equations without projector. Ann. Henri Poincaré, 1(6): 1123-1157, 2000.

[45] W. Pauli, M.E. Rose. Remarks on the Polarization Effects in the Positron Theory. Phys. Rev II, 49 : 462-465, 1936.

[46] J. Reinhardt, B. Müller, W. Greiner. Theory of positron production in heavy-ion collision. Phys. Rev. A, 24(1):103-128, 1981.

[47] S.N.M. Ruijsenaars. On Bogoliubov transformations for systems of relativistic charged particles. J. Math. Phys., 18(3): 517-526, 1977.

[48] B. Simon. Trace Ideals and their Applications. Vol 35 of London Mathematical Society Lecture Notes Series. Cambridge University Press, 1979. 
[49] G. Scharf, H.P. Seipp. Charged Vacuum, Spontaneous Positron Production and All That. Phys. Lett., 108B(3): 196-198, 1982.

[50] H.P. Seipp. On the $S$-operator for the external field problem of QED. Helv. Phys. Acta, 55: 1-28, 1982.

[51] R. Serber. Linear modifications in the Maxwell field equations. Phys. Rev., II. Ser., 48: 49-54, 1935.

[52] D. Shale, W.F. Stinespring. Spinor representations of infinite orthogonal groups. J. Math. and Mech., 14: 315-322, 1965.

[53] B. Swirles. The relativistic self-consistent field. Proc. Roy. Soc. A 152 (1935), p. 625-649.

[54] B. Thaller. The Dirac Equation. Springer Verlag, 1992.

[55] E.A. Uehling. Polarization effects in the positron theory. Phys. Rev., II. Ser., 48: 55-63, 1935.

[56] V. Weisskopf. Über die Elektrodynamik des Vakuums auf Grund der Quantentheorie des Elektrons. Math.-Fys. Medd., Danske Vid. Selsk., 16(6):139,1936 .

[57] J.H. Wolkowisky. Existence of solutions of the Hartree equations for $N$ electrons. An application of the Schauder-Tychonoff theorem. Indiana Univ. Math. J., 22: 551-568, 1972-73. 